\title{
Modelo GARCH com Mudança \\ de Regime Markoviano para séries financeiras
}

\author{
William Gonzalo Rojas Durán
}

\author{
DISSERTAÇÃO APRESENTADA \\ $\mathrm{AO}$ \\ Instituto DE MATEMÁticA E EstatísticA \\ DA \\ Universidade DE SÃo Paulo \\ PARA \\ OBTENÇÃO DO TÍTULO \\ DE \\ Mestre EM CiÊnCIAS \\ Programa: Estatística \\ Orientador: Prof. Dr. Airlane Pereira Alencar
}

Durante o desenvolvimento deste trabalho o autor recebeu auxílio financeiro da CNPq

São Paulo, Março de 2014 


\section{Modelo GARCH com Mudança de Regime Markoviano para séries financeiras}

Esta versão final da dissertação contém as correções e alterações sugeridas pela Comissão Julgadora durante a defesa da versão original do trabalho, realizada em 24/03/2014.

Comissão Julgadora:

- Prof $^{\mathrm{a}}$. Dr ${ }^{\mathrm{a}}$. Airlane Pereira Alencar (orientadora) - IME-USP

- Prof. Dr. Pedro Alberto Morettin - IME-USP

- Prof ${ }^{a}$. Dr ${ }^{\mathrm{a}}$. Thelma Sáfadi - UFLA 


\section{Agradecimentos}

Primeiramente, agradeço a minha familia que de alguma ou outra forma me ajudaram neste caminho.

Agradeço a LARC por seu apoio incondicional em todo momento.

Agradeço a Professora Airlane pela ajuda constante neste trabalho.

Aos Professores Pedro Morettin e Thelma Sáfadi pelas correções feitas no trabalho.

A meus amigos do IME, Elisângela, Josemir, Agatha, Ana Paula, Andressa, Alejandra, Fábio Oki.

Aos professores do IME. 


\section{Resumo}

ROJAS, D. W. G. Modelo GARCH com Mudança de Regime Markoviano para séries financeiras. 2013. 120 f. Dissertação (Mestrado) - Instituto de Matemática e Estatística, Universidade de São Paulo, São Paulo, 2013.

Neste trabalho analisaremos a utilização dos modelos de mudança de regime markoviano para a variância condicional. Estes modelos podem estimar de maneira fácil e inteligente a variância condicional não observada em função da variância anterior e do regime. Isso porque, é razoável ter coeficientes variando no tempo dependendo do regime correspondentes à persistência da variância (variância anterior) e às inovações. A noção de que uma série econômica possa ter alguma variação na sua estrutura é antiga para os economistas. Marcucci (2005) comparou diferentes modelos com e sem mudança de regime em termos de sua capacidade para descrever e predizer a volatilidade do mercado de valores dos EUA. O trabalho de Hamilton (1989) foi uns dos mais importantes para o desenvolvimento de modelos com mudança de regime. Inicialmente mostrou que a série do PIB dos EUA pode ser modelada como um processo que tem duas formas diferentes, uma na qual a economia encontra-se em crescimento e a outra durante a recessão. O câmbio de uma fase para outra da economia pode seguir uma cadeia de Markov de primeira ordem.

Utilizamos as séries de índice Bovespa e S\&P500 entre janeiro de 2003 e abril de 2012 e ajustamos o modelo GARCH $(1,1)$ com mudança de regime seguindo uma cadeia de Markov de primeira ordem, considerando dois regimes. Foram consideradas as distribuições gaussiana, $t$ de Student e generalizada do erro (GED) para modelar as inovações.

A distribuição $t$ de Student com mesmo grau de liberdade para ambos os regimes e graus distintos se mostrou superior à distribuição normal para caracterizar a distribuição dos retornos em relação ao modelo GARCH com mudança de regime. Além disso, verificou-se um ganho no percentual de cobertura dos intervalos de confiança para a distribuição normal, bem como para a distribuição $t$ de Student com mesmo grau de liberdade para ambos os regimes e graus distintos, em relação ao modelo GARCH com mudança de regime quando comparado ao modelo GARCH usual.

Palavras-chave: Mudança de Regime Markoviano, Volatilidade, modelos GARCH. 


\section{Abstract}

ROJAS, D. W. G. Markov Regime Switching GARCH Model for Financial Series. 2010. 120 f. Thesis (Master) - Mathematics and Statistics Institute, University of São Paulo, São Paulo, 2013.

In this work we analyze heterocedastic financial data using Markov regime switching models for conditional variance. These models can estimate easily the unobserved conditional variance as function of the previous variance and the regime. It is reasonable to have time-varying coefficients corresponding to the persistence of variance (previous variance) and innovations. The economic series notion may have some variation in their structure is usual for economists. Marcucci (2005) compared different models with and without regime switching in terms of their ability to describe and predict the volatility of the U.S. market. The Hamilton's (1989) work was the most important one in the regime switching models development. Initially showed that the series of U.S. GDP can be modeled as a process that has two different forms one in which the economy is growing and the other during the recession. The change from one phase to another economy can follow a Markov first order chain.

We use the Bovespa series index and S\&P500 between January 2003 and April 2012 and fitted the GARCH $(1,1)$ models with regime switching following a Markov first order chain, considering two regimes. We considered Gaussian distribution, Student-t and generalized error (GED) to model innovations.

The $t$-Student distribution with the same freedom degree for both regimes and distinct degrees showed higher than normal distribution for characterizing the distribution of returns relative to the GARCH model with regime switching. In addition, there was a gain in the percentage of coverage of the confidence intervals for the normal distribution, as well as the $t$-Student distribution with the same freedom degree for both regimes and distinct degrees related to GARCH model with regime switching when compared to the usual GARCH model.

Keywords: Markov Regime Switching, Volatility, GARCH models. 


\section{Sumário}

$\begin{array}{ll}\text { Lista de Figuras } & \text { ix }\end{array}$

Lista de Tabelas $\quad$ xiii

1 Introdução 1

2 Conceitos $\quad 3$

2.1 Séries Temporais Estacionárias e Ergódicas . . . . . . . . . . . . . . . . . 3

2.1 .1 Processos Lineares . . . . . . . . . . . . . . . . . . . . . 4

2.2 Séries Temporais Não Estacionárias . . . . . . . . . . . . . . . . . . 5

2.2 .1 Modelos da família ARIMA . . . . . . . . . . . . . . . . . 5

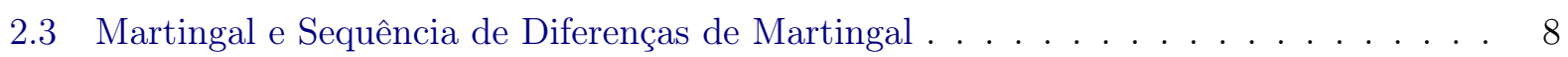

2.4 Variância de Longo Prazo . . . . . . . . . . . . . . . . . . . . . . . 8

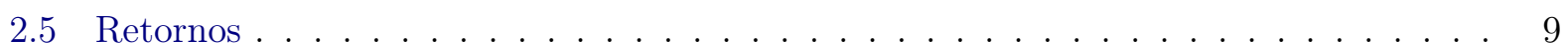

3 Modelos GARCH $\quad 11$

3.1 Introdução . . . . . . . . . . . . . . . . . . . . . . . . . . . . . 11

3.2 Modelos ARCH . . . . . . . . . . . . . . . . . . . . . . . 11

3.3 Testes para efeitos com Heteroscedasticidade Condicional . . . . . . . . . . . . . . 13

3.4 Modelo GARCH . . . . . . . . . . . . . . . . . . . . . . . . 14

3.5 Modelo GARCH e Fatos Estilizados . . . . . . . . . . . . . . . . . . . . 15

3.6 Estimação de Modelos GARCH . . . . . . . . . . . . . . . . . . . . . . 15

3.6.1 Estimador de Máxima Verossimilhança Condicional . . . . . . . . . . . . . . . 16

3.6.2 Estimadores de Mínimos Desvios Absolutos . . . . . . . . . . . . . . . . . . 17

3.7 Diagnóstico de Modelos GARCH . . . . . . . . . . . . . . . . . . . . 18

3.8 Extensões dos Modelos GARCH . . . . . . . . . . . . . . . . . . . . . . . 20

3.9 Distribuição de Erros Não-Gaussianos . . . . . . . . . . . . . . . . . . . . . . 21

3.10 Seleção e Comparação de Modelos GARCH . . . . . . . . . . . . . . . . . . . . . 22

3.11 Predição de Modelos GARCH . . . . . . . . . . . . . . . . . . . . . . 24

4 Modelo GARCH com Mudança de Regime $\quad 25$

4.1 Modelo GARCH com mudança de regime Markoviano . . . . . . . . . . . . . . . 25

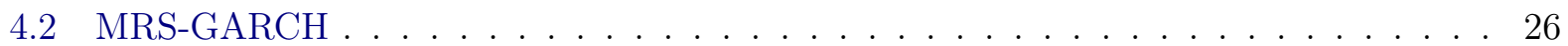

4.3 Estimação . . . . . . . . . . . . . . . . . . . . . . . . . . 27

4.4 Probabilidade Suavizada . . . . . . . . . . . . . . . . . . . . . . 28 
5 Aplicação em Séries Reais

5.1 Aplicações . . . . . . . . . . . . . . . . . . . . . . . . . . 31

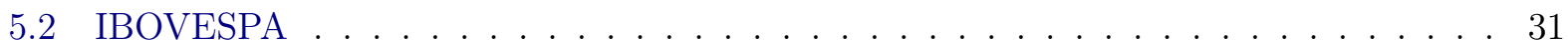

5.3 Análise de Resíduos-IBOVESPA . . . . . . . . . . . . . . . . . . . . 39

5.3.1 Análise de Resíduos para o modelo MRS-GARCH-N . . . . . . . . . . . . . . 40

5.3.2 Análise de Resíduos para o modelo MRS-GARCH-t2 . . . . . . . . . . . . . . 42

5.3.3 Análise de Resíduos para o modelo MRS-GARCH-t . . . . . . . . . . . . . . . 44

5.3.4 Análise de Resíduos para o modelo MRS-GARCH-GED . . . . . . . . . . . . 46

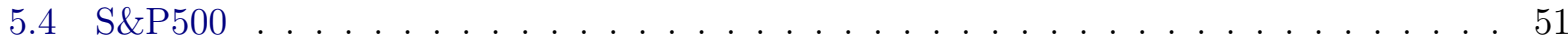

5.5 Análise de Resíduos-S\&P500 . . . . . . . . . . . . . . . . . . . . . . . . 58

5.5.1 Análise de Resíduos para o modelo MRS-GARCH-N . . . . . . . . . . . . . . 58

5.5.2 Análise de Resíduos para o modelo MRS-GARCH-t2 . . . . . . . . . . . . . . 60

5.5.3 Análise de Resíduos para o modelo MRS-GARCH-GED . . . . . . . . . . . . 62

$\begin{array}{llr}6 & \text { Conclusões } & 67\end{array}$

A Mistura de Distribuições $\quad 69$

Referências Bibliográficas $\quad 73$ 


\section{Lista de Figuras}

3.1 Gráfico da Série do índice Bovespa e os Log-retornos. . . . . . . . . . . . . . . . . . 12

3.2 Gráfico da função de autocorrelação e autocorrelação parcial da Série dos log-retornos do índice Bovespa . . . . . . . . . . . . . . . . . . . . . . . . . 12

3.3 Gráfico de quantis dos Resíduos padronizados do modelo $\operatorname{GARCH}(1,1)$ da série de retornos do Ibovespa . . . . . . . . . . . . . . . . . . . . . 20

3.4 Função de autocorrelação e função de autocorrelação ao quadrado dos resíduos padronizados - Modelo GARCH $(1,1)$ da série de retornos do Ibovespa . . . . . . . . . 20

3.5 Função de autocorrelação e função de autocorrelação ao quadrado dos resíduos padronizados da Distribuição Normal e t-Student . . . . . . . . . . . . . . . . . . 23

3.6 Gráfico de quantis dos Resíduos Padronizados da Distribuição Normal e t-Student 23

5.1 Gráfico da Série do índice Bovespa e os Log-retornos. . . . . . . . . . . . . . . . . . . 31

5.2 Gráfico de quantis e Histograma dos log-retornos da Série do índice Bovespa. .. . . 32

5.3 Função de autocorrelação dos log-retornos e log-retornos ao quadrado-IBOVESPA. . 33

5.4 Probabilidade suavizada do Regime 1 - Ibovespa - Normal. . . . . . . . . . . . . . . . 35

5.5 Estimativa do desvio padrão condicional para os retornos do Ibovespa no Regime 1 e 2 com erro gaussiano. . . . . . . . . . . . . . . . . . . . . 36

5.6 Probabilidade suavizada do Regime 1 - Ibovespa - t. . . . . . . . . . . . . 36

5.7 Estimativa do desvio padrão condicional para os retornos do Ibovespa no Regime 1 e 2 com erro t de Student. . . . . . . . . . . . . . . . . . . . . 37

5.8 Probabilidade suavizada do Regime 1 - Ibovespa - t2 . . . . . . . . . . . . . 37

5.9 Estimativa do desvio padrão condicional para os retornos do Ibovespa no Regime 1 e 2 com erro t de Student-t2 . . . . . . . . . . . . . . . . . . . . . 38

5.10 Probabilidade suavizada do Regime 1 - Ibovespa - GED . . . . . . . . . . . . . 38

5.11 Estimativa do desvio padrão condicional para os retornos do Ibovespa no Regime 1 e 2 com erro GED. . . . . . . . . . . . . . . . . . . . . . . . . . . . 39

5.12 Função de autocorrelação e função de autocorrelação ao quadrado dos resíduos padronizados do modelo Normal-Ibovespa. . . . . . . . . . . . . . . . . . . . 40

5.13 Resíduos padronizados nos Regimes 1 e 2 do modelo Normal Ibovespa. . . . . . . . . 40

5.14 Gráfico de quantis dos Resíduos padronizados do modelo Normal-Ibovespa. . . . . . 41

5.15 Histograma dos resíduos padronizados do modelo Normal-Ibovespa. . . . . . . . . . . 41

5.16 Função de autocorrelação e função de autocorrelação ao quadrado dos resíduos padronizados do modelo t2-Ibovespa. . . . . . . . . . . . . . . . . . . . 42

5.17 Resíduos padronizados nos Regimes 1 e 2 do modelo t2-Ibovespa. . . . . . . . . . . . 42 
5.18 Gráfico de quantis dos Resíduos padronizados do modelo t2-Ibovespa. . . . . . . . . . 43

5.19 Histograma dos Resíduos padronizados do modelo t2-Ibovespa. . . . . . . . . . . . . 43

5.20 Função de autocorrelação e função de autocorrelação ao quadrado dos resíduos padronizados do modelo t-Ibovespa. . . . . . . . . . . . . . . . . . . . . . . 44

5.21 Resíduos padronizados nos Regimes 1 e 2 do modelo t-Ibovespa. . . . . . . . . . . . . 44

5.22 Gráfico de quantis dos Resíduos padronizados do modelo t-Ibovespa. . . . . . . . . . 45

5.23 Histograma dos Resíduos padronizados do modelo t-Ibovespa. . . . . . . . . . . . . . 45

5.24 Função de autocorrelação e função de autocorrelação ao quadrado dos resíduos padronizados do modelo GED-Ibovespa. . . . . . . . . . . . . . . . . 46

5.25 Resíduos padronizados nos Regimes 1 e 2 do modelo GED-Ibovespa. . . . . . . . . . 46

5.26 Gráfico de quantis dos Resíduos padronizados do modelo GED-Ibovespa. . . . . . . . 47

5.27 Histograma dos Resíduos padronizados do modelo GED-Ibovespa. . . . . . . . . . . . 47

5.28 Retornos e Intervalos de confiança (95\%)-Normal-Ibovespa. . . . . . . . . . . . . . 48

5.29 Retornos e Intervalos de confiança $(95 \%)$-t-Ibovespa. . . . . . . . . . . . . . . . . 48

5.30 Retornos e Intervalos de confiança (95\%)-t2-Ibovespa. . . . . . . . . . . . . . . . 49

5.31 Retornos e Intervalos de confiança (95\%)-GED-Ibovespa. . . . . . . . . . . . . . . 49

5.32 Gráfico da Série S\&P500 e os Log-retornos. . . . . . . . . . . . . . . . . . . . 51

5.33 Gráfico de quantis e Histograma dos log-retornos da Série S\&P500. . . . . . . . . . . 51

5.34 Função de autocorrelação dos log-retornos e log-retornos ao quadrado-S\&P500. . . . 52

5.35 Probabilidade suavizada do Regime 1 - Normal - S\&P500. . . . . . . . . . . . . . . 55

5.36 Estimativa do desvio padrão condicional para os retornos da S\&P500 no Regime 1 e 2 com erro gaussiano. . . . . . . . . . . . . . . . . . . . 5 55

5.37 Probabilidade suavizada do Regime 1- t2 - S\&P500 . . . . . . . . . . . . . . 56

5.38 Estimativa do desvio padrão condicional para os retornos da S\&P500 no Regime 1 e 2 com erro t de Student. . . . . . . . . . . . . . . . . . . . . 56

5.39 Probabilidade suavizada do Regime 1 - GED - S\&P500. . . . . . . . . . . . . 57

5.40 Estimativa do desvio padrão condicional para os retornos da S\&P500 no Regime 1 e 2 com erro GED. . . . . . . . . . . . . . . . . . . . 5 57

5.41 Função de autocorrelação e função de autocorrelação ao quadrado dos resíduos padronizados do modelo Normal-S\&P500 . . . . . . . . . . . . . . . . . 58

5.42 Resíduos padronizados nos Regimes 1 e 2 do modelo Normal-S\&P500. . . . . . . . . 59

5.43 Gráfico de quantis dos Resíduos padronizados do modelo Normal-S\&P500. . . . . . . 59

5.44 Histograma dos Resíduos padronizados do modelo Normal-S\&P500 . . . . . . . . . . 59

5.45 Função de autocorrelação e função de autocorrelação ao quadrado dos resíduos padronizados do modelo t2-S\&P500. . . . . . . . . . . . . . . . . . . 60

5.46 Resíduos padronizados nos Regimes 1 e 2 do modelo t2-S\&P500. . . . . . . . . . . . 60

5.47 Gráfico de quantis dos Resíduos padronizados do modelo t2-S\&P500 . . . . . . . . . . 61

5.48 Histograma dos Resíduos padronizados do modelo t2-S\&P500 . . . . . . . . . . . . . 61

5.49 Função de autocorrelação e função de autocorrelação ao quadrado dos resíduos padronizados do modelo GED-S\&P500 . . . . . . . . . . . . . . . . . . 62

5.50 Resíduos padronizados nos Regimes 1 e 2 do modelo GED-S\&P500 . . . . . . . . . . 62

5.51 Gráfico de quantis dos Resíduos padronizados do modelo GED-S\&P500. . . . . . . . 63

5.52 Histograma dos Resíduos padronizados do modelo GED-S\&P500. . . . . . . . . . . . 63 
5.53 Retornos e Intervalos de confiança (95\%)-Normal-S\&P500. . . . . . . . . . . . . . . 64

5.54 Retornos e Intervalos de confiança $(95 \%)$-t2-S\&P500 . . . . . . . . . . . . . . 64

5.55 Retornos e Intervalos de confiança $(95 \%)$-GED-S\&P500. . . . . . . . . . . . . . 65 


\section{Lista de Tabelas}

3.1 Coeficientes Estimados do modelo $\operatorname{GARCH}(1,1) \ldots \ldots \ldots \ldots \ldots$

3.2 Testes para diagnóstico do modelo $\operatorname{GARCH}(1,1)$ ajustado $\ldots \ldots \ldots \ldots$

3.3 AIC, BIC e log da verossimilhança dos modelos $\operatorname{GARCH}(1,1)$ ajustados para a série de retornos Ibovespa . . . . . . . . . . . . . . . . . . . . . . 23

5.1 Medidas descritivas do retorno-Ibovespa . . . . . . . . . . . . . . . . . 32

5.2 Estimativas de Máxima Verossimilhança erros padrões do Modelo MRS - GARCH Ibovespa . . . . . . . . . . . . . . . . . . . . . . . 34

5.3 Porcentagem de Cobertura do intervalos de confiança para os log-retornos do Ibovespa 50

5.4 Medidas descritivas do retorno-S\&P500 . . . . . . . . . . . . . . . . . 52

5.5 Estimativas de Máxima Verossimilhança e erros padrões do Modelo MRS - GARCH $-\mathrm{S} \& \mathrm{P} 500 \ldots \ldots \ldots \ldots \ldots \ldots \ldots \ldots$

5.6 Porcentagem de Cobertura do intervalos de confiança para os log-retornos da S\&P500 65 


\section{Capítulo 1}

\section{Introdução}

Neste trabalho analisaremos a utilização dos modelos de mudança de regime markoviano para a variância condicional. Estes modelos podem estimar de maneira fácil e inteligente a variância condicional não observada em função da variância anterior e do regime. No entanto, é razoável ter coeficientes variando no tempo correspondentes à persistência da variância (variância anterior) e às inovações.

Séries temporais apresentam mudanças de comportamento ao longo do tempo que se caracterizam por diversos regimes. Um exemplo é quando o mercado se encontra em dois possíveis regimes: recessão e expansão do mercado. Esses regimes não são variáveis medidas diretamente e, portanto, constituem variáveis aleatórias. O trabalho de Hamilton (1989) foi uns dos mais importantes para o desenvolvimento de modelos com mudança de regime. Quandt (1972) apresenta um modelo de regressão com mudanças independentes de regime e Goldfeld e Quandt (1973), amplia a análise para modelos com mudanças de regimes markovianas.

A principal motivação deste trabalho é propor um modelo de volatilidade com mudança de regime markoviana, regime este associado aos parâmetros do modelo.

Adotamos o modelo MRS-GARCH introduzido por Klaassen (2002) usando as distribuições normal, $t$-Student e a distribuição generalizada do erro (GED). Além disso, o parâmetro $\nu$ (graus de libertade) se deixa variar entre diferentes regimes, para capturar a curtose. De uma forma geral, Dueker (1997) mostrou que no modelo com mudança de regime apenas alguns parâmetros são dependentes do estado. O maior interesse deste é analisar o uso destes modelos para uma aplicação em finanças, ou seja, utilizá-los na construção de regras de mercado.

No Capítulo 2 apresentamos alguns conceitos básicos utilizados no decorrer do todo o texto.

O Capítulo 3 aborda os modelos GARCH e suas extenções. Apresentamos testes para efeitos ARCH, métodos de estimação dos parâmetros dos modelos e algumas ferramentas de diagnóstico dos modelos ajustados. Os modelos GARCH usual e com mudança de regime são descritos no Capítulo 4, com ênfase no estudo do modelo utilizado. Encontram-se neste capítulo as ferramentas de diagnóstico dos ajustes dos modelos. Aplicações a dados reais podem ser encontradas no Capítulo 5. As séries utilizadas para as aplicações são séries de cotações de preços de ações de duas grandes bolsas mundiais (Ibovespa e S\&P500) desde 2003 até 2012.

Finalmente, apresentamos algumas conclusões a respeito do trabalho realizado. 


\section{Capítulo 2}

\section{Conceitos}

Neste capítulo serão apresentados alguns conceitos e definições que servirão de base para o desenvolvimento dos outros capítulos. Mais detalhes podem ser encontrados em Toloi (2006), Fuller (1996) e Hamilton e Susmel (1994).

\subsection{Séries Temporais Estacionárias e Ergódicas}

Um processo estocástico $y=\{y(t, w), t \in T, w \in \Omega\}$ é uma família de variáveis aleatórias (v.a.) definidas num espaço de probabilidade $(\Omega, \Lambda, P)$ e indexadas pelos elementos de um conjunto de parâmetros $T, T \subset[0, \infty)$.

Na realidade, um processo estocástico é uma função de dois argumentos, $y(t, w), t \in T, w \in \Omega$. Para cada $t \in T$, a função $y(t, \cdot)$ é mensurável relativamente a $\Lambda$. Por outro lado, para cada $w \in \Omega$ fixado, obtemos uma função $y(\cdot, w): T \rightarrow \mathbb{R}$ que é chamada trajetória, realização, função amostral do processo ou série temporal.

Para facilitar a notação, representaremos $y(t, w)$ por $y_{t}$.

O processo estocástico $y$ é estacionário de segunda ordem, fracamente estacionário, ou ainda, estacionário em sentido amplo se

- $E\left(y_{t}\right)=\mu$, constante, para todo $t \in T$;

- $\gamma\left(y_{t}, y_{t-j}\right)=E\left[\left(y_{t}-\mu\right)\left(y_{t-j}-\mu\right)\right]=\gamma_{j}$, só depende da defasagem $j$, para todo $t$ e qualquer $j$

- $E\left(y_{t}^{2}\right)<\infty$, para qualquer $t$.

Seja $y_{t}$ um processo estacionário e $\gamma_{j}$ a autocovariância de $y_{t}$ de $j$-ésima ordem ou de lag $j$, calculada para todo $j$. A função de autocovariância $\gamma_{j}$ satisfaz as seguintes propriedades:

- $\gamma_{0}=\operatorname{var}\left(y_{t}\right)>0$;

- $\gamma_{0} \geq\left|\gamma_{j}\right|$

- $\gamma_{-j}=\gamma_{j}$;

- $\gamma_{j}$ é não negativa definida, no sentido que:

$$
\sum_{l=1}^{n} \sum_{k=1}^{n} a_{l} a_{k} \gamma_{j_{l}-j_{k}} \geq 0
$$

para quaisquer números reais $a_{1}, \ldots, a_{n}$ e $j_{1}, \ldots, j_{n}$.

A função de autocorrelação (FAC) de $y_{t}$ é definida por

$$
\rho_{j}=\frac{\operatorname{cov}\left(y_{t}, y_{t-j}\right)}{\sqrt{\operatorname{var}\left(y_{t}\right) \operatorname{var}\left(y_{t-j}\right)}}=\frac{\gamma_{j}}{\gamma_{0}}, j=0, \pm 1, \cdots .
$$


Intuitivamente, um processo estacionário é definido por sua média, variância e função de autocorrelação.

Qualquer função de um processo estacionário também é um processo estacionário, isto é, se $y_{t}$ é estacionária, então $z_{t}=g\left(y_{t}\right)$ também é estacionária, para qualquer função $g(\cdot)$.

A autocovariância amostral de lag $j$ e a autocorrelação amostral de lag $j$ são definidas para processos estacionários por

$$
\begin{aligned}
\widehat{\gamma}_{j} & =\frac{1}{T} \sum_{t=j+1}^{T}\left(y_{t}-\bar{y}\right)\left(y_{t-j}-\bar{y}\right) \\
\widehat{\rho}_{j} & =\frac{\widehat{\gamma}_{j}}{\widehat{\gamma}_{0}}
\end{aligned}
$$

onde $\bar{y}=\frac{1}{T} \sum_{t=1}^{T} y_{t}$ é a média amostral.

Dizemos que um processo estocástico $y_{t}$ é ergódico se os momentos amostrais convergirem em probabilidade para os momentos populacionais, ou seja, $\bar{y} \stackrel{p}{\rightarrow} \mu, \widehat{\gamma}_{j} \stackrel{p}{\rightarrow} \gamma_{j}, \widehat{\rho}_{j} \stackrel{p}{\rightarrow} \rho_{j}$.

\subsubsection{Processos Lineares}

Em situações em que se pretende utilizar modelos para descrever séries temporais, é necessário introduzir suposições simplificadoras, que nos conduza a analisar determinadas classes de processos estocásticos. Assim, podemos ter:

- processos estacionários ou não-estacionários, de acordo com a independência ou não relativamente à origem dos tempos;

- processos normais (Gaussianos) ou não-normais, de acordo com as funções de densidade de probabilidade que caracterizam os processos;

- processos Markovianos ou não-Markovianos, de acordo com a independência dos valores do processo, em dado instante, de seus valores em instantes precedentes.

Mais detalhes encontram-se em Toloi (2006).

Seja $y_{t}$ um processo estocástico. O processo $y_{t}$ pode ser representado por um processo linear, ou processo de médias móveis de ordem infinita, da seguinte forma

$$
\begin{array}{r}
y_{t}=\mu+\sum_{k=0}^{\infty} \psi_{k} \epsilon_{t-k}, \\
\psi_{0}=1, \sum_{k=0}^{\infty} \psi_{k}^{2}<\infty, \\
\epsilon_{t} \sim R B\left(0, \sigma^{2}\right),
\end{array}
$$

onde $\epsilon_{t}$ é um ruído branco. Dizemos que $\left\{\epsilon_{t}, t \in T\right\}$ é um ruído branco se as variáveis aleatórias $\epsilon_{t}$ são não correlacionadas, isto é, $\operatorname{Cov}\left\{\epsilon_{t}, \epsilon_{s}\right\}=0, t \neq s$. Para detalhes ver ?.

Para o processo linear (2.3), pode-se mostrar que

$$
\begin{aligned}
E\left[y_{t}\right] & =\mu, \\
\gamma_{0} & =\operatorname{var}\left(y_{t}\right)=\sigma^{2} \sum_{k=0}^{\infty} \psi_{k}^{2}, \\
\gamma_{j} & =\operatorname{cov}\left(y_{t}, y_{t-1}\right)=\sigma^{2} \sum_{k=0}^{\infty} \psi_{k} \psi_{k+j}, \\
e & \\
\rho_{j} & =\frac{\sum_{k=0}^{\infty} \psi_{k} \psi_{k+j}}{\sum_{k=0}^{\infty} \psi_{k}^{2}} .
\end{aligned}
$$


Portanto, as autocorrelações de qualquer processo estacionário e ergódico $y_{t}$ são determinadas pelos pesos $\left\{\psi_{j}\right\}$ da representação $(2.3)$.

\subsection{Séries Temporais Não Estacionárias}

Uma vez que um processo estacionário possui momentos invariantes, um processo não estacionário deve ter alguma dependência nos momentos. As formas mais comuns de não estacionariedade são causadas pela dependência temporal na média e na variância. Iremos apresentar somente os processos integrados de ordem 1, mais usados em finanças.

Processo Integrados

Chamamos $y_{t}$ de um processo integrado de ordem $1\left(y_{t} \sim I(1)\right)$ se possui a forma

$$
y_{t}=y_{t-1}+u_{t}
$$

onde $u_{t}$ é um processo estacionário. Desta forma, tem-se que a primeira diferença de $y_{t}$ é $y_{t}=u_{t} \mathrm{e}$ é estacionário. Devido a esta propriedade, o processo I (1) é chamado também de processo de diferença estacionária. Uma vez que o processo estacionário $u_{t}$ não precisa sofrer diferenças, é chamado de processo integrado de ordem zero $\left(u_{t} \sim I(0)\right)$.

O processo $y_{t}$ é $I(d)$ se $\Delta^{d} y_{t} \sim I(0)$, ou seja, após $d$ diferenças torna-se $I(0)$. Em séries temporais financeiras raramente são modelados processos $I(d)$ com $d>2$.

\subsubsection{Modelos da família ARIMA}

Modelos $A R(p)$

O modelo autoregressivo de ordem $p(A R(p))$ é dado por

$$
y_{t}-\mu=\phi_{1}\left(y_{t-1}-\mu\right)+\cdots+\phi_{p}\left(y_{t-p}-\mu\right)+\epsilon_{t},
$$

ou, utilizando-se a notação de operadores,

$$
\phi(Z)\left(y_{t}-\mu\right)=\epsilon_{t}
$$

onde $Z$ é o operador de translação para o passado, definido como $Z^{i} y_{t}=y_{t-i}$, e $\Phi(Z)=1-\phi_{1} Z-$ $\cdots-\phi_{p} Z^{p}$ e $\mu=E\left[y_{t}\right]$.

Pode-se mostrar que um processo $A R(p)$ é sempre invertível, e é estacionário e ergódico sob a condição de que as raízes da equação característica,

$$
\Phi(z)=1-\phi_{1} z-\phi_{2} z^{2}-\cdots-\phi_{p} z^{p}=0,
$$

estejam fora do círculo unitário. Uma condição necessária para estacionariedade muito útil na prática é que $\left|\phi_{1}+\cdots+\phi_{p}\right|<1$.

A função de autocovariância de ordem $j$ de um processo $A R(p)$ estacionário é dado por

$$
\gamma_{j}=\phi_{1} \gamma_{j-1}+\phi_{2} \gamma_{j-2}+\cdots+\phi_{p} \gamma_{j-p},
$$

com $\gamma_{0}=\operatorname{var}\left(y_{t}\right)$. A função de autocorrelação de ordem $j$ do processo é dada por

$$
\rho_{j}=\phi_{1} \rho_{j-1}+\phi_{2} \rho_{j-2}+\cdots+\phi_{p} \rho_{j-p} .
$$

Função de Autocorrelação Parcial 
A função de autocorrelação parcial (FACP) é um importante instrumento para ajudar na identificação da ordem $p$ de modelos $A R(p)$. A obtenção da FACP baseia-se na estimação da sequência de modelos $A R(1), A R(2), \ldots, A R(p)$ :

$$
\begin{aligned}
z_{t} & =\phi_{11} z_{t-1}+\epsilon_{1 t} \\
z_{t} & =\phi_{21} z_{t-1}+\phi_{22} z_{t-2}+\epsilon_{2 t} \\
\vdots & \\
z_{t} & =\phi_{p 1} z_{t-1}+\phi_{p 2} z_{t-2}+\cdots+\phi_{p p} z_{t-p}+\epsilon_{p t},
\end{aligned}
$$

em que $z_{t}=y_{t}-\mu$. Os últimos coeficientes em cada $A R(p)\left(\phi_{j j}, j=1, \ldots, p\right)$ são chamados coeficientes de autocorrelação parcial.

Para um processo $A R(p)$, o $p$-ésimo coeficiente de autocorrelação parcial é diferente de zero, e o restante é igual a zero para $j>p$.

Modelos $M A(q)$

O modelo de médias móveis de ordem $q(M A(q))$ tem a forma

$$
y_{t}-\mu=\epsilon_{t}+\theta_{1} \epsilon_{t-1}+\cdots+\theta_{q} \epsilon_{t-q}
$$

onde $\epsilon_{t} \sim R B\left(0, \sigma^{2}\right)$.

Em notação de operadores temos

$$
\left(y_{t}-\mu\right)=\theta(L) \epsilon_{t}
$$

onde $\theta(L)=1+\theta_{1} L+\cdots+\theta_{q} L^{q}$. O modelo $M A(q)$ é estacionário e ergódico desde que $\theta_{1}, \cdots, \theta_{q}$ sejam finitos, e é invertível se todas as raízes da equação característica,

$$
\theta(z)=1+\theta_{1} z+\cdots+\theta_{q} z^{q}=0
$$

estiverem fora do círculo unitário. A propriedade de invertibilidade torna possível escrever o modelo como um $A R(\infty)$.

A função de autocovariância de um processo $M A(q)$ é dada por:

$$
\begin{aligned}
& \gamma_{0}=\sigma^{2}\left(1+\theta_{1}^{2}+\cdots+\theta_{q}^{2}\right) \\
& \gamma_{j}=\left\{\begin{array}{r}
\sigma^{2}\left(\theta_{j}+\theta_{j+1} \theta_{1}+\theta_{j+2} \theta_{2}+\cdots+\theta_{q-j} \theta_{q}\right), \quad \text { para } j=1,2, \ldots, q \\
0, \quad \text { c.c }
\end{array}\right.
\end{aligned}
$$

Observa-se que a FAC de um processo $M A(q)$ é diferente de zero para o lag $q$ e igual a zero para $j>q$. Dessa forma, a FAC pode ser usada como um importante instrumento na identificação deste tipo de processo.

A função de autocorrelação do processo pode ser escrita como

$$
\rho_{j}=\frac{\left(\theta_{j}+\theta_{j+1} \theta_{1}+\theta_{j+2} \theta_{2}+\cdots+\theta_{q-j} \theta_{q}\right)}{1+\theta_{1}^{2}+\cdots+\theta_{q}^{2}} .
$$

A função de autocorrelação parcial de um processo $M A(q)$ invertível apresenta decaimento exponencial. 
Modelos $A R M A(p, q)$

O modelo autoregressivo e de médias móveis $(A R M A(p, q))$ tem a forma

$$
y_{t}-\mu=\phi_{1}\left(y_{t}-\mu\right)+\cdots+\phi_{p}\left(y_{t}-\mu\right)+\epsilon_{t}+\theta_{1} \epsilon_{t-1}+\cdots+\theta_{q} \epsilon_{t-q},
$$

em que $\epsilon_{t} \sim R B\left(0, \sigma^{2}\right)$. Pode ser escrito na forma da regressão com intercepto $c$

$$
y_{t}=c+\phi_{1} y_{t-1}+\cdots+\phi_{p} y_{t-p}+\epsilon_{t}+\theta_{1} \epsilon_{t-1}+\cdots+\theta_{q} \epsilon_{t-q},
$$

ou representado utilizando-se o operador $L$

$$
\phi(L)\left(y_{t}-\mu\right)=\theta(L) \epsilon_{t},
$$

e é estacionário e ergódico se as raízes da equação característica $\phi(z)=0$ estiverem fora do círculo unitário, e é invertível se as raízes da equação característica $\theta(z)=0$ estiverem fora do círculo unitário. Assume-se a condição de que os polinômios $\phi(z)$ e $\theta(z)$ não tenham fatores em comum, pois nesse caso teríamos redundância de parâmetros e a ordem do modelo poderia ser reduzida.

A média de um processo estacionário e ergódico $(A R M A(p, q))$ é igual a

$$
\mu=\frac{c}{1-\phi_{1}-\cdots-\phi_{p}},
$$

e suas autocovariâncias e autocorrelações satisfazem as seguintes relações recursivas

$$
\begin{aligned}
& \gamma_{j}=\phi_{1} \gamma_{j-1}+\phi_{2} \gamma_{j-2}+\cdots+\phi_{p} \gamma_{j-p}, j>q, \\
& \rho_{j}=\phi_{1} \rho_{j-1}+\phi_{2} \rho_{j-2}+\cdots+\phi_{p} \rho_{j-p}, j>q .
\end{aligned}
$$

A forma geral da FAC de um processo $(A R M A(p, q))$ é complicada. Para detalhes, ver Hamilton (1994). Em geral, para o processo $(A R M A(p, q))$, a FAC tem o mesmo comportamento da FAC de um processo $A R(p)$ para $p>q$, e a FACP tem comportamento parecido com a FACP de um processo $M A(q)$ para $q>p$. Dessa forma, ambas FAC e FACP podem apresentar decaimento exponencial.

Na prática, modelos $(A R M A(p, q))$ de ordens altas são difíceis de serem estimados e, por este motivo, são raramente usados para analisar dados financeiros. Segundo Zivot (2003), modelos $(A R M A(p, q))$ de ordens baixas, com $p$ e $q$ menores do que três, são geralmente suficientes na análise de séries temporais financeiras.

\section{Modelos $(A R I M A(p, d, q))$}

A especificação do modelo $(A R M A(p, q))(2.6)$ assume que $y_{t}$ é estacionário e ergódico. Se $y_{t}$ possuir alguma tendência como o preço do barril de petróleo, então $y_{t}$ deve sofrer alguma transformação com o objetivo de eliminar esta tendência.

O modelo $(A R I M A(p, d, q))$ autoregressivo integrado e de médias móveis foi proposto para a modelagem de processos nos quais pelo menos uma raiz da equação característica $\phi(z)=0$ se encontra sobre o círculo unitário. Neste caso o processo é não estacionário na média.

Se houver uma raíz unitária em $y_{t}$, então a primeira diferença $\Delta y_{t}=y_{t}-y_{t-1}$ elimina tal tendência, onde $\Delta=1-L$ é o operador diferença. Se houver duas raízes unitárias em $y_{t}$ então haverá tendência linear em $\Delta y_{t}$, mas a segunda diferença $\Delta^{2} y_{t}=\left(1-2 L+L^{2}\right) y_{t}=y_{t}-2 y_{t-1}+y_{t-2}$ não apresentará tendência. O modelo $(A R I M A(p, d, q))$ apresenta $d$ diferenças para eliminar as tendências e depois é ajustado um modelo $A R M A(p, q)$. 
O modelo $(A R I M A(p, d, q))$ pode ser representado por

$$
\phi(L) \Delta^{d}\left(y_{t}-\mu\right)=\theta(L) \epsilon_{t},
$$

onde $\Delta^{d}=(1-L)^{d}$.

\subsection{Martingal e Sequência de Diferenças de Martingal}

Seja $\left\{y_{t}\right\}$ uma sequência de variáveis aleatórias e seja $I_{t}=\left\{y_{t}, y_{t-1}, \cdots\right\}$ um conjunto de informações baseadas no passado histórico de $\left\{y_{t}\right\}$. A sequência $\left\{y_{t}, I_{t}\right\}$ é chamada de um martingal se

- $I_{t-1} \subset I_{t}$;

- $E\left[\left|y_{t}\right|\right]<\infty$

- $E\left[y_{t} \mid I_{t-1}\right]=y_{t-1}$ (propriedade martingal)

Um exemplo comum de um martingal é o modelo de passeio aleatório

$$
y_{t}=y_{t-1}+\epsilon_{t}, \epsilon_{t} \sim R B\left(0, \sigma^{2}\right),
$$

onde $y_{0}$ é um valor inicial fixado. Se $I_{t}=\left\{y_{t}, \ldots, y_{0}\right\}$ então $E\left[y_{t} \mid I_{t-1}\right]=y_{t-1}$, dado que $E\left[\epsilon_{t} \mid I_{t-1}\right]=$ 0 .

Seja $\left\{\epsilon_{t}\right\}$ uma sequência de variáveis aleatórias com um conjunto de informações associadas a $I_{t}$. A sequência $\left\{\epsilon_{t}, I_{t}\right\}$ é chamada de uma sequência de diferenças de martigal (martingale difference sequence (MDS)) se

- $I_{t-1} \subset I_{t}$;

- $E\left[\epsilon_{t} \mid I_{t-1}\right]=0 ;($ propriedade MDS)

Se $\left\{y_{t}, I_{t}\right\}$ é um martingal, um MDS $\left\{\epsilon_{t}, I_{t}\right\}$ pode ser construído definindo-se

$$
\epsilon_{t}=y_{t}-E\left[y_{t} \mid I_{t-1}\right] .
$$

Por construção, um MDS é um processo não correlacionado.

Para $k>0$ temos

$$
\begin{aligned}
E\left[\epsilon_{t} \epsilon_{t-k}\right] & =E\left[E\left[\epsilon_{t} \epsilon_{t-k} \mid I_{t-1}\right]\right] \\
& =E\left[\epsilon_{t-k} E\left[\epsilon_{t} \mid I_{t-1}\right]\right] \\
& =0 .
\end{aligned}
$$

Embora um processo MDS seja não correlacionado, ele não é necessariamente independente, isto é, pode exitir dependência em momentos de maior ordem de $\epsilon_{t}$.

Processos MDS possuem muitos resultados úteis de convergência (lei de los grandes números, teorema central do limite, etc.) White (1984), Hamilton e Susmel (1994) e Hayashi (2000) apresentam muitos destes resultados para a análise de séries temporais financeiras.

\subsection{Variância de Longo Prazo}

Seja $y_{t}$ uma série temporal estacionária e ergódica. Pelo Teorema Central do Limite

$$
\sqrt{T}(\bar{y}-\mu) \stackrel{d}{\rightarrow} N\left(0, \sum_{j=-\infty}^{\infty} \gamma_{j}\right), \quad T \rightarrow \infty
$$


ou $\bar{y}$ se distribui assintoticamente com distribuição $N\left(\mu, \frac{1}{T} \sum_{j=-\infty}^{\infty} \gamma_{j}\right)$, onde $T$ é o tamanho da amostra, $\bar{y}$ é a média amostral de $y_{t}$ e $\gamma_{j}$ são as autocovariâncias.

A variância de longo prazo (vlp) de $y_{t}$ é dada pela multiplicação de $T$ pela variância assintótica (avar) da média amostral:

$$
\operatorname{vlp}\left(y_{t}\right)=T \cdot \operatorname{avar}(\bar{y})=\sum_{j=-\infty}^{\infty} \gamma_{j} .
$$

Dado que $\gamma_{-j}=\gamma_{j}$, a variância de longo prazo de $y_{t}$ pode ser escrita como

$$
v l p\left(y_{t}\right)=\gamma_{0}+2 \sum_{j=1}^{\infty} \gamma_{j} .
$$

Estimando a Variância de Longo Prazo

Se $y_{t}$ é um processo linear temos que

$$
\sum_{j=-\infty}^{\infty} \gamma_{j}=\sigma^{2}\left(\sum_{j=0}^{\infty} \psi_{j}\right)^{2}=\sigma^{2} \psi(1)^{2},
$$

e, portanto

$$
v \operatorname{lp}\left(y_{t}\right)=\sigma^{2} \psi(1)^{2}
$$

Se $y_{t} \sim A R M A(p, q)$ então

$$
\phi(1)=\frac{1+\theta_{1}+\cdots+\theta_{q}}{1-\phi_{1}-\cdots-\phi_{p}}=\frac{\theta(1)}{\phi(1)}
$$

e, portanto

$$
v l p\left(y_{t}\right)=\frac{\sigma^{2} \theta(1)^{2}}{\phi(1)^{2}} .
$$

Uma estimativa consistente para $v l p\left(y_{t}\right)$ pode ser calculada pela substituição de parâmetros estimados do modelo $(A R M A(p, q))$ apropriado em (2.10). De forma alternativa, pode-se aproximar o modelo $(A R M A(p, q))$ por um processo $\left(A R\left(p^{*}\right)\right)$

$$
y_{t}=c+\phi_{1} y_{t-1}+\cdots+\phi_{p^{*}} y_{t-p^{*}}+\epsilon_{t},
$$

onde $p^{*}$ é escolhido de tal forma que $\epsilon_{t}$ seja não correlacionado. Assim, a estimativa da variância de longo prazo autoregressiva fica dada por

$$
v l p_{A R}\left(y_{t}\right)=\frac{\sigma^{2}}{\phi^{*}(1)^{2}} .
$$

\subsection{Retornos}

Definiremos retornos e log-retornos e apresentaremos suas principais caraterísticas como apresentado em Toloi (2006). Para avaliar o risco de uma carteira de ativos no mercado financeiro é comum medir as variações de preços destes ativos. Na prática, é preferível trabalhar com retornos do que com preços pois os retornos são livres de escala e têm propriedades estatísticas interessantes 
(como estacionariedade e ergodicidade). Os modelos apresentados neste trabalho têm como objetivo modelar a volatilidade dos retornos de séries financeiras, como retornos de preços de ações, por exemplo.

Seja $P_{t}$ o preço de um ativo no instante $t$. A variação de preços entre os instantes $t-1$ e $t$ é dada por $\Delta P_{t}=P_{t}-P_{t-1}$ e a variação relativa de preços ou retorno simples deste ativo é definido por

$$
R_{t}=\frac{P_{t}-P_{t-1}}{P_{t-1}}=\frac{\Delta P_{t}}{P_{t-1}}
$$

De (2.14) temos que $R_{t}=\frac{P_{t}}{P_{t-1}}-1$. Chamamos $1+R_{t}=\frac{P_{t}}{P_{t-1}}$ de retorno bruto simples ou taxa de retorno.

Definimos o retorno composto continuamente, ou log - retorno ou simplesmente retorno, como

$$
r_{t}=\ln \frac{P_{t}}{P_{t-1}}=\ln \left(1+R_{t}\right)=\ln \left(P_{t}\right)-\ln \left(P_{t-1}\right)=p_{t}-p_{t-1},
$$

onde $p_{t}=\ln \left(P_{t}\right)$. Nesse trabalho utilizaremos somente o retorno $r_{t}$ do índice Ibovespa.

\section{Fatos Estilizados sobre os Retornos}

Séries financeiras apresentam algumas características que são comuns a outras séries temporais, como tendências, sazonalidade, pontos influentes (atípicos), heterocedasticidade condicional e nãolinearidade. Os retornos financeiros, por outro lado, apresentam outras características peculiares que outras séries não apresentam. Retornos raramente apresentam tendências ou sazonalidades, com exceção eventualmente de retornos intra-diários, e séries de taxas de câmbio ou taxa de juros que podem apresentar tendências que variam com o tempo. Podemos resumir os principais fatos estilizados relativos a retornos financeiros:

1. retornos são geralmente não autocorrelacionados;

2. os quadrados dos retornos são autocorrelacionados;

3. séries de retornos apresentam agrupamentos de volatilidades ao longo do tempo;

4. a distribuição (não-condicional) dos retornos apresenta caudas mais pesadas do que uma distribuição normal;

5. algumas séries de retornos são não-lineares (respondem de maneira diferente a choques grandes ou pequenos, ou a choques negativos ou positivos).

Utilizaremos essas características para analisar as séries de retornos da Ibovespa. Os conceitos serão importantes ao introduzirmos os modelos de volatilidade no capítulo seguinte. 


\section{Capítulo 3}

\section{Modelos GARCH}

\subsection{Introdução}

O comportamento variável do mercado financeiro é usualmente referido como "volatilidade". A volatilidade tem-se transformado num conceito muito importante em diferentes áreas da teoria e da prática financeira, como gerenciamento de risco, seleção de portifólio, derivação de preços, etc. Em termos estatísticos, a volatilidade é medida pela variância ou pelo desvio padrão condicional.

Nesse capítulo vamos introduzir a classe dos modelos GARCH (Generalized Autoregressive Conditional Heteroskedasticity) desenvolvidos por Engle (1989), Bollerslev (1986), Nelson (1991), e outros, que são capazes de modelar a volatilidade variando no tempo e de capturar muitos dos fatos estilizados do comportamento da volatilidade observada em séries temporais financeiras. Também será apresentado o ajuste do modelo GARCH para os retornos do Ibovespa e os métodos de avaliação desse ajuste.

\subsection{Modelos ARCH}

O Índice Bovespa é composto pelas ações mais negociadas na bolsa de São Paulo e os pesos das ações mudam ao longo do tempo, mas as que tem maior peso são Petrobras, Eletrobras, etc. Na Figura 3.1 temos os índices e os log-retornos diários do índice Bovespa, no período de 2 de janeiro de 2003 a 9 de abril de 2012, para um total de 2294 observações. Pode-se perceber que os períodos de alta (crise em 2008) e de baixa volatilidade estão agrupados (fato estilizado). Essa tendência é típica de muitas séries temporais financeiras. Para confirmar este comportamento são apresentadas na Figura 3.2 as funções de autocorrelação dos retornos e dos retornos ao quadrado da série de log retornos do índice Bovespa.

Observando os gráficos das funções de autocorrelação percebemos que não há autocorrelações significantes para qualquer defasagem da série dos log-retornos. Por outro lado, a autocorrelação da série dos log-retornos ao quadrado se mostra significante pelo menos até a defasagem 30 . Uma vez que os log-retornos ao quadrado medem o momento de segunda ordem da série original, este resultado indica que a variância dos log-retornos da série índice Bovespa condicionada ao passado histórico pode sofrer alterações ao longo do tempo, ou seja, a série de log-retornos pode mostrar a presença de heteroscedasticidade condicional.

A heteroscedasticidade condicional da série dos log-retornos ao quadrado pode ser modelada usando um processo autorregressivo simples (AR) para resíduos ao quadrado. Seja $y_{t}$ uma série temporal estacionária tal como log-retornos financeiros e não exista autocorrelação significante em $y_{t}$ então $y_{t}$ pode ser expresso como sua média acrescida de um ruído branco:

$$
y_{t}=c+\epsilon_{t}
$$

onde $c$ é a média de $y_{t}$ e $\epsilon_{t}$ são erros não correlacionados com média zero. 

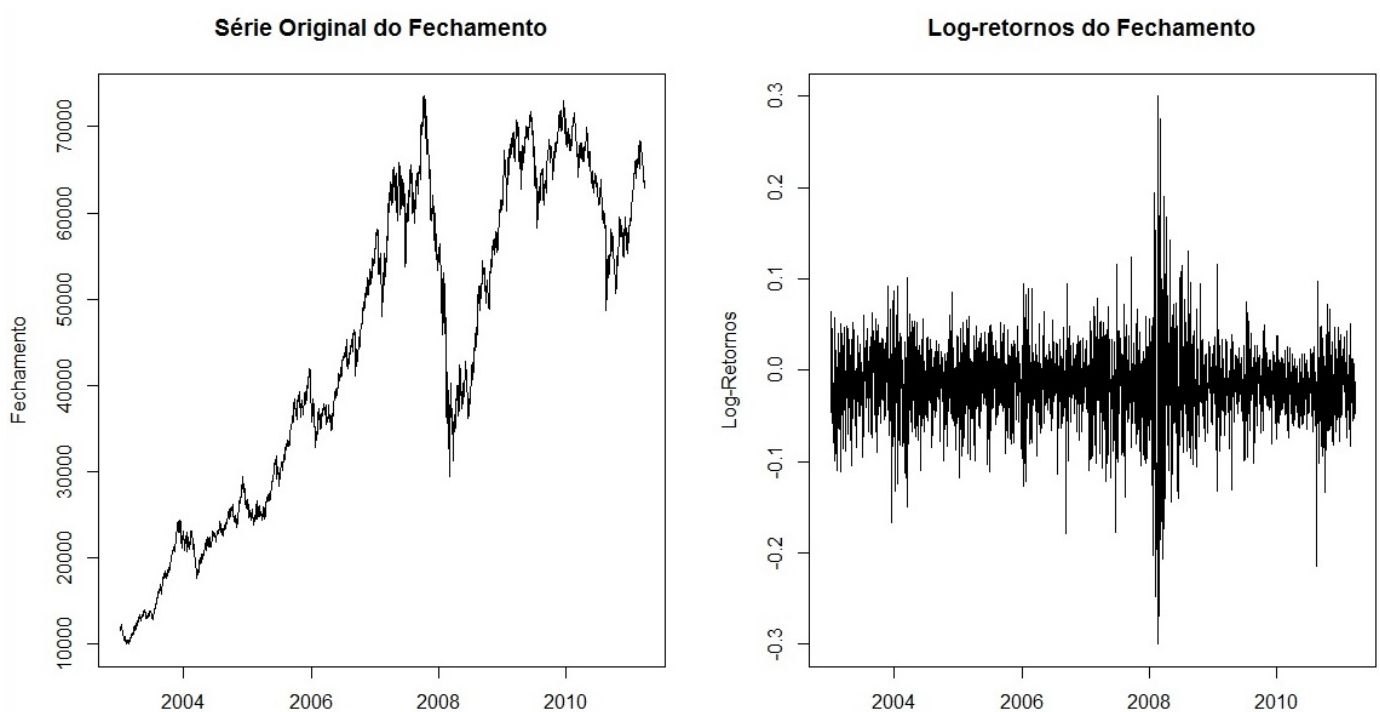

Figura 3.1: Gráfico da Série do indice Bovespa e os Log-retornos.

Series Inret

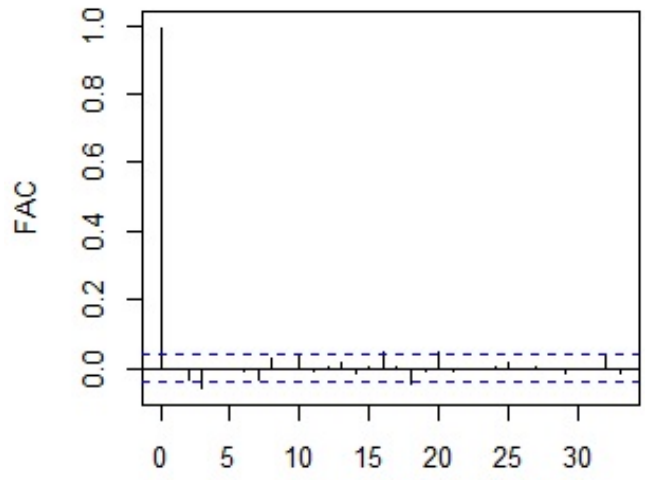

lag

\section{Series Inret2}

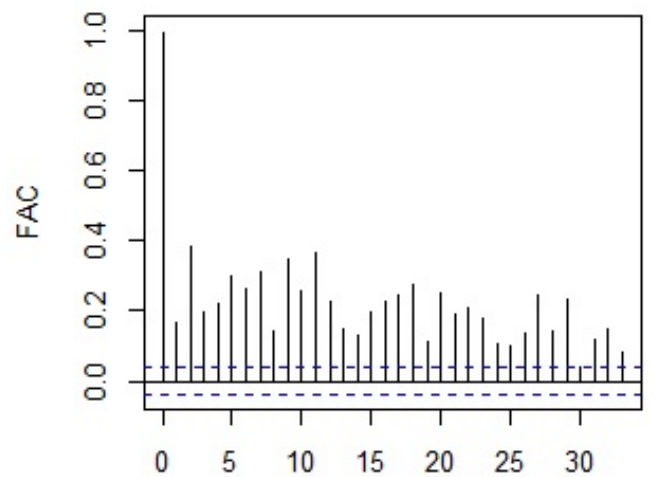

lag
Series Inret

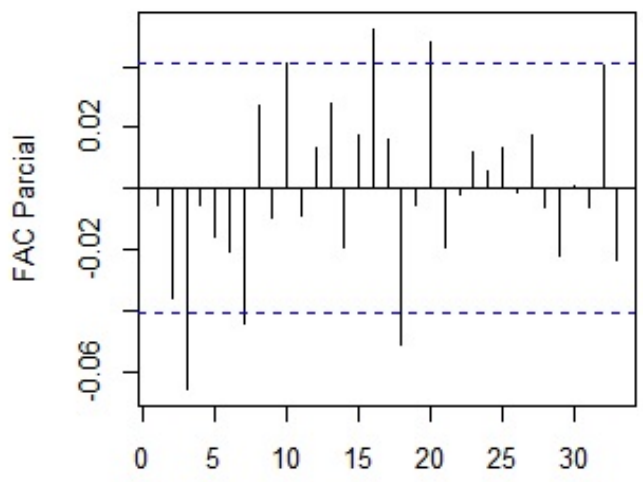

lag

Series Inret2

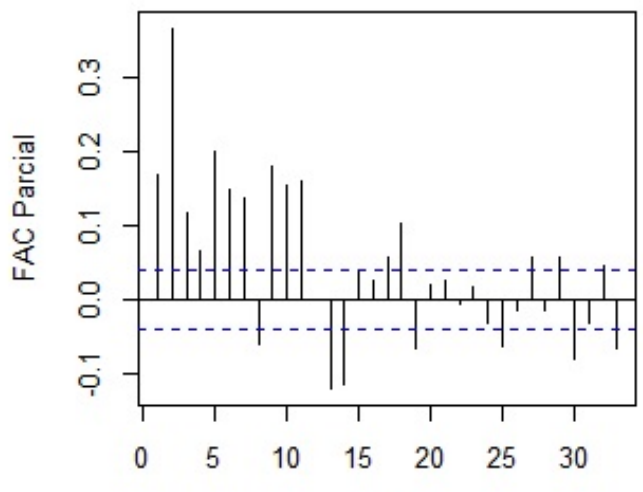

lag

Figura 3.2: Gráfico da função de autocorrelação e autocorrelação parcial da Série dos log-retornos do índice Bovespa 
Para incluir o efeito da hereroscedasticidade condicional, seja

$$
\operatorname{Var}_{t-1}\left(\epsilon_{t}\right)=\sigma_{t}^{2}
$$

em que $\operatorname{Var}_{t-1}(\cdot)$ é a variância condicional à informação até o tempo $t-1$, e

$$
\sigma_{t}^{2}=a_{0}+a_{1} \epsilon_{t-1}^{2}+\cdots+a_{p} \epsilon_{t-p}^{2}
$$

em que $p$ indica o lag mais distante do instante $t$, de modo que as informações em $p$ ainda influenciam $\sigma_{t}^{2}$. Como $\epsilon_{t}$ tem média zero, $\operatorname{Var}_{t-1}\left(\epsilon_{t}\right)=E_{t-1}\left(\epsilon_{t}^{2}\right)=\sigma_{t}^{2}$, a equação acima pode ser reescrita como:

$$
\epsilon_{t}^{2}=a_{0}+a_{1} \epsilon_{t-1}^{2}+\cdots+a_{p} \epsilon_{t-p}^{2}+u_{t},
$$

em que $u_{t}=\epsilon_{t}^{2}-E_{t-1}\left(\epsilon_{t}^{2}\right)$ é um processo diferença de martingal com média zero, mas com variância não constante. A equação (3.3) representa um processo $A R(p)$ para $\epsilon_{t}^{2}$, e o modelo em (3.1) e (3.2) é conhecido como o modelo autorregressivo com heteroscedasticidade condicional de Engle (1989), que é usualmente chamado de modelo $A R C H(p)$.

Uma formulação equivalente para o modelo $A R C H(p)$ é

$$
\begin{aligned}
y_{t} & =c+\epsilon_{t} \\
\epsilon_{t} & =z_{t} \sigma_{t} \\
\sigma_{t}^{2} & =a_{0}+a_{1} \epsilon_{t-1}^{2}+\cdots+a_{p} \epsilon_{t-p}^{2}
\end{aligned}
$$

em que $z_{t}$ é uma sequência de variáveis aleatórias independentes e identicamente distribuidas com média 0 e variância 1 com uma distribuição especificada. No modelo ARCH básico a distribuição de $z_{t}$ é a normal padrão (iid). Entretanto, também é usual adotar distribuições com caudas mais pesadas como a t-Student e a distribuição do erro generalizada.

\subsection{Testes para efeitos com Heteroscedasticidade Condicional}

Antes de estimar um modelo ARCH para uma série temporal financeira, é interessante testar a presença de efeitos ARCH nos resíduos padronizados. Se não houver efeitos ARCH nos resíduos, então o modelo ARCH é desnecessário e mal especificado.

Uma vez que o modelo ARCH pode ser escrito como um modelo AR em termos de resíduos quadráticos como em (3.3), um teste simples assintótico de Multiplicador de Lagrange (LM) para modelos ARCH pode ser construído baseado na regressão auxiliar (3.3). Sob a hipótese nula de que não há efeitos ARCH: $a_{1}=a_{2}=\cdots=a_{p}=0$, a estatística do teste terá distribuição assintótica qui quadrado:

$$
L M=T \cdot R^{2} \stackrel{a}{\rightarrow} \chi_{(p)}^{2},
$$

onde $T$ é o tamanho da amostra e $R^{2}$ é o quadrado do coeficiente de correlação múltipla da regressão auxiliar da equação (3.3) usando os resíduos estimados, ou seja, substituindo $\sigma_{t}^{2}$ por $\widehat{\epsilon}_{t}^{2}$ e os erros defasados $\epsilon_{t-j}$ por $\widehat{\epsilon}_{t-j}$

Outro teste muito utilizado para verificar a correlação entre os resíduos ao quadrado é o teste de Box-Pierce - Ljung (Box e Pierce, 1970) para $\epsilon_{t}^{2}$. Apesar desse teste não detectar quebras estruturais específicas no comportamento de um ruído branco, pode indicar se esses valores são muito altos. Uma modificação deste teste foi proposta por Ljung e Box (1978).

Esse teste tem $H_{0}: \rho_{1}=\cdots=\rho_{K}=0$ onde $\rho_{k}$ é a autocorrelação de lag $k$ dos resíduos $\widehat{\epsilon}_{t}$. A 
estatística do teste é

$$
Q(K)=n(n+2) \sum_{k=1}^{K} \frac{\widehat{r}_{k}^{2}}{(n-k)},
$$

terá sob $H_{0}$ uma distribuição $\chi^{2}$ com $K-p-q$ graus de liberdade, em que, $p$ e $q$ indicam as ordens do modelo $\operatorname{GARCH}(\mathrm{p}, \mathrm{q})$ e $\mathrm{K}$ refere-se às primeiras $K$ correlações amostrais.

\subsection{Modelo GARCH}

Como foi mostrado anteriormente, uma maneira adequada para modelar a volatilidade é a utilização dos modelos $\mathrm{ARCH}$. No entanto, tem-se que na prática geralmente é preciso um número grande de lags $p$ para se obter um bom ajuste no modelo, o que implica um número grande de parâmetros. Um modelo mais parcimonioso foi proposto por Bollerslev (1986) e substitui o modelo AR em (3.2) pela formulação:

$$
\sigma_{t}^{2}=a_{0}+\sum_{i=1}^{p} a_{i} \epsilon_{t-i}^{2}+\sum_{j=1}^{q} b_{j} \sigma_{t-j}^{2},
$$

onde $a_{0}>0$ e os coeficientes $a_{i}(i=1, \cdots, p)$ e $b_{j}(j=1, \cdots, q)$ são todos não negativos para garantir que a variância condicional $\sigma_{t}^{2}$ seja sempre positiva. O modelo (3.4) junto com (3.1) é conhecido como o modelo $\operatorname{GARCH}(p, q)$ "generalized $\mathrm{ARCH} "$. Quando $q=0$, o modelo GARCH se reduz ao modelo $\operatorname{ARCH}(p)$.

No modelo $\operatorname{GARCH}(p, q)$ a variância condicional de $\epsilon_{t}^{2}, \sigma_{t}^{2}$, depende do quadrado dos erros nos $p$ períodos anteriores e da variância condicional nos $q$ períodos anteriores.

\section{Representação ARMA do Modelo GARCH}

Assim como um modelo ARCH pode ser escrito como um modelo AR em termos de erros quadráticos, um modelo GARCH poder ser escrito na forma de um modelo ARMA de erros quadráticos. Considere o modelo GARCH $(1,1)$ :

$$
\sigma_{t}^{2}=a_{0}+a_{1} \epsilon_{t-1}^{2}+b_{1} \sigma_{t-1}^{2}
$$

Sendo $E_{t-1}\left(\epsilon_{t}^{2}\right)=\sigma_{t}^{2}$, a equação acima pode ser escrita como:

$$
\epsilon_{t}^{2}=a_{0}+\left(a_{1}+b_{1}\right) \epsilon_{t-1}^{2}+u_{t}-b_{1} u_{t-1}
$$

que é um modelo $\operatorname{ARMA}(1,1)$ onde $u_{t}=\epsilon_{t}^{2}-E_{t-1}\left(\epsilon_{t}^{2}\right)$ é ruído branco de média zero e variância não constante.

Dada a representação ARMA de um modelo GARCH, muitas propriedades do modelo GARCH seguem facilmente da correspondência de um processo ARMA para $\epsilon_{t}^{2}$. Por exemplo, para o modelo $\operatorname{GARCH}(1,1)$ ser estacionário é preciso $a_{1}+b_{1}<1$. Assumindo a estacionariedade do modelo $\operatorname{GARCH}(1,1)$, pode-se mostrar que a variância incondicional de $\epsilon_{t}$ é dada por $\operatorname{Var}\left(\epsilon_{t}\right)=E\left(\epsilon_{t}^{2}\right)=$ $\frac{a_{0}}{1-a_{1}-b_{1}}$, pois de (3.6) temos:

$$
E\left(\epsilon_{t}^{2}\right)=a_{0}+\left(a_{1}+b_{1}\right) E\left(\epsilon_{t-1}^{2}\right)
$$

e portanto

$$
E\left(\epsilon_{t}^{2}\right)=a_{0}+\left(a_{1}+b_{1}\right) E\left(\epsilon_{t}^{2}\right),
$$

baseado na suposição de que $\epsilon_{t}^{2}$ é estacionário. 
Para o modelo GARCH(p,q) em (3.4), os erros quadráticos $\epsilon_{t}^{2}$ têm comportamento de um processo $\operatorname{ARMA}(\max (\mathrm{p}, \mathrm{q}), \mathrm{q})$. A estacionariedade requer que $\sum_{i=1}^{p} a_{i}+\sum_{j=1}^{q} b_{j}<1$ e a variância incondicional de $\epsilon_{t}$ é dada por

$$
\bar{\sigma}^{2}=\operatorname{Var}\left(\epsilon_{t}\right)=\frac{a_{0}}{1-\left(\sum_{i=1}^{p} a_{i}+\sum_{j=1}^{q} b_{j}\right)} .
$$

\subsection{Modelo GARCH e Fatos Estilizados}

Na prática, existem alguns "fatos estilizados"sobre a volatilidade de séries temporais financeiras. Bollerslev, Engle e Nelson (1994) apresentam uma visão completa sobre esses fatos. Usando a representação ARMA dos modelos GARCH é possível mostrar que o modelo GARCH é capaz de explicar muitos desses fatos estilizados. Nessa seção serão detalhados dois importantes fatos estilizados: agrupamento de volatilidades (volatility clustering) e caudas pesadas.

No modelo GARCH $(1,1)$ dado em $(3.5)$ a estimativa do coeficiente $b_{1}$ é normalmente um número próximo de 0,9 para séries temporais financeiras diárias ou semanais. Dessa maneira, temos que valores grandes de $\sigma_{t-1}^{2}$ são seguidos por valores grandes de $\sigma_{t}^{2}$, e valores pequenos de $\sigma_{t-1}^{2}$, por sua vez, são seguidos por valores pequenos de $\sigma_{t}^{2}$. Seguindo o mesmo raciocínio para a representação ARMA em (3.6), temos que grandes/pequenas mudanças em $\epsilon_{t-1}^{2}$ são seguidas por grandes/pequenas mudanças em $\epsilon_{t}^{2}$.

É fato conhecido que a distribuição de muitas séries temporais financeiras geralmente possuem caudas mais pesadas que a distribuição normal, ou seja, grandes mudanças ocorrem com mais frequência do que em uma distribuição normal. Bollerslev (1986) descreve as condições para a existência do momento de quarta ordem de um processo $\operatorname{GARCH}(1,1)$. Assumindo que o momento de quarta ordem exista, Bollerslev (1986) mostra que a curtose de um processo GARCH(1,1) é maior do que 3, que é a curtose de uma distribuição normal. He (1999a) e He (1999b) extenderam o resultado de Bollerslev para os modelos GARCH(p,q). Portanto, um modelo GARCH pode reproduzir as caudas pesadas observadas em séries temporais financeiras.

\subsection{Estimação de Modelos GARCH}

O objetivo dessa seção é estimar um modelo GARCH. Conforme apresentado anteriormente, o modelo geral $\operatorname{GARCH}(\mathrm{p}, \mathrm{q})$ é da forma

$$
\begin{aligned}
y_{t} & =c+\epsilon_{t} \\
\epsilon_{t} & =z_{t} \sigma_{t} \\
\sigma_{t}^{2} & =a_{0}+\sum_{i=1}^{p} a_{i} \epsilon_{t-i}^{2}+\sum_{j=1}^{q} b_{j} \sigma_{t-j}^{2},
\end{aligned}
$$

para $t=1, \cdots, T$, onde $\sigma_{t}^{2}=\operatorname{Var}_{t-1}\left(\epsilon_{t}\right)$.

Serão introduzidos dois tipos de estimadores para os parâmetros $c, a_{0}, a_{i}$ e $b_{j}$ : estimador de máxima verossimilhança condicional e estimador de mínimos desvios absolutos. O primeiro é o estimador mais conhecido e é bastante usado na área de operações financeiras. O estimador de mínimos desvios absolutos é interessante quando tratamos de erros com caudas pesadas. Nas analises desse trabalho utilizaremos apenas o estimador de máxima verossimilhança condicional. 


\subsubsection{Estimador de Máxima Verossimilhança Condicional}

Assim como na estimação dos modelos ARMA, os estimadores mais usados para modelos AR$\mathrm{CH} / \mathrm{GARCH}$ são aqueles derivados de uma função Gaussiana de máxima verossimilhança (condicional). Por exemplo, se $z_{t}$ em (3.8) tem distribuição normal e $q=0$ (ou seja, um modelo ARCH puro), a função log-verossimilhança condicional negativa baseada nas observações $\epsilon_{1}, \cdots, \epsilon_{T}$, ignorando-se as constantes, é igual a

$$
l\left(\sigma_{t}^{2} \mid \epsilon_{1}, \cdots, \epsilon_{T}\right)=-\sum_{t=p+1}^{T}\left(\log \sigma_{t}^{2}+\epsilon_{t}^{2} / \sigma_{t}^{2}\right)
$$

onde $\sigma_{t}^{2}=a_{0}+\sum_{i=1}^{p} a_{i} \epsilon_{t-i}^{2}$. Os estimadores de máxima verossimilhança são obtidos minimizandose a função em (3.9). Podemos perceber que esta função de verossimilhança é baseada na função densidade de probabilidade condicional de $\epsilon_{p+1}, \cdots, \epsilon_{T}$, dados, $\epsilon_{1}, \cdots, \epsilon_{p}$, uma vez que a função de densidade incondicional, que envolve a densidade conjunta de $\epsilon_{1}, \cdots, \epsilon_{p}$, é mais difícil de ser obtida.

Para o modelo GARCH geral, ou seja, $q>0$ no modelo (3.4) a variância condicional $\sigma_{t}^{2}$ não pode ser expressa em termos de um número finito de observações passadas $\epsilon_{t-1}, \epsilon_{t-2}, \cdots$. Por indução, podemos escrever

$$
\sigma_{t}^{2}=\frac{a_{0}}{1-\sum_{j=1}^{q} b_{j}}+\sum_{i=1}^{p} a_{i} \epsilon_{t-i}^{2}+\sum_{i=1}^{p} a_{i} \sum_{k=1}^{\infty} \sum_{j_{1}=1}^{q} \cdots \sum_{j_{k}=1}^{q} b_{j_{1}} \cdots b_{j_{k}} \epsilon_{t-i-j_{1}-\cdots-j_{k}}^{2},
$$

onde a soma múltipla desaparece quando $q=0$. Nota-se que a soma múltipla acima converge com probabilidade 1 desde que cada $a_{i}$ e $b_{j}$ seja não negativo, e desde que o valor esperado da série múltipla seja finito. Na prática, a expressão (3.10) é substituída por uma versão truncada

$$
\begin{array}{r}
\widetilde{\sigma}_{t}^{2}=\frac{a_{0}}{1-\sum_{j=1}^{q} b_{j}}+\sum_{i=1}^{p} a_{i} \epsilon_{t-i}^{2}+ \\
+\sum_{i=1}^{p} a_{i} \sum_{k=1}^{\infty} \sum_{j_{1}=1}^{q} \cdots \sum_{j_{k}=1}^{q} b_{j_{1}} \cdots b_{j_{k}} \\
\epsilon_{t-i-j_{1}-\cdots-j_{k}}^{2} I\left(t-i-j_{1}-\cdots-j_{k} \geq 1\right), t>p .
\end{array}
$$

Temos que, quando $q=0, \widetilde{\sigma}_{t}^{2}=\sigma_{t}^{2}=a_{0}+\sum_{i=1}^{p} a_{i} \epsilon_{t-i}^{2}$.

Considere a notação $a=\left(a_{1}, \cdots, a_{p}\right)^{T}$ e $b=\left(b_{1}, \cdots, b_{q}\right)^{T}$. O estimador de máxima verossimilhança (condicional) $\left(\widehat{a}_{0}, \widehat{a}, \widehat{b}\right)$ é definido maximizando-se a expressão

$$
l_{\nu}\left(a_{0}, a, b\right)=-\sum_{t=\nu}^{T}\left(\log {\widetilde{\sigma_{t}}}^{2}+\epsilon_{t}^{2} /{\widetilde{\sigma_{t}}}^{2}\right)
$$

onde $\nu>p$ é um número inteiro. Em geral $\nu=p+1$, onde $\nu$ são os graus de liberdade.

Supondo que $f(\cdot)$ seja a função densidade de probabilidades de $z_{t}$ conhecida, temos que os estimadores de máxima verossimilhança são obtidos maximizando-se

$$
l_{\nu}\left(a_{0}, a, b\right)=-\sum_{t=\nu}^{T}\left\{\log {\widetilde{\sigma_{t}}}^{2}-2 \log f\left(\epsilon_{t} / \widetilde{\sigma_{t}}\right)\right\},
$$

em vez de maximizar a função (3.12). Além da distribuição normal, algumas distribuições frequentemente usadas são:

- distribuição $t$ com $\nu$ graus de liberdade com densidade apresentada em Marcucci (2005): 


$$
f\left(\epsilon_{t}\right)=\frac{\Gamma\left(\frac{\nu+1}{2}\right)}{\sqrt{\pi} \Gamma\left(\frac{\nu}{2}\right)}(\nu-2)^{-\frac{1}{2}}\left(h_{t}\right)^{-\frac{1}{2}}\left[1+\frac{\epsilon_{t}^{2}}{h_{t}(\nu-2)}\right]^{-\frac{(\nu+1)}{2}} ; \epsilon_{t} \in \Re,
$$

onde $\nu>2$ pode ser tratado como um parâmetro contínuo.

- distribuição Gaussiana generalizada com densidade apresentada em Marcucci (2005):

$$
f\left(\epsilon_{t}\right)=\nu\left\{\lambda 2^{-\frac{2}{\nu}} \Gamma(1 / \nu)\right\}^{-1} \exp \left\{-\frac{1}{2}\left|\frac{x}{\lambda}\right|^{\nu}\right\}
$$

onde $\lambda=\left\{2^{-\frac{2}{\nu}} \Gamma\left(\frac{1}{\nu}\right) / \Gamma\left(\frac{3}{\nu}\right)\right\}^{1 / 2}$ e $0<\nu<2$.

Quando $\nu=1$, a distribuição Gaussiana generalizada se reduz à função $f(x)=\exp \{-\sqrt{2}|x|\} / \sqrt{2}$. Todas as distribuições acima foram normalizadas para ter média 0 e variância 1 , e todas elas possuem caudas mais pesadas do que a distribuição normal. Para comparar dois ou mais modelos ajustados, usamos os valores AIC e BIC para cada um dos modelos ajustados, que são calculados da seguinte maneira:

$$
A I C=l_{\nu}\left(\widehat{a}_{0}, \widehat{a}, \widehat{b}\right)+2(p+q+1)
$$

e

$$
B I C=l_{\nu}\left(\widehat{a}_{0}, \widehat{a}, \widehat{b}\right)+2(p+q+1) \log (T-\nu+1),
$$

onde $l_{\nu}(\cdot)$ segue de $(3.13)$.

\subsubsection{Estimadores de Mínimos Desvios Absolutos}

O estimador apresentado anteriormente é derivado da maximização da verossimilhança Gaussiana ou de uma aproximação da verossimilhança Gaussiana. Sabe-se que os $L_{1}$-estimadores são mais robustos do que os $L_{2}$-estimadores, no que diz respeito às distribuições com caudas pesadas. Algumas evidências empíricas sugerem que este método é mais apropriado para séries financeiras pois estas apresentam caudas mais pesadas que a distribuição normal. Para detalhes ver Mandelbrot (1963), Fama (1965), Mittnik (1998) e Mittnik (2000).

A ideia deste estimador implica uma reparametrização do modelo (3.4), onde $E\left(z_{t}\right)=0$ e a mediana de $z_{t}^{2}$, ao invés da variância de $z_{t}$, é igual a 1 . Sob essa nova formulação, os parâmetros $a_{0}$ e $a_{i}$ 's diferem daqueles da formulação original por um fator constante, enquanto os parâmetros $b_{j}$ 's não se alteram. Seja

$$
\epsilon_{t}^{2} / \sigma_{t}^{2}=1+e_{t, 1}
$$

onde $e_{t, 1}=\left(z_{t}^{2}-1\right)$, que possui mediana igual a zero. Assim, temos que o estimador de mínimos desvios absolutos $\widehat{\theta_{1}}$, que é um $L_{1}$-estimador baseado na regressão (3.16), pode ser obtido minimizando-se

$$
\sum_{t=\nu}^{T}\left|\epsilon_{t}^{2} \widetilde{\sigma_{t}^{2}}-1\right|
$$

onde $\widetilde{\sigma}_{t}^{2}$ é definido em (3.12) e $\nu=p+1$ se $q=0$ e $\nu>p+1$ se $q>0$. Peng. L. (2003) mostraram que este estimador é viesado. Para resolver este problema, Peng e Yao (2003) definiram uma forma modificada para o estimador de mínimos desvios absolutos $\left(\widehat{\theta_{2}}\right)$, que resulta da minimização da 
expressão:

$$
\sum_{t=\nu}^{T}\left|\log \left(\epsilon_{t}^{2}\right)-\log \left(\widetilde{\sigma}_{t}^{2}\right)\right|,
$$

motivados pelo modelo de regressão

$$
\log \left(\epsilon_{t}^{2}\right)=\log \left(\sigma_{t}^{2}\right)+e_{t, 2},
$$

onde os erros $e_{t, 2}=\log \left(z_{t}^{2}\right)$ são i.i.d com mediana zero.

Peng e Yao (2003) demonstraram que sob condições muito amenas, os estimadores de mínimos desvios absolutos são assintoticamente normais com razão de convergência padrão $T^{1 / 2}$, independentemente do fato de a distribuição de $z_{t}$ possuir caudas pesadas ou não. Esta é uma diferença marcante entre estes estimadores e os estimadores de máxima verossimilhança condicional derivados de (3.13), que podem apresentar convergência lenta quando $z_{t}$ possui caudas pesadas.

Outro estimador proposto por Peng e Yao (2003) foi motivado pela equação de regressão

$$
\epsilon_{t}^{2}=\sigma_{t}^{2}+e_{t, 3},
$$

onde $e_{t, 3}=\sigma_{t}^{2}\left(z_{t}-1\right)$ possui mediana igual a zero. Assim, o estimador $\widehat{\theta_{3}}$ é obtido minimizando-se:

$$
\sum_{t=\nu}^{T}\left|\epsilon_{t}^{2}-\widetilde{\sigma_{t}^{2}}\right| .
$$

Intuitivamente, é preferível usar o estimador $\widehat{\theta_{2}}$ ao $\widehat{\theta_{3}}$ pois os termos de erros $e_{t, 2}$ do modelo de regressão (3.20) são independentes e identicamente distribuídos enquanto que os erros $e_{t, 3}$ do modelo (3.21) não são independentes.

Em Peng. L. (2003) foram realizadas comparações entre os três estimadores de mínimos desvios absolutos com o estimador de máxima verossimilhança Gaussiana, $\widehat{\theta_{M L}}$, para modelos simulados $\operatorname{ARCH}(2)$ e $\operatorname{GARCH}(1,1)$, tomando os erros $z_{t}$ com distribuição normal padrão ou $t$-Student padronizada com $d=3$ ou $d=4$ graus de liberdade. A performance do estimador de máxima verossimilhança Gaussiana piora conforme as caudas da distribuição dos erros tendem a ser mais pesadas. Entretanto, esse comportamento não ocorre para os estimadores de mínimos desvios absolutos uma vez que estes apresentam-se mais robustos para caudas pesadas.

Para mais detalhes encontram-se em Peng. L. (2003).

\subsection{Diagnóstico de Modelos GARCH}

As seções anteriores apresentaram métodos para estimação dos modelo GARCH. Para diagnosticar os modelos ajustados são usados basicamente dois métodos: a análise gráfica e a análise inferencial.

$\mathrm{Na}$ análise das estatísticas resumo, podemos analisar os erros padrão e os níveis descritivos (valores p) das estatísticas para avaliar se os coeficientes do modelo são estatisticamente iguais a zero.

Além disso, existem testes variados para os resíduos padronizados $\widehat{\epsilon}_{t} / \widehat{\sigma}_{t}$. Para a série de log-retornos dos preços do índice Bovespa, as Tabelas 3.1 e 3.2 apresentam os coeficientes ajustados por meio do método de máxima verossimilhança condicional e alguns testes para resíduos padronizados do modelo GARCH(1,1): testes de normalidade Jarque (1980) e Shapiro (1965), teste de Ljung-Box para resíduos padronizados e para o quadrado dos resíduos padronizados e teste do multiplicador de Lagrange para efeito ARCH nos resíduos padronizados.

No teste Ljung-Box para os resíduos padronizados, não rejeitamos a hipótese nula de autocorrelação $(p=0,465)$. Por outro lado, no teste Ljung-Box para o quadrado dos resíduos padronizados, não rejeitamos a hipótese da não existência de autocorrelação $(p=0,465)$. Portanto, o modelo 
captura com sucesso a estrutura de correlação serial na variância condicional mas não obtivemos um resultado satisfatório para a média condicional.

Além dos testes para autocorrelação nos resíduos padronizados, pode-se aplicar o teste do multiplicador de Lagrange para efeitos ARCH nos resíduos padronizados para verificar se existe algum efeito ARCH. Não rejeitamos a hipótese nula da não existência de efeitos $\mathrm{ARCH}(p=0,3117)$.

Tabela 3.1: Coeficientes Estimados do modelo GARCH(1,1)

\begin{tabular}{lllll}
\hline Coeficiente & Valor Estimado & Erro Padrão & Estat t & Valor p \\
\hline C & 0,10800 & 0,03196 & 3,379 & 0,0007 \\
A & 0,06877 & 0,01814 & 3,792 & 0,0001 \\
ARCH(1) & 0,07383 & 0,01050 & 7,028 & 0,0000 \\
GARCH(1) & 0,90369 & 0,01354 & 66,760 & 0,0002 \\
\hline
\end{tabular}

O modelo GARCH básico assume distribuição normal para os erros $\epsilon_{t}$. Se o modelo está corretamente especificado então os resíduos padronizados $\hat{\epsilon}_{t} / \hat{\sigma}_{t}$ devem se comportar como uma variável aleatória normal padrão. Os testes de normalidade Jarque-Bera e Shapiro-Wilks levam a outras conclusões, pois ambos rejeitam a suposição de normalidade $(p<0,0001)$. Para comparar os resíduos padronizados com os valores esperados se esses tivessem distribuição normal, construímos o gráfico quantil quantil (qqplot) apresentado na Figura 3.3. Nele podemos perceber que nas caudas há um significante desvio no gráfico de dispersão com relação à linha que corresponde ao caso de quantis normais, e portanto a suposição de normalidade dos resíduos não é apropriada.

Tabela 3.2: Testes para diagnóstico do modelo $\operatorname{GACH}(1,1)$ ajustado

\begin{tabular}{lll}
\hline Testes de Normalidade: & & \\
\hline Jarque-Bera & P-valor & \\
110,0797 & $<0,0001$ & \\
Shapiro-Wilk & P-valor & \\
0,9921 & $<0,0001$ & \\
\hline \hline Teste Ljung-Box para os resíduos padronizados: & & \\
\hline Estatística & P-valor & $\chi^{2}$-g.l \\
9,7236 & 0,4651 & 10 \\
\hline \hline Teste Ljung-Box para o quadrado dos resíduos padronizados: & & \\
\hline Estatística & P-valor & $\chi^{2}$-g.l \\
13,0993 & 0,2182 & 10 \\
\hline \hline Teste do multiplicador de Lagrange para os resíduos padronizados: & & \\
\hline Estatística & P-valor & $\chi^{2}$-g.l \\
13,8301 & 0,3117 & 10 \\
\hline
\end{tabular}

Outros gráficos também podem ser utilizados para visualizar o ajuste do modelo. Por exemplo, na Figura 3.4 apresentamos a função de autocorrelação dos retornos padronizados e do quadrado dos retornos padronizados. 


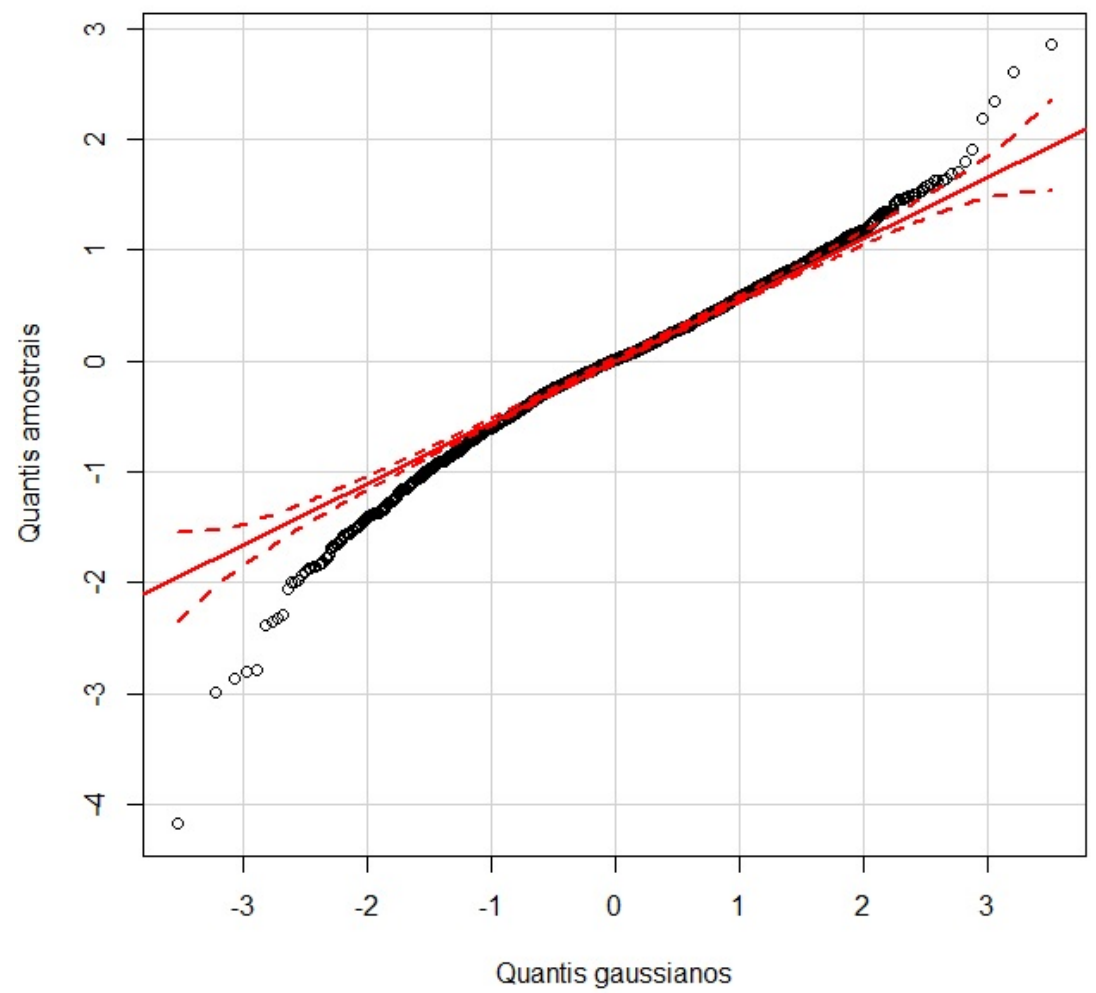

Figura 3.3: Gráfico de quantis dos Resíduos padronizados do modelo $\operatorname{GARCH}(1,1)$ da série de retornos do Ibovespa
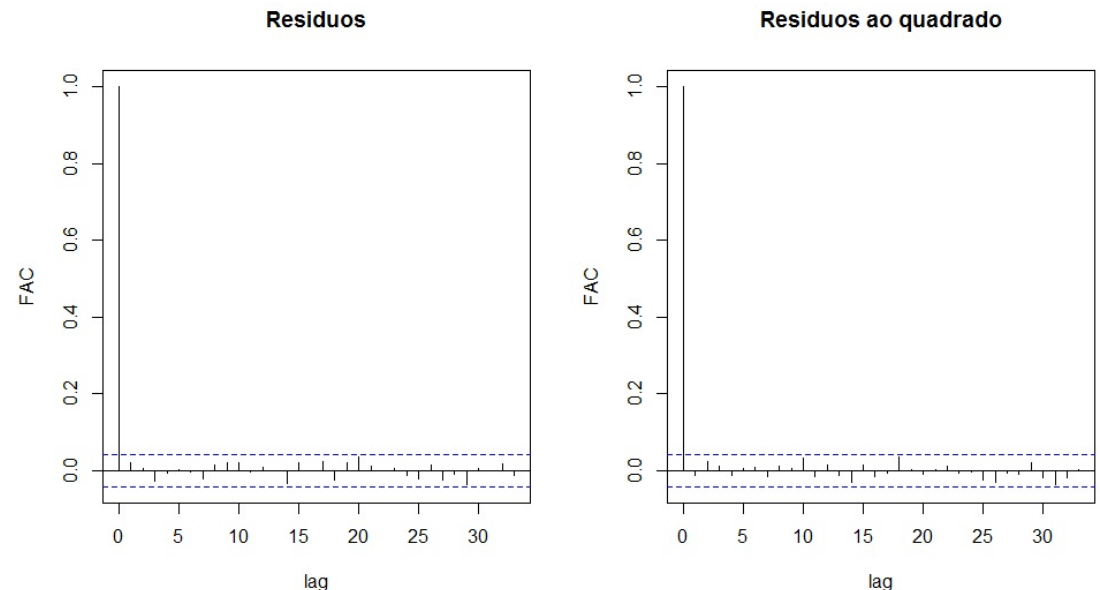

Figura 3.4: Função de autocorrelação e função de autocorrelação ao quadrado dos resíduos padronizados Modelo $\operatorname{GARH}(1,1)$ da série de retornos do Ibovespa

\subsection{Extensões dos Modelos GARCH}

Em muitos casos, o modelo GARCH básico (3.4) fornece um modelo razoavelmente bom para analisar séries temporais financeiras e para estimar a volatilidade condicional. No entanto, existem alguns aspectos do modelo que podem ser melhorados para que, assim, ocorra uma melhor captura das características e da dinâmica de uma particular série temporal.

Efeitos de Alavancagem Assimétrica e Impactos de Informações Externas

No modelo GARCH básico (3.4), uma vez que apenas os resíduos ao quadrado $\epsilon_{t-i}^{2}$ entram na equação, os sinais dos resíduos ou choques não têm efeitos na volatilidade condicional. Entretanto, 
um fato estilizado da volatilidade financeria é que más notícias (choques negativos) tendem a oferecer maior impacto na volatilidade do que boas notícias (choques positivos). Black (1976), atribui esse efeito ao fato de que más notícias tendem a baixar os preços das ações, e portanto aumentam a alavancagem da ação causando maior volatilidade nessa ação. Baseado nessa conjectura, o impacto assimétrico das notícias, das informações externas, é normalmente chamado de efeito de alavancagem ou efeito alavanca (leverage effect). Um modo de incluir esse efeito é utilizar os modelos com efeito do sinal.

Em investimentos financeiros espera-se que alto risco resulte em altos retornos. Apesar da teoria moderna de precificação de ativos ou ações não implicar uma relação simples, ela sugere que existam algumas interações entre retornos esperados e risco que são medidas pela volatilidade. Engle (1987), Lilien e Robins propuseram extender o modelo GARCH básico de modo que a volatilidade condicional pudesse gerar um prêmio de risco (risk premium), que é parte dos retornos esperados. Este modelo GARCH extendido é usualmente chamado de modelo GARCH-in-the-mean, ou modelo GARCH-M.

O modelo GARCH-M extende a equação da média condicional (3.1) conforme segue:

$$
y_{t}=c+\alpha g\left(\sigma_{t}\right)+\epsilon_{t}
$$

onde $g(\cdot)$ pode ser uma função arbitrária da volatilidade $\sigma_{t}$. Por exemplo, algumas possíveis funções $g\left(\sigma_{t}\right)$ podem ser $\sigma_{t}, \sigma_{t}^{2}$ ou $\log \left(\sigma_{t}^{2}\right)$.

\section{Variáveis Exógenas na Equação Geral da Média Condicional}

Até o momento a equação da média condicional ficou restrita a uma constante nos modelos GARCH, com exceção do modelo GARCH-M onde a volatilidade leva em conta a equação da média como uma variável explicativa.

A forma geral da média condicional é dada por

$$
y_{t}=c+\sum_{i=1}^{r} \phi_{i} y_{t-i}+\sum_{j=1}^{s} \theta_{j} \epsilon_{t-j}+\sum_{l=1}^{L} \beta_{l} x_{t-l}+\epsilon_{t}
$$

onde $x_{t}$ é um vetor $k \times 1$ de variáveis exógenas, e $\beta_{t}$ é o vetor $k \times 1$ de coeficientes.

\subsection{Distribuição de Erros Não-Gaussianos}

Até o momento, foi utilizada a suposição da distribuição normal para os erros. No entanto, é conhecido o fato de que séries temporais financeiras possuem caudas pesadas, portanto é de interesse usar distribuições que possuem caudas mais pesadas do que as da distribuição normal. Descreveremos aqui três possiveis distribuições de erros com caudas pesadas para o ajuste de modelos GARCH: a distribuição $t$-Student, a distribuição dupla e a distribuição do erro generalizado.

Se uma variável aleatória $u_{t}$ tem distribuição $t$-Student com $\nu$ graus de liberdade e com parâmetro de escala $s_{t}$, a função densidade de probabilidade de $u_{t}$ é dada por

$$
f\left(u_{t}\right)=\frac{\Gamma[(\nu+1) / 2]}{(\pi \nu)^{1 / 2} \Gamma(\nu / 2)} \frac{s_{t}^{-1 / 2}}{\left[1+\mu_{t}^{2} /\left(s_{t} \nu\right)\right]^{(\nu+1) / 2}},
$$


onde $\Gamma(\cdot)$ é a função gama. A variância de $u_{t}$ é dada por:

$$
\operatorname{Var}\left(u_{t}\right)=\frac{s_{t} \nu}{\nu-2}, \nu>2
$$

Se o termo de erro $\epsilon_{t}$ em um modelo GARCH segue uma distribuição $t$-Student com $\nu$ graus de liberdade e $\operatorname{Var}_{t-1}\left(\epsilon_{t}\right)=\sigma_{t}^{2}$, o parâmetro de escala $s_{t}$ pode ser escrito como

$$
s_{t}=\frac{\sigma_{t}^{2}(\nu-2)}{\nu}
$$

Portanto, a função de log verossimilhança de um modelo GARCH com distribuição $t$-Student para os erros pode ser facilmente construída baseada na função densidade acima.

\section{Distribuição do Erro Generalizada}

Nelson (1991) propôs o uso da distribuição do erro generalizada (GED) para capturar as caudas pesadas geralmente observadas na distribuição de séries temporais financeiras. Se a variável aleatória $u_{t}$ é uma GED com média zero e variância unitária, a função densidade de $u_{t}$ é dada por:

$$
f\left(u_{t}\right)=\frac{\nu \exp \left[-(1 / 2)\left|u_{t} / \lambda\right|^{\nu}\right]}{\lambda \cdot 2^{(\nu+1) / \nu} \Gamma(1 / \nu)}
$$

onde

$$
\lambda=\left[\frac{2^{-2 / \nu} \Gamma(1 / \nu)}{\Gamma(3 / \nu)}\right]^{1 / 2}
$$

e $\nu$ é um parâmetro positivo que determina o comportamento das caudas dessa distribuição. Quando $\nu=2$, a densidade acima se reduz a densidade normal padrão; quando $\nu<2$, a densidade possui caudas mais densas do que da distribuição normal; quando $\nu>2$, a densidade possui caudas mais leves do que a da normal.

Quando $\nu=1$, a densidade de GED se reduz à distribuição exponencial dupla:

$$
f\left(\mu_{t}\right)=\frac{1}{\sqrt{2}} \exp ^{-\sqrt{2}\left|\mu_{t}\right|} .
$$

Baseado nas funções densidade apresentadas acima, a função de log verossimilhança do modelo GARCH com GED ou de erros com distribuição exponencial dupla podem ser facilmente construídas.

\subsection{Seleção e Comparação de Modelos GARCH}

As seções anteriores ilustraram extensões dos modelos GARCH. Selecionar o melhor modelo para determinado conjunto de dados pode ser uma tarefa muito complicada. O diagnóstico de modelos baseado nos resíduos padronizados e curvas de impacto para os efeitos de alavancagem pode ser usado para comparar a eficácia de diferentes aspectos dos modelos GARCH. Além disso, uma vez que os modelos GARCH podem ser tratados como modelos ARMA para resíduos quadráticos, os critérios tradicionais de seleção de modelos como o critério de informação de Akaike (AIC) e o critério da informação Bayesiana (BIC) também podem ser usados para selecionar os melhores modelos.

Considerando o ajuste de um modelo $\operatorname{GARCH}(1,1)$ simples e um modelo com distribuição $t$-Student para a série de log-retornos do índice Bovespa, temos que o AIC e BIC do modelo com distribuição t-Student é menor do que o modelo com distribuição normal, o que sugere que a distribuição tStudent seja melhor que a distribuição normal. Os valores de BIC, AIC e log da verossimilhança 
dos modelos ajustados são apresentados na Tabela 3.3.

Tabela 3.3: $A I C$, BIC e log da verossimilhança dos modelos $G A R C H(1,1)$ ajustados para a série de retornos Ibovespa

\begin{tabular}{lll}
\hline & Distribuição Normal & Distribuição t-Student \\
\hline AIC & 3,855 & 3,851 \\
BIC & 3,865 & 3,861 \\
log-Verossimilhança & $-4418,49$ & $-4413,98$ \\
\hline
\end{tabular}

Podemos comparar graficamente os ajustes dos modelos. Na Figura 3.5 são apresentadas as FAC do quadrado dos resíduos padronizados dos modelos ajustados. Este gráfico sugere que os dois modelos são apropriados para modelar a volatilidade condicional. Podemos comparar também os gráficos QQ-plot dos resíduos padronizados, que estão apresentados na Figura 3.6. A análise gráfica reforça a conclusão obtida na análise dos critérios tradicionais de seleção de modelos, sugerindo também que a distribuição t-Student é melhor que a distribuição normal.
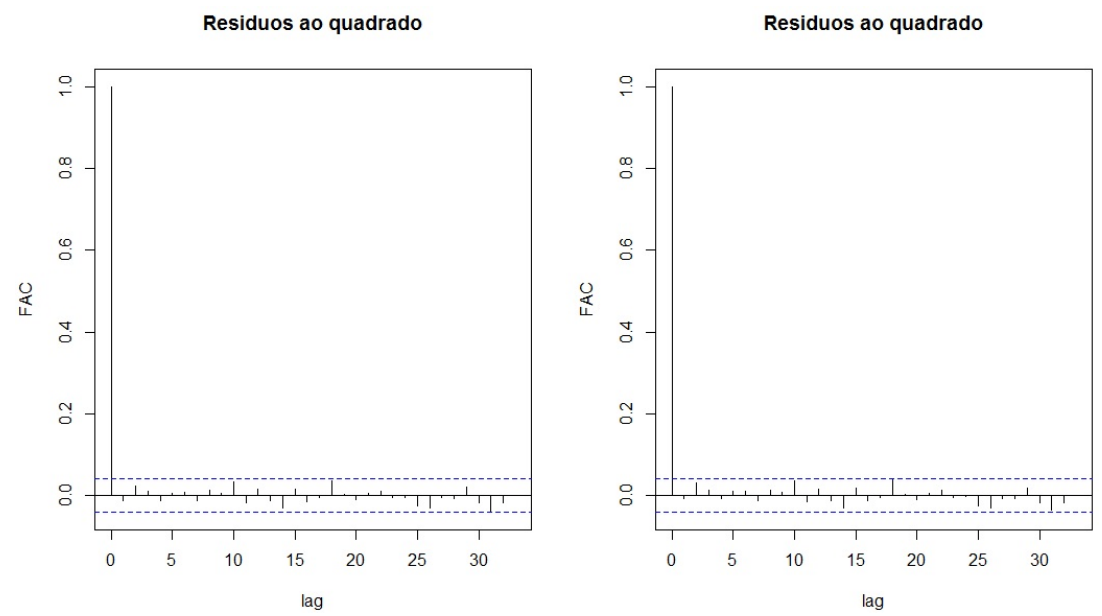

Figura 3.5: Função de autocorrelação e função de autocorrelação ao quadrado dos resíduos padronizados da Distribuição Normal e t-Student
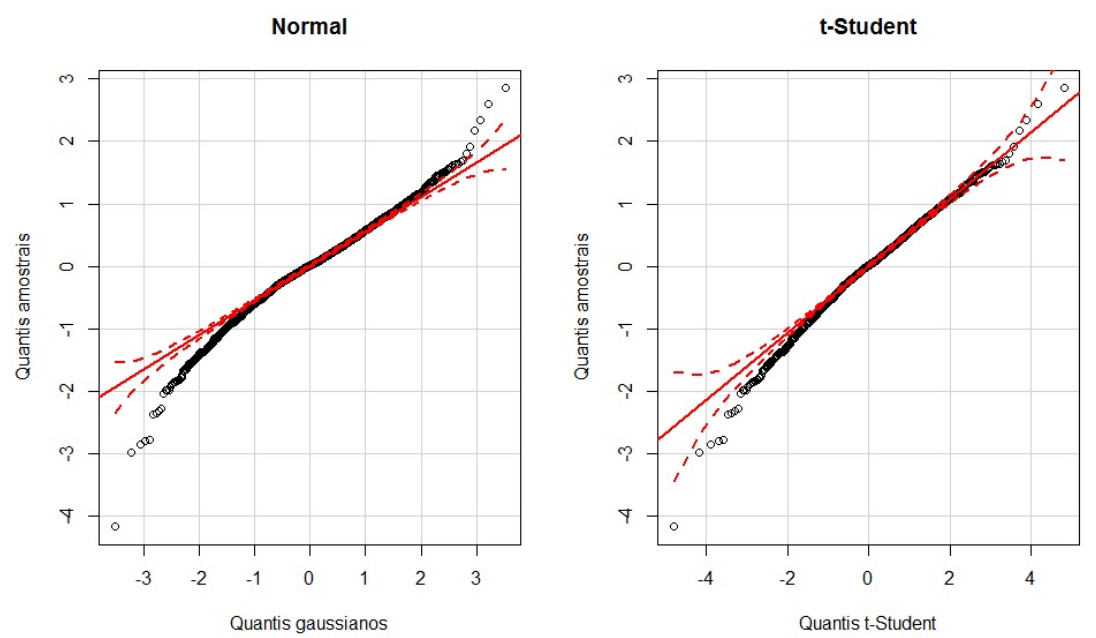

Figura 3.6: Gráfico de quantis dos Resíduos Padronizados da Distribuição Normal e t-Student 


\subsection{Predição de Modelos GARCH}

Uma tarefa importante na modelagem da volatilidade condicional é a geração de previsões precisas tanto para valores futuros de uma série temporal financeira quanto para sua volatilidade condicional. Uma vez que a média condicional do modelo geral GARCH dado em (3.17) assume a forma de um ARMA tradicional, a previsão de valores futuros de uma série temporal pode ser obtida seguindo-se a abordagem tradicional para predição de modelos ARMA. Portanto, levando-se em conta também a variância condicional variando com o tempo, modelos GARCH podem gerar previsões adequadas para os valores futuros, especialmente para horizontes pequenos. Esta seção ilustra como prever a volatilidade usando modelos GARCH.

Para simplificar, consideremos o modelo básico $\operatorname{GARCH}(1,1)$ :

$$
\sigma_{t}^{2}=a_{0}+a_{1} \epsilon_{t-1}^{2}+b_{1} \sigma_{t-1}^{2}
$$

com $t=1,2, \cdots, T$. Para obter $E_{T}\left[\sigma_{T+k}^{2}\right]$, que é a previsão da volatilidade futura $\sigma_{T+k}^{2}$, para $k>0$, dada a informação no tempo $T$, a partir da equação anterior temos:

$$
E_{T}\left[\sigma_{T+1}^{2}\right]=a_{0}+a_{1} E_{T}\left[\epsilon_{T}^{2}\right]+b_{1} E_{T}\left[\sigma_{T}^{2}\right]=a_{0}+a_{1} \epsilon_{T}^{2}+b_{1} \sigma_{T}^{2},
$$

onde $\epsilon_{T}^{2}$ e $\sigma_{T}^{2}$ são os valores obtidos depois da estimação. Para $T+2$ temos:

$$
E_{T}\left[\sigma_{T+2}^{2}\right]=a_{0}+a_{1} E_{T}\left[\epsilon_{T+1}^{2}\right]+b_{1} E_{T}\left[\sigma_{T+1}^{2}\right]=a_{0}+\left(a_{1}+b_{1}\right) E_{T}\left[\sigma_{T+1}^{2}\right],
$$

onde $E_{T}\left[\epsilon_{T+1}^{2}\right]=E_{T}\left[\sigma_{T+1}^{2}\right]$. Seguindo o mesmo raciocínio, pode-se obter a equação de previsão da volatilidade condicional:

$$
E_{T}\left[\sigma_{T+k}^{2}\right]=a_{0} \sum_{i=1}^{k-2}\left(a_{1}+b_{1}\right)^{i}+\left(a_{1}+b_{1}\right)^{k-1} E_{T}\left[\sigma_{T+1}^{2}\right],
$$

para $k \geq 2$. Para $k \rightarrow \infty$, a previsão da volatilidade em (4.32) se aproxima da variância incondicional $a_{0} /\left(1-a_{1}-b_{1}\right)$ se o processo GARCH for estacionário (ou seja, se $a_{1}+b_{1}<1$ ).

O algoritmo de predição (3.23) produz uma previsão para a variância condicional $\sigma_{T+k}^{2}$. A previsão para a volatilidade condicional, $\sigma_{T+k}$, é definida pela raiz quadrada da previsão de $\sigma_{T+k}^{2}$. 


\section{Capítulo 4}

\section{Modelo GARCH com Mudança de Regime}

\subsection{Modelo GARCH com mudança de regime Markoviano}

A característica principal dos modelos com mudança de regime é a possibilidade de alguns, ou todos, os parâmetros mudarem para cada regime (ou estado) de acordo com um processo de Markov, que é regido por uma variável de estado, denotada por $s_{t}$. A ideia é ter uma mistura de distribuições com características diferentes, dos quais o modelo extrai o valor atual da variável, de acordo com o estado (não observado), que poderia ter determinado o seu valor. A variável estado evolui de acordo com uma cadeia de Markov estacionária de primeira ordem, com probabilidade de transição dada por

$$
\operatorname{Pr}\left(s_{t}=j \mid s_{t-1}=i\right)=p_{i j},
$$

que indica a probabilidade de mudança do estado $i$ no tempo $t-1$ para o estado $j$ no instante $t$. Geralmente, estas probabilidades são escritas na matriz de transição

$$
P=\left(\begin{array}{ll}
p_{11} & p_{21} \\
p_{12} & p_{22}
\end{array}\right)
$$

considerando a existência de somente dois regimes. A probabilidade incondicional de estar no $k$ ésimo regime é dada por $P\left(s_{t}=1\right)=\pi_{1}$, em que $\pi_{1}=\left(1-p_{22}\right) /\left(2-p_{11}-p_{22}\right)$.

O modelo $\operatorname{GARCH}(1,1)$ com 2 regimes pode ser escrito na forma geral como:

$$
r_{t} \mid \zeta_{t-1}=f\left(\theta_{t}^{(i)}\right), \text { se } s_{t}=i,
$$

em que $i=1$ ou 2 e $f(\cdot)$ representa uma das possíveis densidades condicionais que podem ser assumidas normal, $t$-Student ou GED, e $\theta_{t}^{(i)}$ denota o vetor de parâmetros no $i$-ésimo regime que caracteriza a distribuição.

O vetor de parâmetros é dado da seguinte forma:

$$
\theta_{t}^{(i)}=\left(\mu_{t}^{(i)}, h_{t}^{(i)}, \nu_{t}^{(i)}\right)
$$

onde $\mu_{t}^{(i)} \equiv E\left(r_{t} \mid \zeta_{t-1}\right)$ é a média condicional ou parâmetro de locação, $h_{t}^{(i)} \equiv \operatorname{Var}\left(r_{t} \mid \zeta_{t-1}\right)$ é a variância condicional ou parâmetro de escala, $\nu_{t}^{(i)}$ é outro parâmetro da distribuição condicional e 
$\zeta_{t-1}$ é a informação até o instante $t-1$. Por isso, a família de funções de densidade de $r_{t}$ é uma família com parâmetros de locação ou escala que mudam ao longo do tempo de acordo com o regime $s_{t}=i$.

Portanto, o MRS-GARCH consiste de quatro elementos: a média condicional, a variância condicional, o parâmetro da distribuição condicional e o período de transição. A equação da média condicional, que é geralmente modelada por meio de um passeio aleatório, aqui é simplesmente modelada como

$$
r_{t}=\mu_{t}^{(i)}+\epsilon_{t}
$$

onde $i=1,2, \epsilon_{t}=\eta_{t}\left[h_{t}^{(i)}\right]^{1 / 2}$ e $\eta_{t}$ é um processo com média zero e variância unitária. A principal razão para esta escolha é o nosso foco na modelagem de volatilidade.

Uma informação que pode ser util é a duração média de cada regime dado que estamos num regime específico. (Hamilton (1989)).

Definindo $W$ como a duração num determinado regime podemos observar que:

$$
\begin{array}{ll}
w=1 ; & \text { se } \quad s_{t}=i, s_{t+1}=1, s_{t+2}=2 ; p\left(w=1 \mid s_{t}=i ; s_{t+1}=i\right)=1-p_{i i} \\
w=2 ; & \text { se } \quad s_{t}=s_{t+1}=i, s_{t+2}=i ; p\left(w=2 \mid s_{t}=s_{t+1}=i ; s_{t+2}=i\right)=p_{i i}\left(1-p_{i i}\right) .
\end{array}
$$

Observe que a variável aleatória $W$ tem distribuição geométrica e, portanto a duração esperada do regime $s_{t}=i$ com $i=1,2$ é dada por:

$$
E[w]=\frac{1}{1-p_{i i}} .
$$

\subsection{MRS-GARCH}

Recentemente, Klaassen (2002) sugere o uso da média condicional e variância condicional dos regimes do passado, levando em conta também o atual. Klaassen (2002) adota a seguinte expressão para a variância condicional

$$
h_{t}^{(i)}=\alpha_{0}^{(i)}+\alpha_{1}^{(i)} \epsilon_{t-1}^{2}+\beta_{1}^{(i)} E_{t-1}\left\{h_{t-1}^{(i)} \mid s_{t}\right\},
$$

onde a esperança condicional ao regime $s_{t}$ é

$$
E_{t-1}\left\{h_{t-1}^{(i)} \mid s_{t}\right\}=\sum_{j=1}^{2} \widetilde{p}_{i j, t-1}\left[\left(\mu_{t-1}^{(j)^{2}}+h_{t-1}^{(j)}\right)\right]-\left[\sum_{j=1}^{2} \widetilde{p}_{j i, t-1} \mu_{t-1}^{(j)}\right]^{2}
$$

e as probabilidades condicionais são dadas por

$$
\widetilde{p}_{i j, t}=\operatorname{Pr}\left(s_{t}=j \mid s_{t+1}=i, \zeta_{t-1}\right)=\frac{p_{i j} \operatorname{Pr}\left(s_{t}=j \mid \zeta_{t-1}\right)}{\operatorname{Pr}\left(s_{t+1}=i \mid \zeta_{t-1}\right)}=\frac{p_{j i} p_{j, t}}{p_{i, t+1}},
$$

onde $i, j=1,2$, e $\zeta_{t-1}$ denota a informação até o instante $t-1$.

Na literatura é usual utilizar o método de máxima verossimilhança para estimar parâmetros do modelo de mudança de regime markoviano. O essencial é obter a probabilidade a priori $p_{1, t}=$ $\operatorname{Pr}\left[s_{t}=1 \mid \zeta_{t-1}\right]$ ou seja, a probabilidade do regime 1 no tempo $t$ dada a informação no instante 
$t-1$, o qual é dada por

$$
p_{j, t}=\operatorname{Pr}\left(s_{t}=j \mid \zeta_{t-1}\right)=\sum_{i=1}^{2} p_{i j}\left[\frac{f\left(r_{t-1} \mid s_{t-1}=i\right) p_{i, t-1}}{\sum_{k=1}^{2} f\left(r_{t-1} \mid s_{t-1}=k\right) p_{k, t-1}}\right],
$$

onde $p_{i j}$ são as probabilidades de transição e $f(\cdot)$ é a densidade de probabilidade dos log-retornos. Então a função de log-verossimilhança pode ser escrita como

$$
l=\sum_{t=-R+w+1}^{T+w} \log \left[p_{1, t} f\left(r_{t} \mid s_{t}=1\right)+\left(1-p_{1, t}\right) f\left(r_{t} \mid s_{t}=2\right)\right],
$$

onde $w=0,1, \ldots, n$, e $f\left(\cdot \mid s_{t}=i\right)$ é a distribuição condicional dado que o regime $i$ ocorre no tempo $t$.

\subsection{Estimação}

Nesta seção derivamos a função de verossimilhança do modelo GARCH com mudança de regime e pode-se encontrar em Gray (1996a) que esta tem uma estrutura recursiva de primeira ordem. Seja $p_{t-1}\left(r_{t}\right)$ que denota a densidade avaliada no retorno igual a $r_{t}$. Isso pode ser expressado como

$$
p_{t-1}\left(r_{t}\right)=\sum_{s_{t}=1,2} p_{t-1}\left(r_{t} \mid s_{t}\right) \cdot p_{t-1}\left(s_{t}\right)
$$

O primeiro termo do lado direiro $p_{t-1}\left(r_{t} \mid s_{t}\right)$ denota a densidade do retorno do preço da ação até o tempo $t$ avaliado no valor $r_{t}$ condicionada em $\zeta_{t-1}$ e o regime tendo valor $s_{t}$. Esta densidade segue as formulas (4.2) e (4.3), entretanto não é simples calcular a variância condicional em (4.3). Isso requer a integração do regime $\tilde{s}_{t-1}$ em $E_{t-1}\left[\operatorname{Var}_{t-2}\left\{\epsilon_{t-1} \mid \tilde{s}_{t-1}\right\} \mid s_{t}\right]$, porque $\operatorname{Var}_{t-2}\left\{\epsilon_{t-1} \mid \tilde{s}_{t-1}\right\}$ depende somente de $s_{t-1}$ e precisamos apenas de $p_{t-1}\left(s_{t-1} \mid s_{t}\right)$, a probabilidade que o regime anterior era $s_{t-1}$ dado que o regime atual é $s_{t}$ e dada a informação $\zeta_{t-1}$ :

$$
p_{t-1}\left(s_{t-1} \mid s_{t}\right)=\frac{p_{t-1}\left(s_{t-1}\right) \cdot p_{t-1}\left(s_{t} \mid s_{t-1}\right)}{p_{t-1}\left(s_{t}\right)}
$$

onde

$$
p_{t-1}\left(s_{t}\right)=\sum_{s_{t}=1,2} p_{t-1}\left(s_{t-1}\right) \cdot p_{t-1}\left(s_{t} \mid s_{t-1}\right)
$$

A probabilidade de mudança de regime $p_{t-1}\left(s_{t} \mid s_{t-1}\right)$ segue de (4.1).

O termo restante em (4.6) e (4.7) é $p_{t-1}\left(s_{t-1}\right)$. Esta probabilidade é crucial já que todos os regimes de probabilidade deste trabalho podem ser derivados dele. Usando a tecnica similar à de Gray (1996a), a seguinte formula mostra que estas probabilidades tem uma estrutura recursiva de primeira ordem, o que simplifica substancialmente o seu cálculo:

$$
\begin{aligned}
p_{t-1}\left(s_{t-1}\right) & =p_{t-2}\left(s_{t-1} \mid r_{t-1}\right) \\
& =\frac{p_{t-2}\left(r_{t-1} \mid s_{t-1}\right) \cdot p_{t-2}\left(s_{t-1}\right)}{p_{t-2}\left(r_{t-1}\right)} \\
& =\frac{p_{t-2}\left(r_{t-1} \mid s_{t-1}\right) \cdot \sum_{s_{t-2}=1,2} p_{t-2}\left(s_{t-2}\right) \cdot p_{t-2}\left(s_{t-1} \mid s_{t-2}\right)}{p_{t-2}\left(r_{t-1}\right)}
\end{aligned}
$$

Por isso, as variáveis para calcular $p_{t-1}\left(s_{t-1}\right)$ são seus valores anteriores $p_{t-2}\left(s_{t-2}\right)$ e a constante $p_{t-2}\left(s_{t-1} \mid s_{t-2}\right)$ para $s_{t-2}=1,2$ e as densidades anteriores $p_{t-2}\left(r_{t-1} \mid s_{t-1}\right)$ e $p_{t-2}\left(s_{t-1}\right)$. Isto faz o cálculo de $p_{t-1}\left(s_{t-1}\right)$ um processo recursivo de primeira ordem. O segundo termo do lado direito de 
(4.5), $p_{t-1}\left(s_{t}\right)$, é a probabilidade condicional que o regime atual é $s_{t}$. Isso é dado por (4.7).

Tendo discutido ambos termos do lado direito de (4.5), podemos agora calcular a densidade de interesse, sendo uma distribuição normal, t-student ou GED. Estas densidades podem então ser usadas para construir a log-verossimilhança $\sum_{t=1}^{T} \log \left(p_{t-1}\left(r_{t}\right)\right)$ com o qual todos os parâmetros do modelo GARCH com mudança de regime podem ser estimados.

Do ponto de vista prático, é importante perceber que a log-verossimilhança tem uma estrutura recursiva de primeira ordem, similar ao modelo padrão, modelo $\operatorname{GARCH}(1,1) \mathrm{com}$ um regime. Depois de tudo, de (4.6) e (4.7) precisamos apenas da constante $p_{t-1}\left(s_{t} \mid s_{t-1}\right)$ e da probabilidade recursiva de primeira ordem $p_{t-1}\left(s_{t-1}\right)$ em (4.7) para todas as 4 combinações de $\left(s_{t}, s_{t-1}\right)$; então a densidade (4.5) pode ser calculada de (4.7), a mudança anterior $r_{t-1},(4.6)$ e as variâncias anteriores $V_{t-2}\left\{\epsilon_{t-1} \mid s_{t-1}\right\}$ para $s_{t-1}=1,2$. Esta recursividade de primeira ordem de $p_{t-1}\left(r_{t}\right)$ agiliza substancialmente o calculo da log-verossimilhança.

Para começar o processo recursivo, nós definimos o conjunto de variáveis igual à esperança sem o condicionamento do conjunto de informações, isto é a variância incondicional $h_{t}^{(i)}$ dada em (4.4).

\subsection{Probabilidade Suavizada}

A probabilidade suavizada que o regime é $s_{t}$ no tempo $t, p_{T}\left(s_{t}\right)$, é a probabilidade do regime $s_{t}$ dada a informação da série inteira e pode ser calculada recursivamente. Mais geralmente, qualquer probabilidade do regime anterior $p_{\tau}\left(s_{t}\right)$, dado um tempo futuro $\tau \in\{t, t+1, \ldots T\}$, pode ser calculado em uma forma recursiva, a partir da probabilidade anterior $p_{t-1}\left(s_{t}\right)$. Nesta seção será verificado esta afirmação.

Pode-se escrever $p_{\tau}\left(s_{t}\right)$ para ambos regimes $s_{t}=1,2$ conforme

$$
\begin{aligned}
p_{\tau}\left(s_{t}\right) & =p_{\tau-1}\left(s_{t} \mid r_{\tau}\right) \\
& =\frac{p_{\tau-1}\left(r_{\tau} \mid s_{t}\right) \cdot p_{\tau-1}\left(s_{t}\right)}{\sum_{s_{t}=1,2} p_{\tau-1}\left(r_{\tau} \mid s_{t}\right) \cdot p_{\tau-1}\left(s_{t}\right)} .
\end{aligned}
$$

Suponha primeiro que $\tau=t$. Então $p_{\tau}\left(s_{t}\right)$ segue diretamente, como $p_{\tau-1}\left(s_{t}\right)$ e $p_{\tau-1}\left(r_{\tau} \mid s_{t}\right)$ em (4.9) são conhecidos a partir do processo de estimação (ver seção de estimação).

Assim, vamos supor a partir de agora que $\tau>t$. O cálculo de (4.9) exige duas entradas. A primeira é a probabilidade anterior $p_{\tau-1}\left(s_{t}\right)$, que é conhecido a partir da recursão anterior para ambos os regimes. O segundo ingrediente de (4.9) é a densidade $p_{\tau-1}\left(r_{\tau} \mid s_{t}\right)$ para ambos os regimes. O seu cálculo exige uma série de etapas. Primeiramente escrevemos como

$$
p_{\tau-1}\left(r_{\tau} \mid s_{t}\right)=\sum_{s_{\tau}=1,2} p_{\tau-1}\left(r_{\tau} \mid s_{\tau}\right) \cdot p_{\tau-1}\left(s_{\tau} \mid s_{t}\right),
$$

onde usamos que a distribuição condicional de $r_{\tau}$ dado $s_{\tau}$ não depende do regime anterior $s_{t}$. Esta fórmula em si tem dois ingredientes. O primeiro é a densidade $p_{\tau-1}\left(r_{\tau} \mid s_{\tau}\right)$ para ambas combinações do regime, que é conhecida do processo de estimação. O segundo termo necessário em (4.10) é o $(\tau-t)$ período à frente para a probabilidade de mudança de regime $p_{\tau-1}\left(s_{\tau} \mid s_{t}\right)$ para todos os resultados do regime. Uma vez que tenha sido calculado, ele deve ser salvo, já que será necessário em uma próxima fase recursiva. Fazendo uso da estrutura do processo de regime Markov, ele pode ser escrito em termos de $(\tau-1-t)$ probabilidade de mudança em um período à frente

$$
p_{\tau-1}\left(s_{\tau} \mid s_{t}\right)=\sum_{s_{\tau-1}=1,2} p_{\tau-1}\left(s_{\tau} \mid s_{\tau-1}\right) \cdot p_{\tau-1}\left(s_{\tau-1} \mid s_{t}\right) .
$$


Mais uma vez, há dois ingredientes. Primeiro, precisamos $p_{\tau-1}\left(s_{\tau} \mid s_{\tau-1}\right)$ para todas as combinações do regime. Estes são constantes e seguem a partir de (4.1).

O segundo ingrediente de (4.11) é $p_{\tau-1}\left(s_{\tau-1} \mid s_{\tau}\right)$ para todas as combinações do regime. Temos:

$$
\begin{aligned}
p_{\tau-1}\left(s_{\tau-1} \mid s_{t}\right) & =p_{\tau-2}\left(s_{\tau-1} \mid s_{t}, r_{\tau-1}\right) \\
& =\frac{p_{\tau-2}\left(r_{\tau-1} \mid s_{\tau-1}\right) \cdot p_{\tau-2}\left(s_{\tau-1} \mid s_{t}\right)}{\sum_{s_{\tau-1}=1,2} p_{\tau-2}\left(r_{\tau-1} \mid s_{\tau-1}\right) \cdot p_{\tau-2}\left(s_{\tau-1} \mid s_{t}\right)}
\end{aligned}
$$

onde usamos essa densidade condicional de $r_{\tau-1}$ é independente do regime anterior $s_{t}$ uma vez que $s_{\tau-1}$ é dado. Temos dois ingredientes. Primeiro, a densidade condicional $p_{\tau-2}\left(r_{\tau-1} \mid s_{\tau-1}\right)$ para ambos resultados do regime. É conhecida a partir do processo de estimação. Em segundo lugar, precisamos de $(\tau-1-t)$ probabilidade de mudança em um período a frente $p_{\tau-2}\left(s_{\tau-1} \mid s_{t}\right)$ para todas as combinações do regime. Este foi salvo durante a recursão anterior, se $\tau>t+1$. Se $\tau=t+1$, isto é igual a um. Isto completa o algoritmo para calcular (4.10), que é o segundo ingrediente de (4.9). Para cada uma recursividade tem de calcular (4.12), utilizar o resultado para calcular (4.11) e usar esta para calcular (4.10). Usando isso em (4.9) produz a probabilidade posterior $p_{\tau}\left(s_{t}\right)$ de $p_{\tau-1}\left(s_{t}\right)$. Portanto, a partir da probabilidade anterior $p_{t-1}\left(s_{t}\right)$ pode-se recursivamente calcular a probabilidade posterior $p_{\tau}\left(s_{t}\right)$. 


\section{Capítulo 5}

\section{Aplicação em Séries Reais}

\subsection{Aplicações}

Neste capítulo serão apresentadas algumas aplicações a dados reais dos modelos apresentados nos capítulos anteriores. As aplicações foram realizadas em séries de log-retornos dos índices de duas grandes bolsas de valores, Ibovespa e S\&P500, obtidos no site yahoo finanças. Chamaremos os log-retornos simplesmente de retornos. O software utilizado para o ajuste do modelo com mudança de regime foi o Matlab e as outras análises foram realizadas no R-project. Ver Zivot (2003) para mais detalhes sobre o uso deste software no estudo de séries financeiras.

\subsection{IBOVESPA}

O conjunto de dados analisado neste trabalho é o índice do mercado de ações de São Paulo IBOVESPA. O período considerado é de 2 de janeiro de 2003 a 9 de abril de 2012 para um total de 2294 observações. Os dados foram obtidos a partir do site yahoo finanças. A cotação estudada foi o preço de fechamento diário do índice.

Primeiramente, apresentamos nas Figuras 5.1 e 5.2, a série Ibovespa, a série de retornos, gráfico de quantis e o histograma dos retornos.
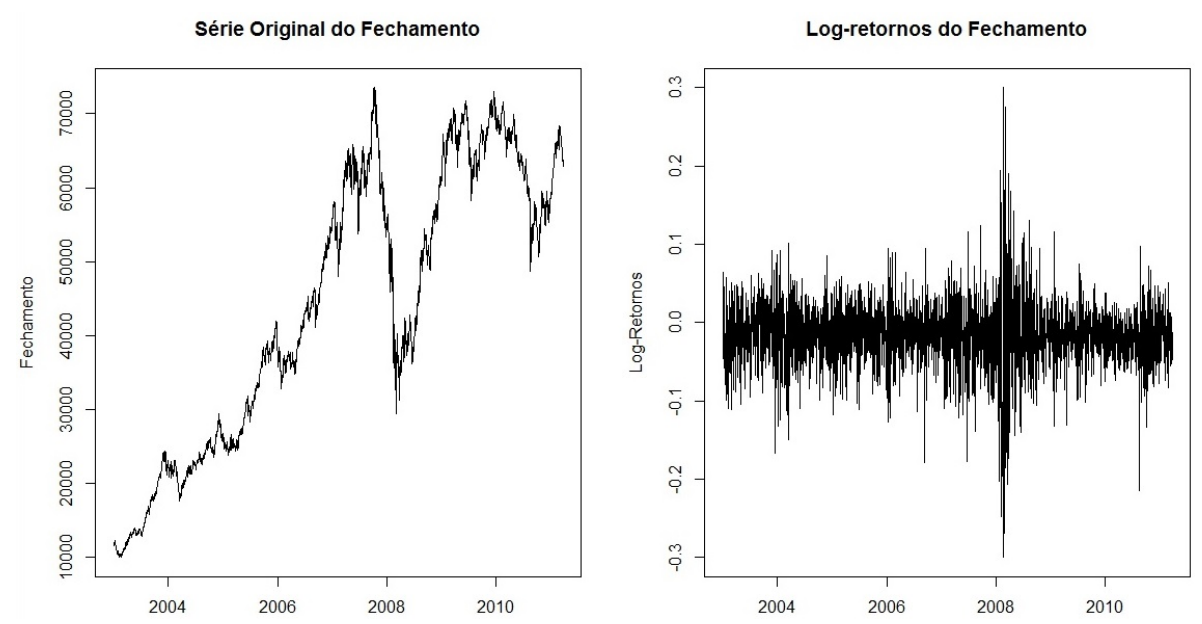

Figura 5.1: Gráfico da Série do índice Bovespa e os Log-retornos.

A Tabela 5.1 mostra algumas estatísticas descritivas dos log-retornos do Índice Bovespa. Note que a média dos dados está próxima de zero e o desvio padrão é pequeno, mas os log-retornos variam de -0.12 a 0.13 . A média é próxima de zero e, com o desvio padrão pequeno, podemos concluir que os dados se encontram aglomerados em torno do zero, o que pode ser confirmado pelo histograma dos retornos diários do IBOVESPA. Observando o gráfico da série diária do IBOVESPA, podemos ver a que a série é não estacionária, pois apresenta tendência não constante e variabilidade 

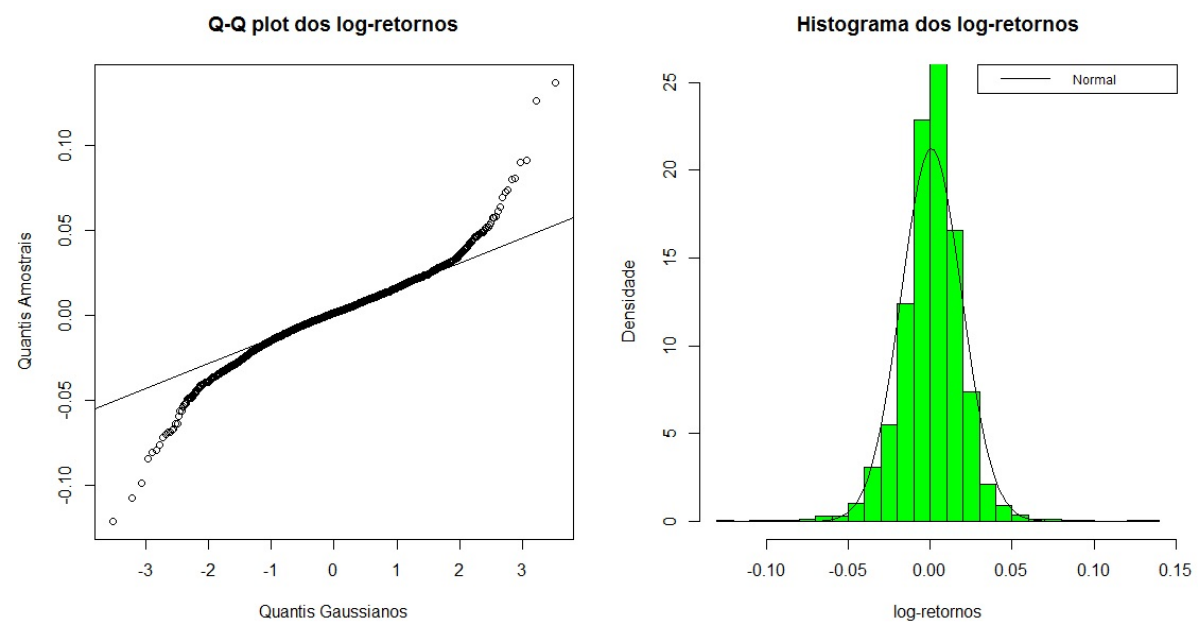

Figura 5.2: Gráfico de quantis e Histograma dos log-retornos da Série do índice Bovespa.

Tabela 5.1: Medidas descritivas do retorno-Ibovespa

\begin{tabular}{lllllll}
\hline Média & Mediana & D.Padrão & Curtose & Mínimo & Máximo & Assimetria \\
\hline 0.00073 & 0.00144 & 0.01876 & 8.20814 & -0.12100 & 0.136800 & -0.11304 \\
\hline
\end{tabular}

não controlada. Já o gráfico dos retornos diários do IBOVESPA, apresenta nível, aparentemente, constante e variabilidade controlada, o que nos leva a pensar que esta série pode ser estacionária. A assimetria e a curtose indicam que os dados não seguem uma distribuição normal. Com base nessas medidas resumo dos retornos diários da IBOVESPA, podemos observar que: a distribuição dos retornos possui uma leve assimetria à esquerda, já que o valor do coeficiente de assimetria é negativo, a medida de curtose da distribuição dos retornos indica que sua curva é mais achatada que a curva da distribuição Normal, ou seja, há uma maior quantidade de dados nas caudas da distribuição dos retornos do que em uma distribuição Normal. A assimetria e a curtose podem ser visualizadas no gráfico de quantis e histograma da Figura 5.2.

Entretanto, na Figura 5.3 observa-se que as autocorrelações dos retornos são não significativas e as autocorrelações dos retornos ao quadrado são significativas. Conforme descrito no Capítulo 3, este comportamento indica que a série de retornos pode mostrar presença de heterocedasticidade condicional nos dados, una vez que os retornos ao quadrado medem o momento de segunda ordem da série original, este resultado indica que a variância dos retornos da série do Ibovespa condicionada ao passado histórico pode sofrer alterações ao longo do tempo.

As estimativas dos parâmetros do modelo MRS-GARCH são apresentadas na Tabela 5.2. Foram ajustados os modelos com distribuição Normal, t de Student e a distribuição generalizada do erro. Para o modelo com distribuição t-Student foi considerado o modelo em que o grau de liberdade é o mesmo nos 2 regimes (t) e com graus de liberdade que podem ser diferentes (t2). Note que as estimativas e os erros padrão são bem semelhantes para os modelos com distribuição t-Student. Em particular, a Tabela 5.2 mostra o desvio padrão condicional dos retornos para cada regime da volatilidade, isto é

$$
\sigma^{(i)}=\left(a_{0}^{(i)} /\left(1-a_{1}^{(i)}-b_{1}^{(i)}\right)\right)^{1 / 2}
$$

Para os modelos t de Student, os parâmetros da média parecem negativos para o regime 1 e positivos para o regime 2. Isso parece ser o contrário para o modelo normal e para o modelo GED, parece que a média é nula para o regime 1 e positiva para o regime 2 . O parâmetro $a_{1}$, que mede o efeito do erro anterior na variância, parece não significativo nos modelos normal e t de Student. 


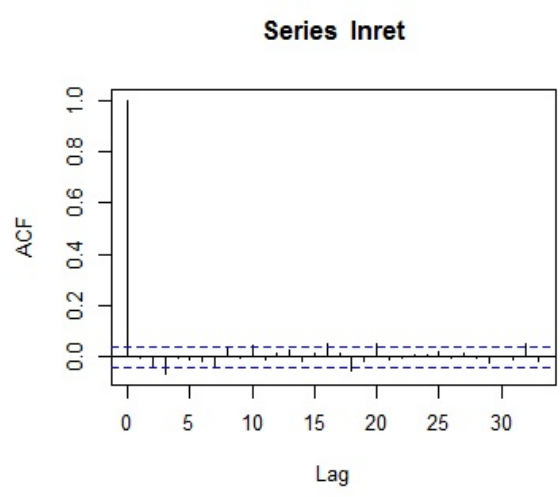

Series Inret2

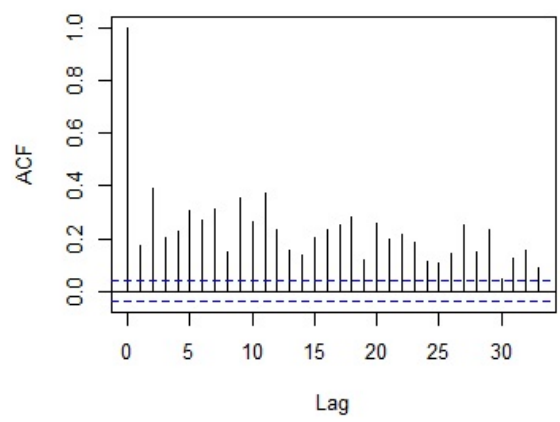

Series Inret

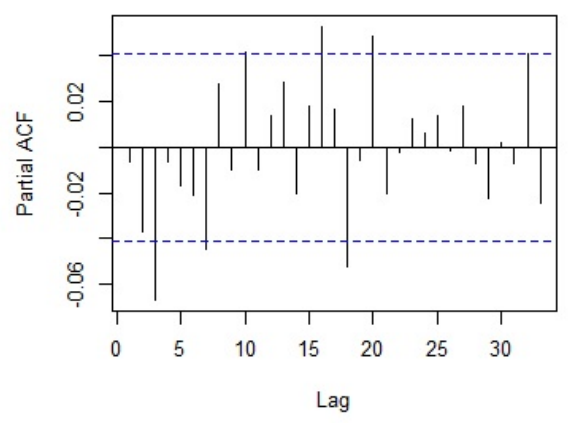

Series Inret2

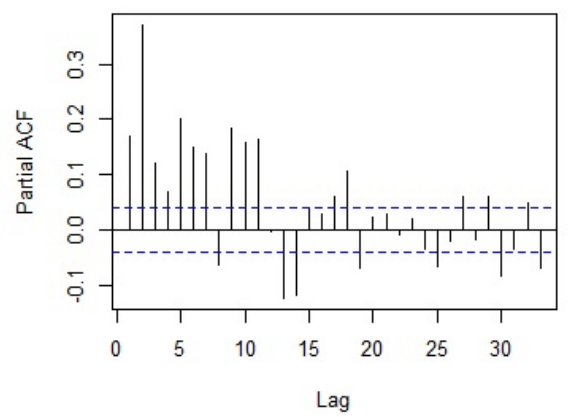

Figura 5.3: Função de autocorrelação dos log-retornos e log-retornos ao quadrado-IBOVESPA.

Para o regime 1, a estimativa é quase zero para o modelo normal e está em torno de 0.16 para os modelos t de Student. Entretanto, os valores para os parâmetros $b_{1}$ são menores no regime 1, isso significa que temos mais peso para cada erro de ontem e menor inércia para a variância anterior $\sigma_{t-1}^{2}$. A probabilidade de se manter no regime $1, p_{11}$ é menor (38\% para a distribuição t), que a probabilidade de se manter no regime 2, o mesmo acontece para a distribuição t de Student com 2 graus de liberdade.

A Tabela 5.2 também mostra as probabilidades incondicionais de cada modelo MRS-GARCH. A probabilidade incondicional $\pi_{1}$ de estar no primeiro regime varia entre $10 \%$ para o modelo t de Student e 66\% para o modelo normal. Para o modelo $t$-Student com 10 e 12 graus de liberdade no primeiro regime, o parâmetro $\nu$ indica que a distribuição tem caudas mais pesadas do que a normal. Já para o segundo regime, o valor do parâmetro $\nu$ indica que a distribuição é aproximadamente normal. No caso do GED o valor do parâmetro $\nu$ está indicando que a distribuição tem caudas mais pesadas do que a normal.

A identificação de regime com caudas mais pesadas no regime 2 da distribuição t de Student nos leva a escolher o modelo t2. O modelo MRS-GARCH com distribuição $t$-Student em que os graus de liberdade mudam em cada regime implica que a curtose varia como em Hansen (1994) e Dueker (1997). No entanto existe uma diferença entre os trabalhos mencionados. Hansen (1994) sugere um modelo no qual o parâmetro $\nu$ varia ao longo do tempo de acordo com uma função de distribuição logística no tempo $t$-1, e Dueker (1997) mostra como um parâmetro depende apenas do regime, no nosso trabalho este parâmetro só depende do regime, juntamente com todos os restantes parâmetros. 
Tabela 5.2: Estimativas de Máxima Verossimilhança erros padrões do Modelo MRS - GARCH - Ibovespa

\begin{tabular}{|c|c|c|c|c|}
\hline & MRS-GARCH-N & MRS-GARCH-t2 & MRS-GARCH- $t$ & MRS-GARCH-GED \\
\hline \multirow[t]{2}{*}{$\mu^{(1)}$} & 0.2605 & -1.2336 & -1.2622 & 0.0332 \\
\hline & $(0.0468)$ & $(0.4775)$ & $(0.5895)$ & $(0.0285)$ \\
\hline \multirow[t]{2}{*}{$\mu^{(2)}$} & -0.3768 & 0.2425 & 0.2300 & 0.0419 \\
\hline & $(0.1302)$ & $(0.0652)$ & $(0.0651)$ & $(0.0137)$ \\
\hline \multirow[t]{2}{*}{$\sigma^{(1)}$} & 0.1033 & $<0.0001$ & 0.0851 & 0.1332 \\
\hline & $(0.0531)$ & $(0.4204)$ & $(0.6107)$ & $(0.0381)$ \\
\hline \multirow[t]{2}{*}{$\sigma^{(2)}$} & 0.4053 & $<0.0001$ & $<0.0001$ & 0.0029 \\
\hline & $(0.1047)$ & $(0.0572)$ & $(0.0656)$ & $(0.0012)$ \\
\hline \multirow[t]{2}{*}{$a_{1}^{(1)}$} & $<0.0001$ & 0.1768 & 0.1546 & 0.1002 \\
\hline & $(0.0273)$ & $(0.1275)$ & $(0.1276)$ & $(0.0220)$ \\
\hline \multirow[t]{2}{*}{$a_{1}^{(2)}$} & 0.0645 & 0.0528 & 0.0520 & 0.0142 \\
\hline & $(0.0262)$ & $(0.0184)$ & $(0.0184)$ & $(0.0052)$ \\
\hline \multirow[t]{2}{*}{$b_{1}^{(1)}$} & 0.8457 & 0.8111 & 0.8332 & 0.8278 \\
\hline & $(0.0318)$ & $(0.1712)$ & $(0.1701)$ & $(0.0330)$ \\
\hline \multirow[t]{2}{*}{$b_{1}^{(2)}$} & 0.9315 & 0.8797 & 0.8805 & 0.9785 \\
\hline & $(0.0361)$ & $(0.0272)$ & $(0.0277)$ & $(0.0060)$ \\
\hline \multirow[t]{2}{*}{$p_{11}$} & 0.9107 & 0.3817 & 0.3799 & 0.9987 \\
\hline & $(0.0285)$ & $(0.1943)$ & $(0.1988)$ & $(0.0011)$ \\
\hline \multirow[t]{2}{*}{$p_{22}$} & 0.8197 & 0.9243 & 0.9304 & 0.9989 \\
\hline & $(0.0476)$ & $(0.0456)$ & $(0.0466)$ & $(<0.0001)$ \\
\hline \multirow[t]{2}{*}{$\nu^{(1)}$} & - & 10.2401 & 12.1334 & 1.2667 \\
\hline & & $(2.2062)$ & $(3.3496)$ & $(0.0309)$ \\
\hline \multirow[t]{2}{*}{$\nu^{(2)}$} & - & 81.3425 & - & - \\
\hline & & $(<0.0001)$ & & \\
\hline $1000 * \log (L)$ & -4.3928 & -4.3861 & -4.3867 & -4.3877 \\
\hline$\pi_{1}$ & 0.6687 & 0.1091 & 0.1009 & 0.4583 \\
\hline$\pi_{2}$ & 0.3313 & 0.8909 & 0.8991 & 0.5417 \\
\hline$\rho_{1}$ & 0.8457 & 0.9879 & 0.9878 & 0.9280 \\
\hline$\rho_{2}$ & 0.9960 & 0.9325 & 0.9325 & 0.9927 \\
\hline
\end{tabular}


As probabilidades suavizadas do regime 1 com erro gaussiano e os log-retornos podem ser observados no seguinte gráfico:

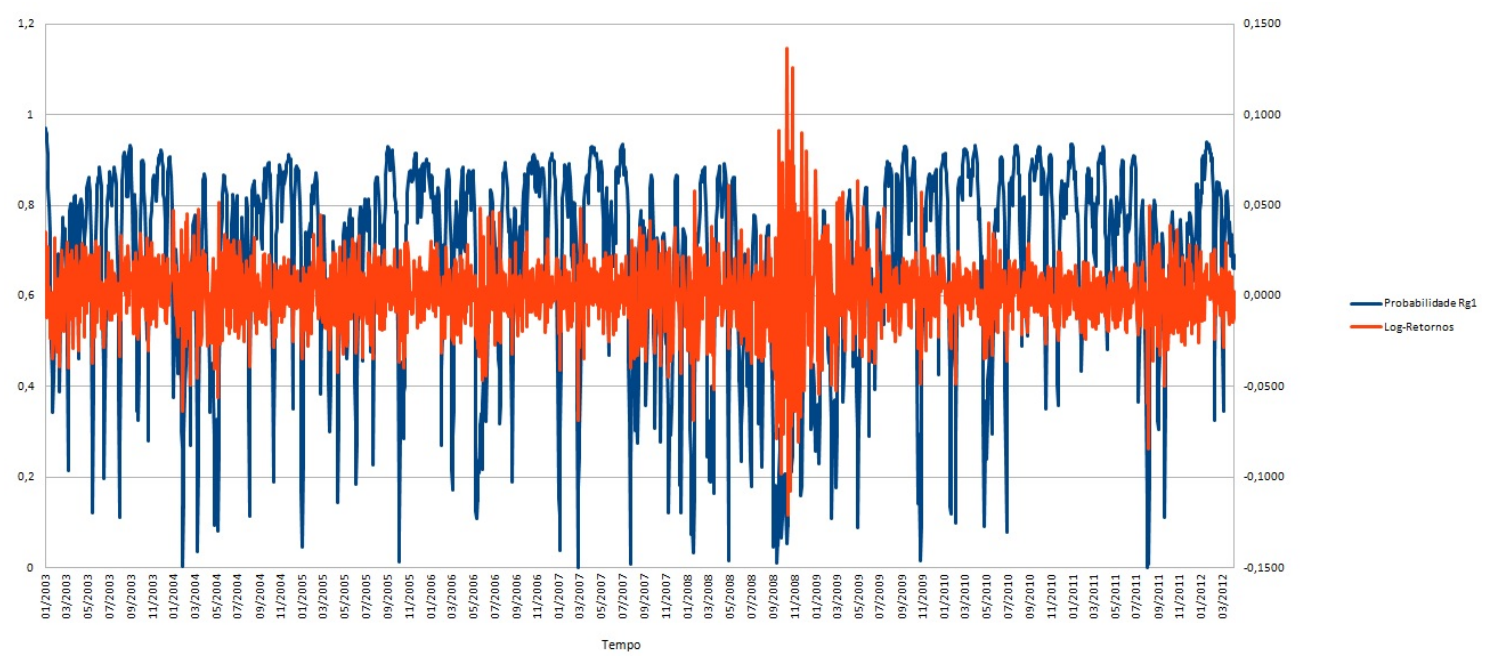

Figura 5.4: Probabilidade suavizada do Regime 1 - Ibovespa - Normal.

Em nossa aplicação existem dois regimes, $s_{t}=1$ e $s_{t}=2$, ou seja, se o processo estiver no regime 1, a observação será retirada de uma distribuição normal com média 0.2605 e desvio padrão 0.1033 e se estiver no segundo regime com média -0.3768 e desvio padrão 0.4053 . A Figura 5.4 apresenta a probabilidade suavizada do regime 1 e os log-retornos. As probabilidades de estarmos no regime 1 , variam entre 0 e 0.9 e as probabilidades de estarmos em um regime e continuarmos neste são $p_{11}$ e $p_{22}$ iguais a 0.9107 e 0.8197 respetivamente. Com isto temos que a matriz de transição é dada por

$$
P=\left(\begin{array}{ll}
0.9107 & 0.1803 \\
0.0893 & 0.8197
\end{array}\right)
$$

Temos então que a probabilidade incondicional de estar no $k$-ésimo regime, neste caso para nosso modelo com 2 regimes é dada por $\pi_{1}=0.6687$ e $\pi_{2}=0.3313$. Ou seja, ao sortearmos uma observação qualquer da série, a probabilidade é maior de estarmos no regime 1 do que no regime 2.

Uma informação que pode ser util é a duração média de cada regime dado que estamos num regime específico. (Hamilton (1989)).

Definindo $W$ como a duração num determinado regime podemos observar que:

$$
\begin{array}{ll}
w=1 ; & \text { se } \quad s_{t}=i, s_{t+1}=1, s_{t+2}=2 ; p\left(w=1 \mid s_{t}=i ; s_{t+1}=i\right)=1-p_{i i} \\
w=2 ; & \text { se } \quad s_{t}=s_{t+1}=i, s_{t+2}=i ; p\left(w=2 \mid s_{t}=s_{t+1}=i ; s_{t+2}=i\right)=p_{i i}\left(1-p_{i i}\right) .
\end{array}
$$

Observe que a variável aleatória $W$ tem distribuição geométrica e, portanto a duração esperada do regime $s_{t}=i$ com $i=1,2$ é dada por:

$$
E[w]=\frac{1}{1-p_{i i}}
$$

Logo a duração média de estarmos no regime 1 é

$$
p_{11}=\frac{1}{1-p_{11}}=\frac{1}{1-0.9107}=11 \text { dias. }
$$

E para o regime 2 é

$$
p_{22}=\frac{1}{1-p_{22}}=\frac{1}{1-0.8197}=5 \quad \text { dias. }
$$


Ao observarmos o desvio padrão condicional estimado pelo modelo com distribuição normal para a série IBOVESPA na Figura 5.5, observa-se que a volatilidade aumentou bastante durante a crise do subprime de 2008 em ambos os regimes.
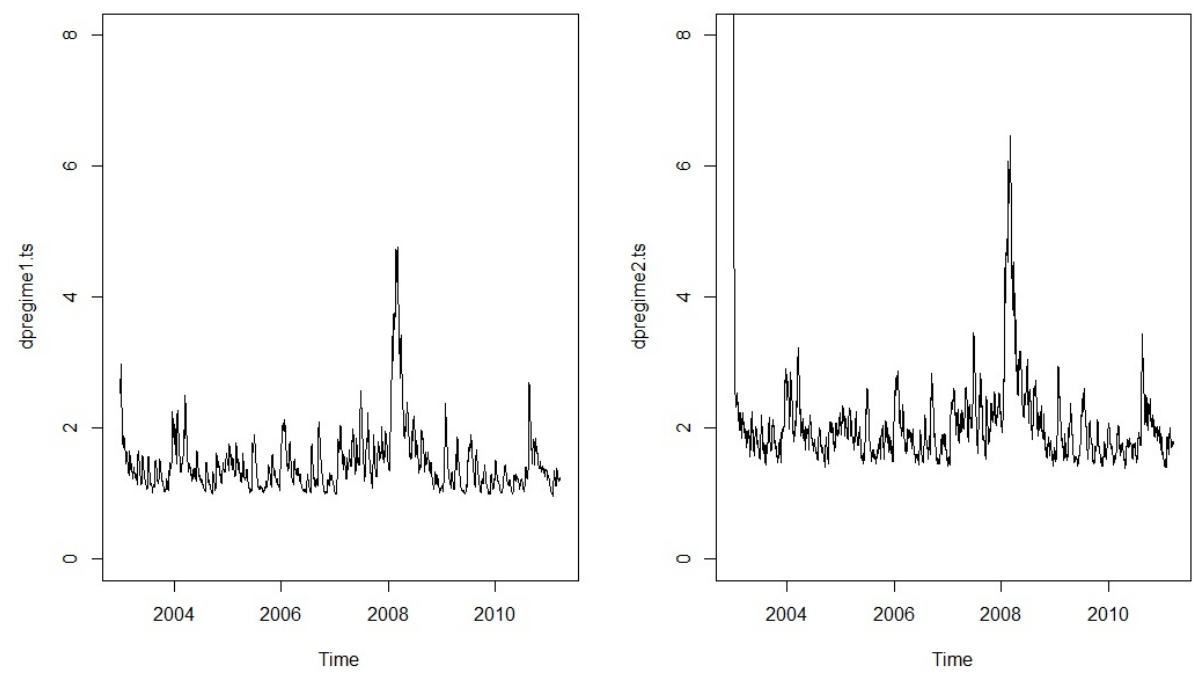

Figura 5.5: Estimativa do desvio padrão condicional para os retornos do Ibovespa no Regime 1 e 2 com erro gaussiano.

As probabilidades suavizadas do regime 1 com erro t de Student e os log-retornos podem ser observados no seguinte gráfico:

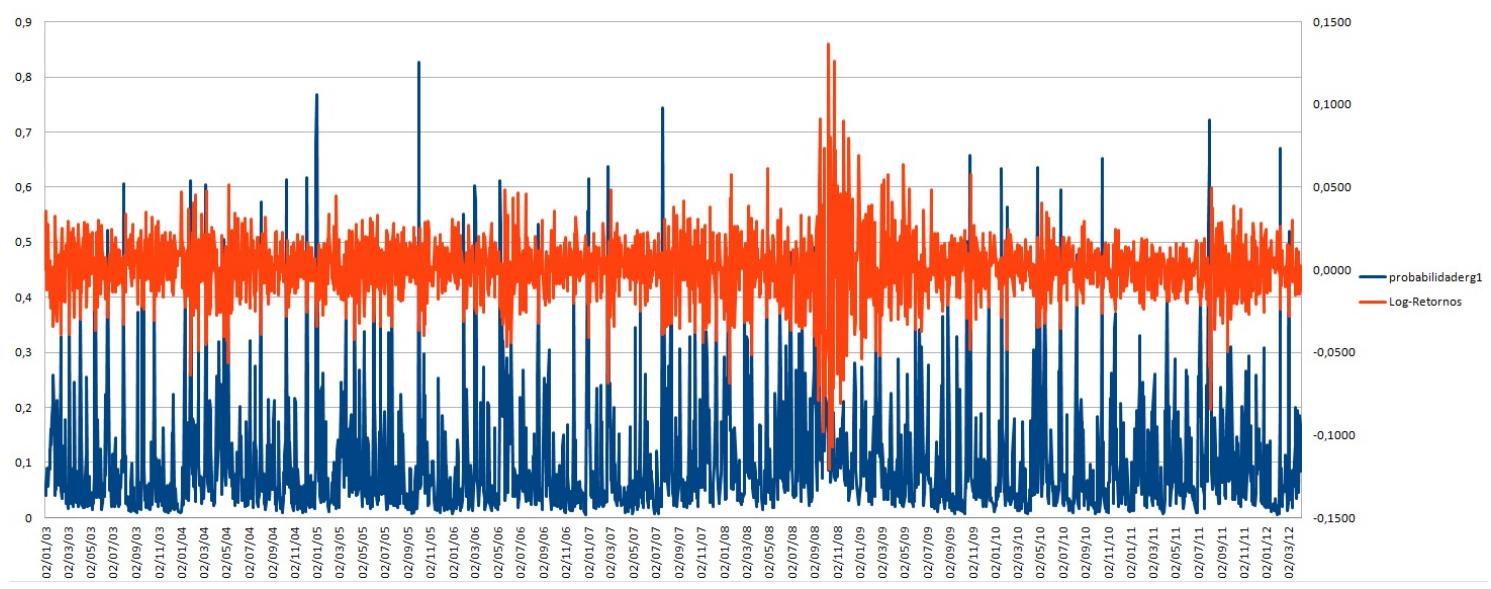

Figura 5.6: Probabilidade suavizada do Regime 1 - Ibovespa - $t$.

Se o processo estiver no regime 1, a observação será retirada de uma distribuição t de Student com média -1.2622 e desvio padrão 0.0651 e se estiver no segundo regime com média 0.2300 e desvio padrão $<0.0001$. A Figura 5.6 apresenta a probabilidade suavizada do regime 1 e os log-retornos. As probabilidades de estarmos no regime 1, variam entre 0 e 0.4 e as probabilidade de estarmos em um regime e continuarmos neste $p_{11}$ e $p_{22}$ são 0.3799 e 0.9304 . Com isto temos que a matriz de transição é dada por

$$
P=\left(\begin{array}{ll}
0.3799 & 0.0696 \\
0.6201 & 0.9304
\end{array}\right) .
$$

Temos então que a probabilidade incondicional de estar no $k$-ésimo regime, neste caso para nosso modelo com 2 regimes é dada por $\pi_{1}=0.1009$ e $\pi_{2}=0.8991$. Ou seja, ao sortearmos uma observação qualquer da série, a probabilidade é maior de estarmos no regime 2 do que no regime 1.

A duração média de estarmos no regime 1 é 1 dia e do regime 2 é de 14 dias. 

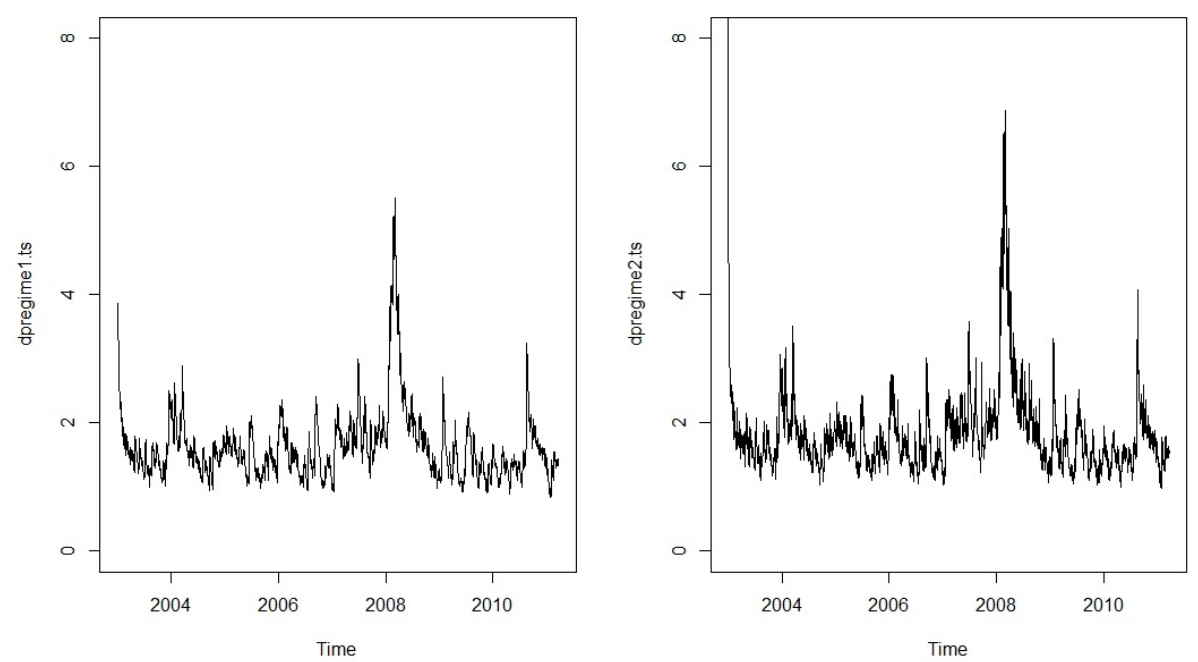

Figura 5.7: Estimativa do desvio padrão condicional para os retornos do Ibovespa no Regime 1 e 2 com erro $t$ de Student.

As probabilidades suavizadas do regime 1 com erro t de Student com distintos graus de liberdade e os log-retornos podem ser observados na Figura 5.8:

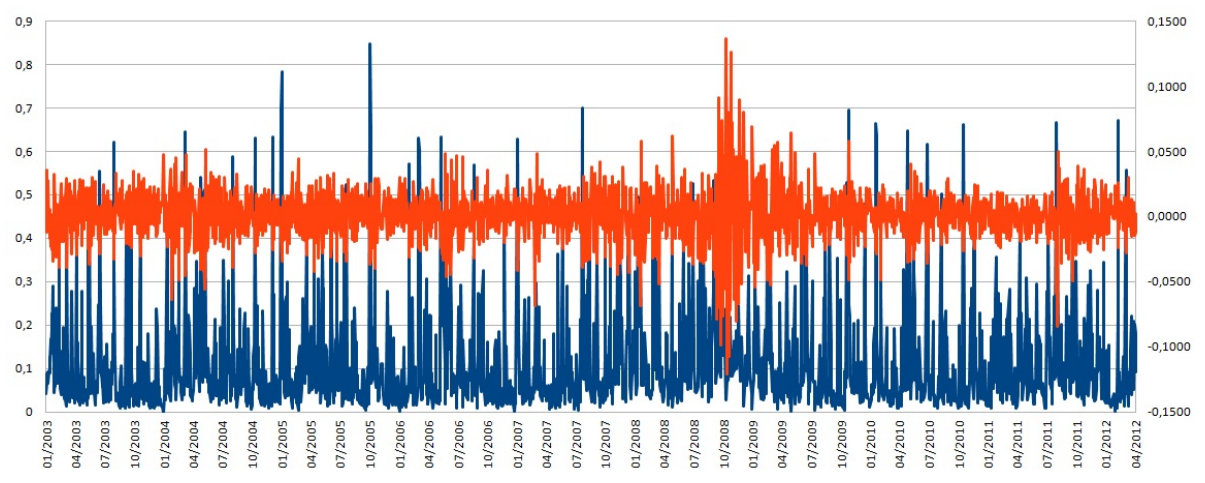

Figura 5.8: Probabilidade suavizada do Regime 1 - Ibovespa - t2.

Se o processo estiver no regime 1, a observação será retirada de uma distribuição t de Student com média -1.2336 e desvio padrão $<0.0001$ e se estiver no segundo regime com média 0.2425 e desvio padrão $<0.0001$. As probabilidades de estarmos no regime 1 , variam entre 0 e 0.38 e as probabilidades de estarmos em um regime e continuarmos neste $p_{11}$ e $p_{22}$ são 0.3817 e 0.9243 . Com isto temos que a matriz de transição é dada por

$$
P=\left(\begin{array}{ll}
0.3817 & 0.0757 \\
0.6183 & 0.9243
\end{array}\right)
$$

Temos então que a probabilidade incondicional de estar no $k$-ésimo regime, neste caso para nosso modelo com 2 regimes é dada por $\pi_{1}=0.1091$ e $\pi_{2}=0.8909$. Ou seja, temos ao sortearmos uma observação qualquer da série, a probabilidade é maior de estarmos no regime 2 do que no regime 1.

A duração média de estarmos no regime 1 é 1 dia e do regime 2 é de 13 dias. 

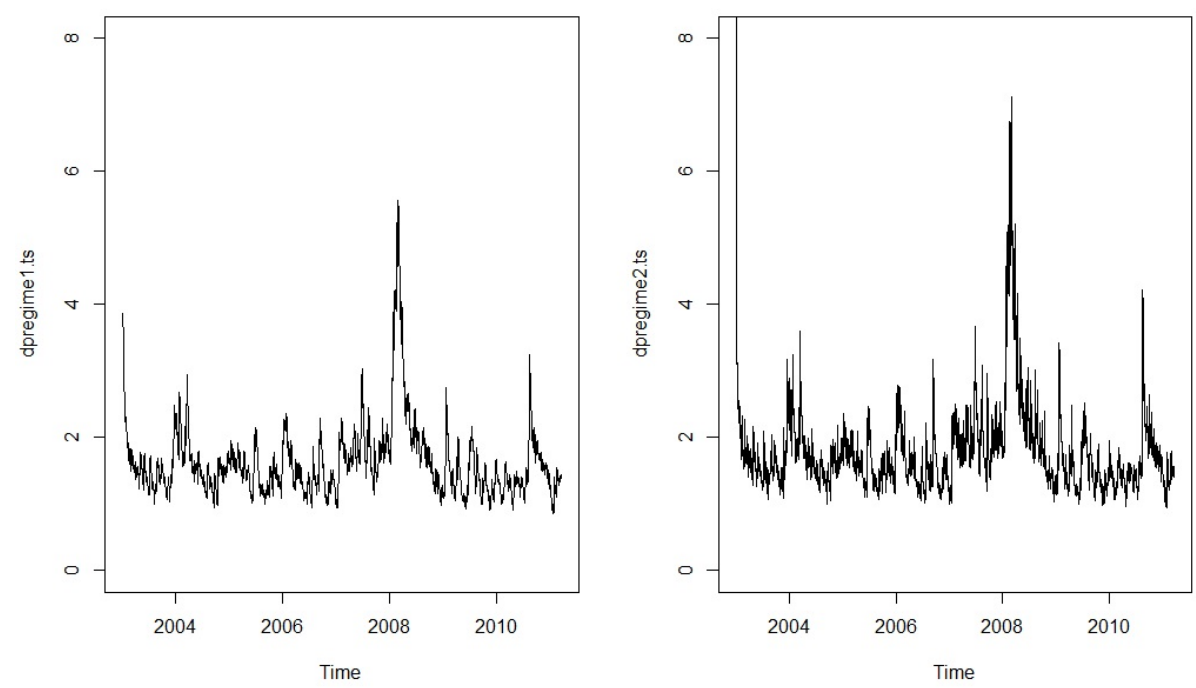

Figura 5.9: Estimativa do desvio padrão condicional para os retornos do Ibovespa no Regime 1 e 2 com erro $t$ de Student-t2.

As probabilidades suavizadas do regime 1 com erro GED e os log-retornos podem ser observados no seguinte gráfico:
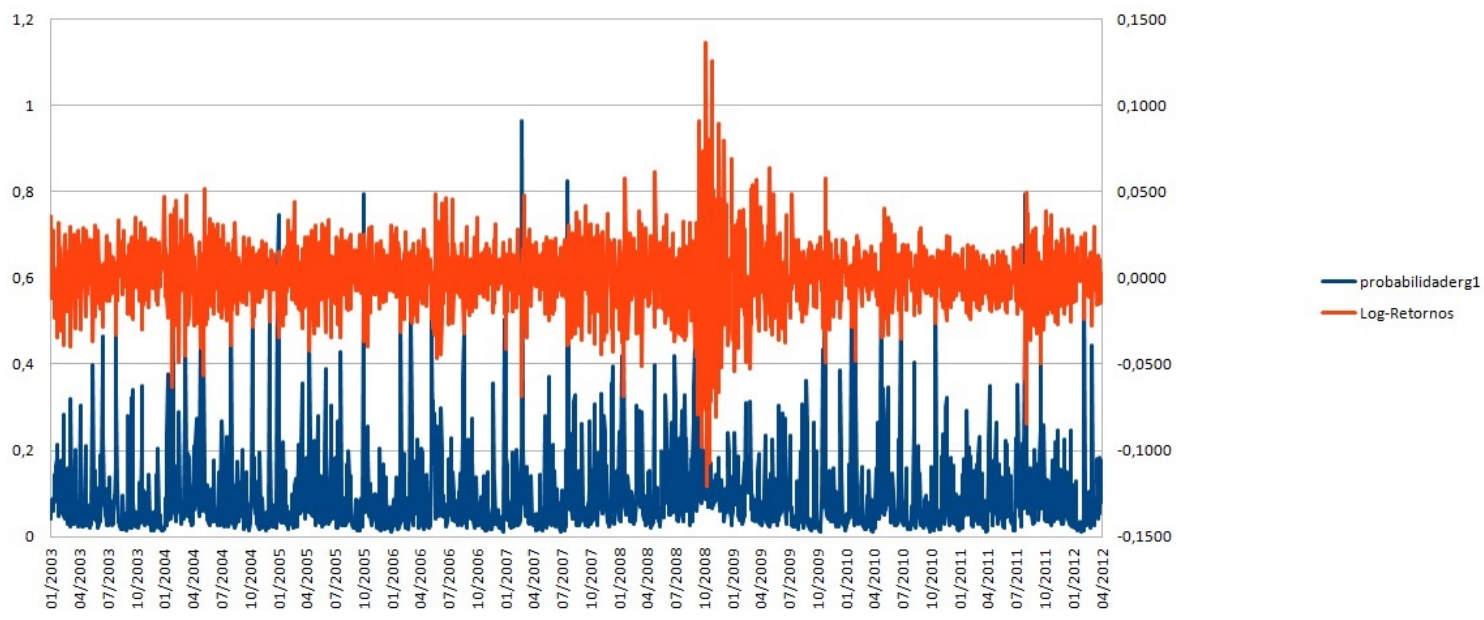

Figura 5.10: Probabilidade suavizada do Regime 1 - Ibovespa - GED.

Se o processo estiver no regime 1, a observação será retirada de uma distribuição ged com média 0.0332 e desvio padrão 0.1332 e se estiver no segundo regime com média 0.0419 e desvio padrão 0.0029 . A figura 5.10 apresenta a probabilidade suavizada do regime 1 e os log-retornos. As probabilidades de estarmos no regime 1 , variam entre 0 e 0.9 e as probabilidades de estarmos em um regime e continuarmos neste $p_{11}$ e $p_{22}$ são 0.9987 e 0.9989 respetivamente, ou seja, os estados seriam absorventes. Com isto temos que a matriz de transição e dada por

$$
P=\left(\begin{array}{ll}
0.9987 & 0.0011 \\
0.0013 & 0.9989
\end{array}\right) \text {. }
$$

Temos então que a probabilidade incondicional de estar no $k$-ésimo regime, neste caso para nosso modelo com 2 regimes é dada por $\pi_{1}=0.4583$ e $\pi_{2}=0.5417$. Ou seja, ao sortearmos uma observação qualquer da série, a probabilidade é só um pouco maior de estarmos no regime 2 do que no regime 1. 

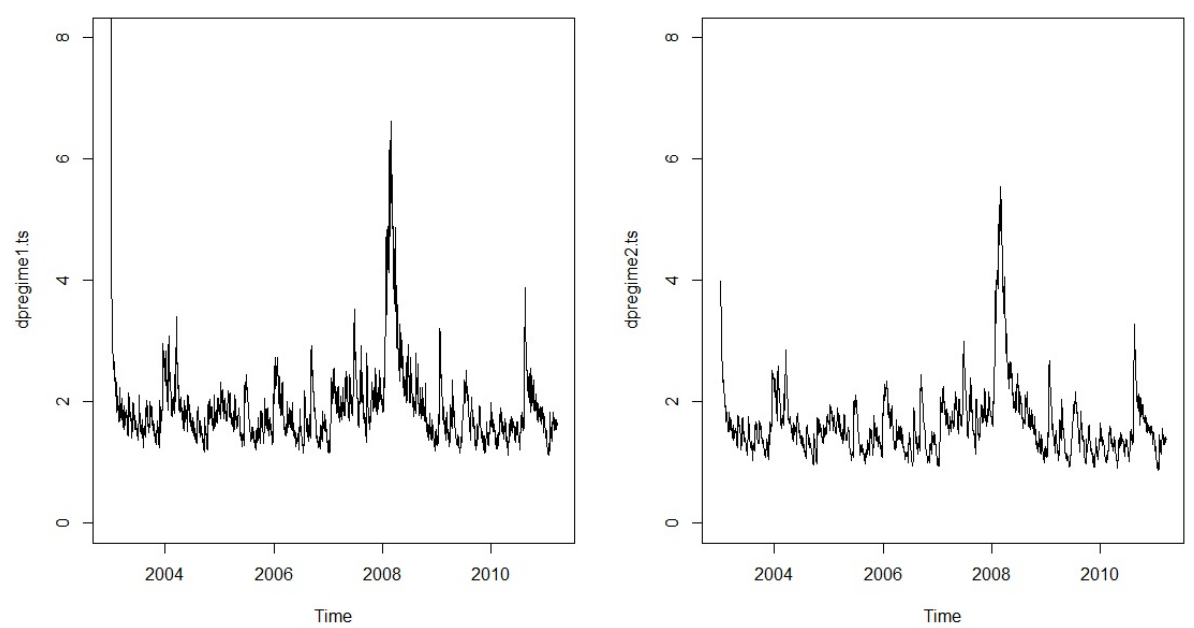

Figura 5.11: Estimativa do desvio padrão condicional para os retornos do Ibovespa no Regime 1 e 2 com erro GED.

\subsection{Análise de Resíduos-IBOVESPA}

O modelo MRS-GARCH com $\epsilon_{t}$ normal, $t$-Student ou GED foi bem ajustado, se os resíduos padronizados

$$
\widetilde{r_{t}}=r_{t} / \sqrt{h_{t}}
$$

comportam-se como variáveis aleatorias i.i.d com distribuição normal padrão, $t$-Student ou GED. Como no caso de modelos GARCH, usualmente supomos que os $\epsilon_{t}$ são normais, ou seguem uma distribuição $t$-Student, ou ainda, uma distribuição de erro generalizada. 


\subsubsection{Análise de Resíduos para o modelo MRS-GARCH-N}
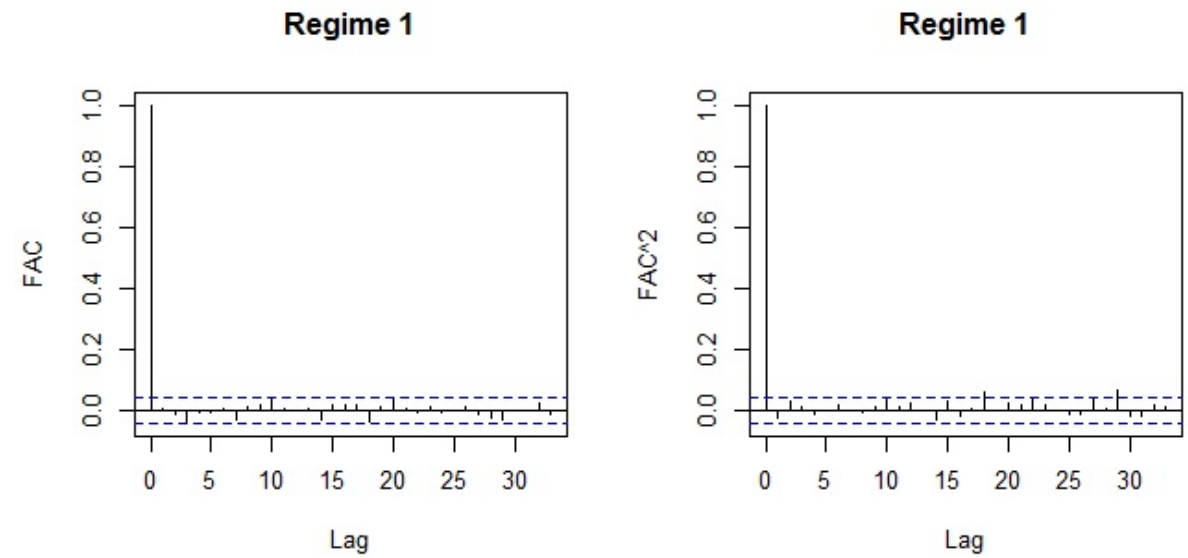

Regime 2

Regime 2
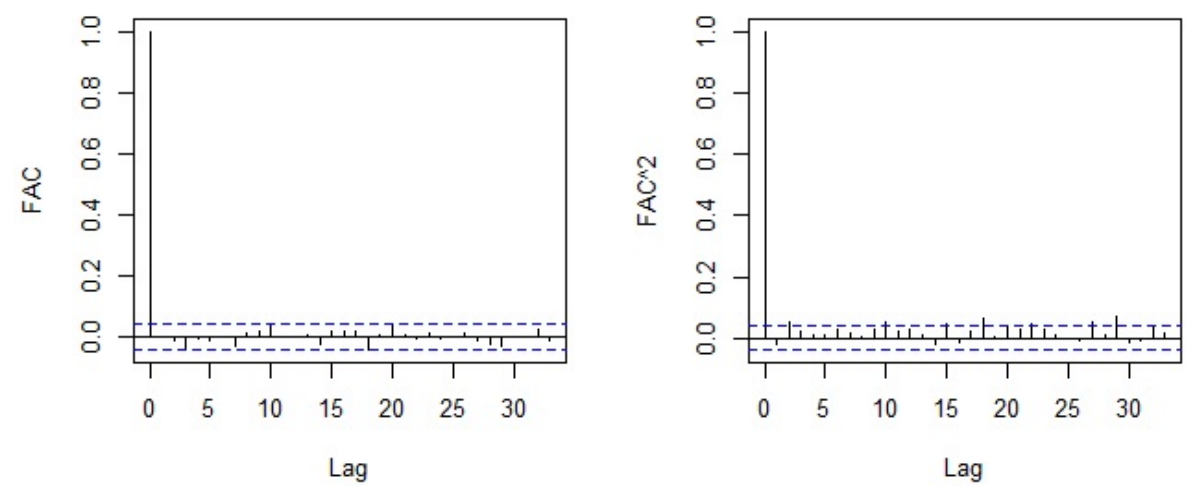

Figura 5.12: Função de autocorrelação e função de autocorrelação ao quadrado dos resíduos padronizados do modelo Normal-Ibovespa.
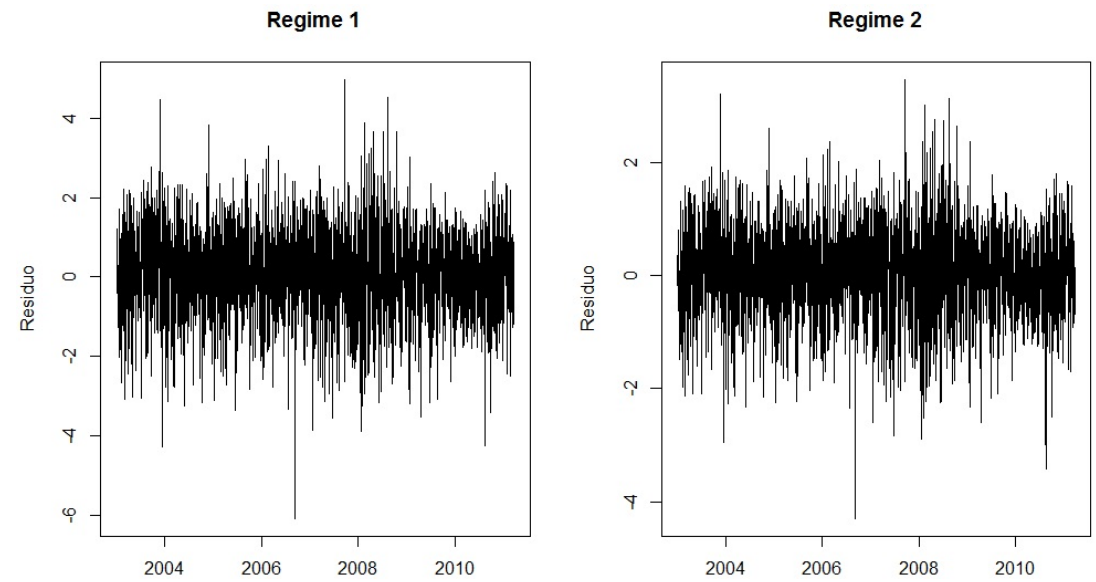

Figura 5.13: Resíduos padronizados nos Regimes 1 e 2 do modelo Normal Ibovespa. 

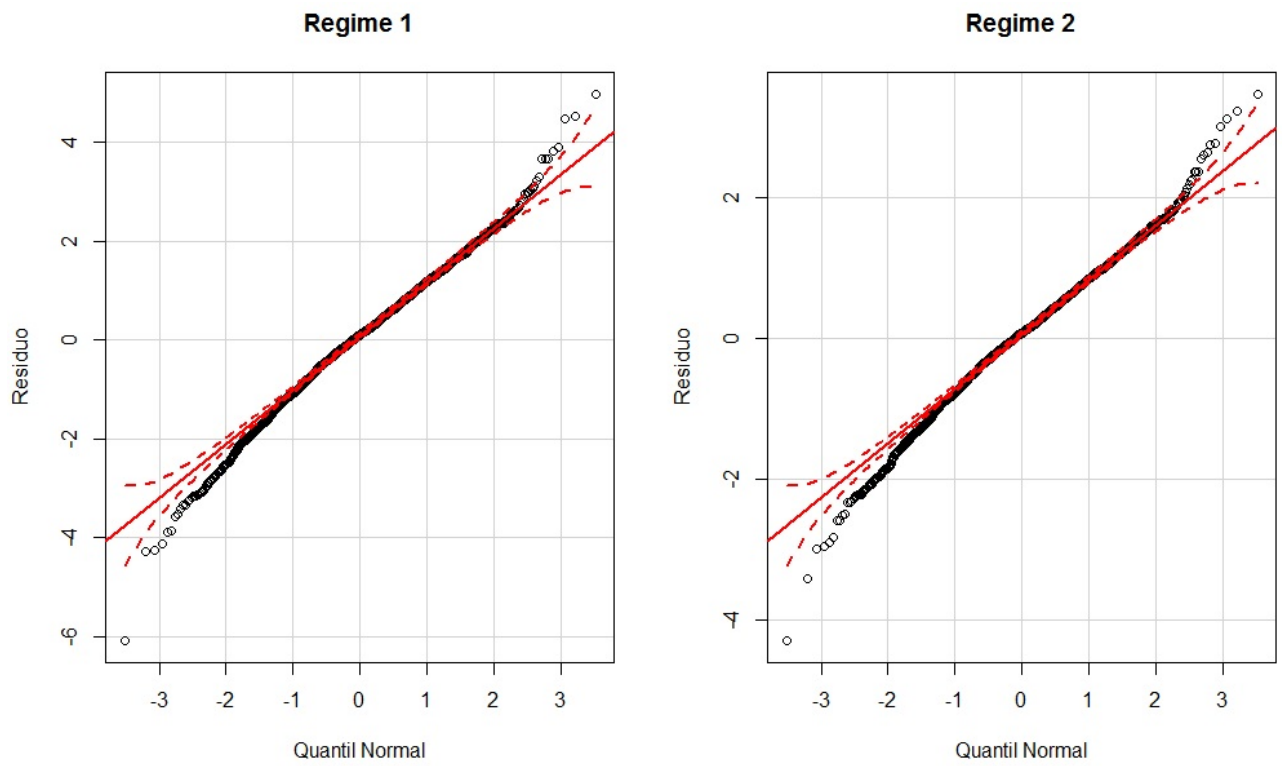

Figura 5.14: Gráfico de quantis dos Residuos padronizados do modelo Normal-Ibovespa.

Histograma dos Residuos Regime 1

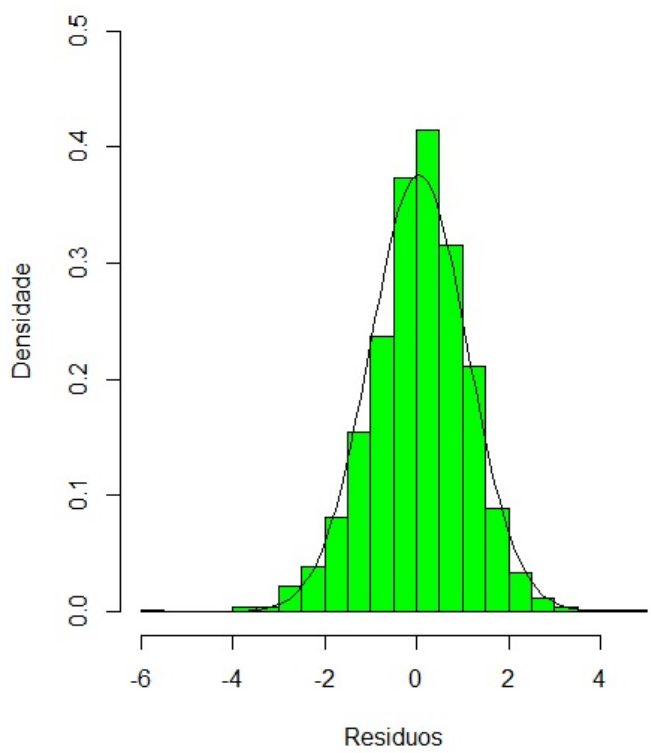

Histograma dos Residuos Regime 2

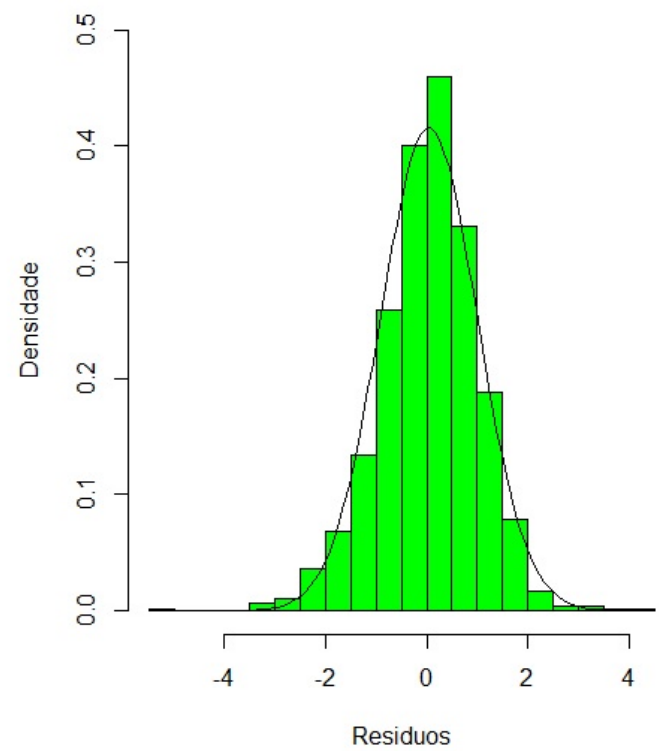

Figura 5.15: Histograma dos resíduos padronizados do modelo Normal-Ibovespa. 


\subsubsection{Análise de Resíduos para o modelo MRS-GARCH-t2}

Regime 1

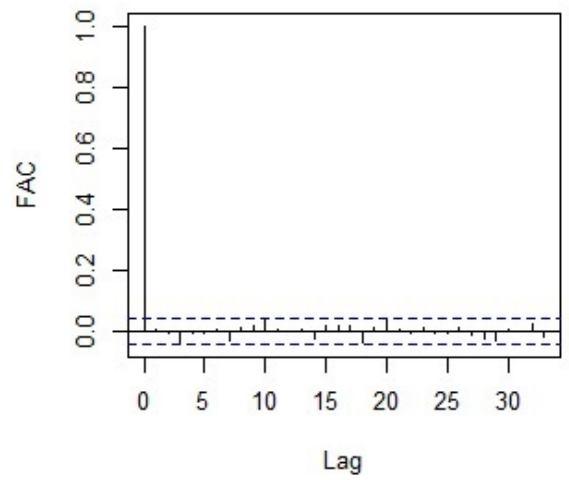

Regime 2

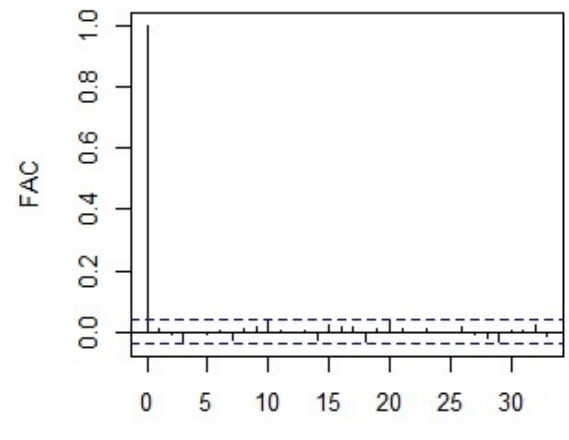

Lag
Regime 1

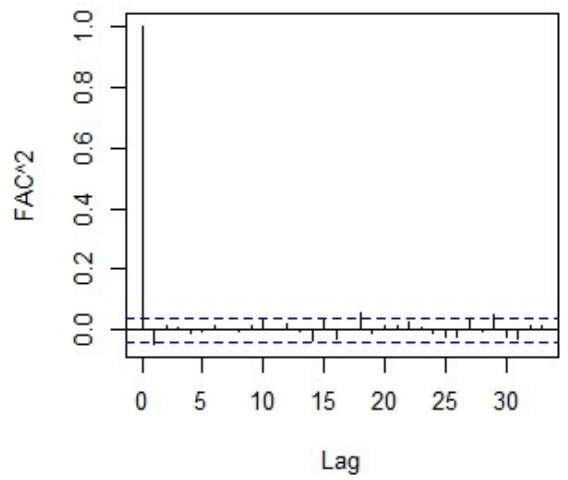

Regime 2

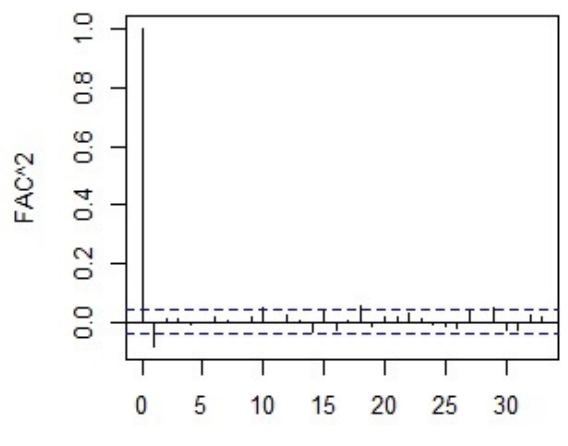

Lag

Figura 5.16: Função de autocorrelação e função de autocorrelação ao quadrado dos resíduos padronizados do modelo t2-Ibovespa.
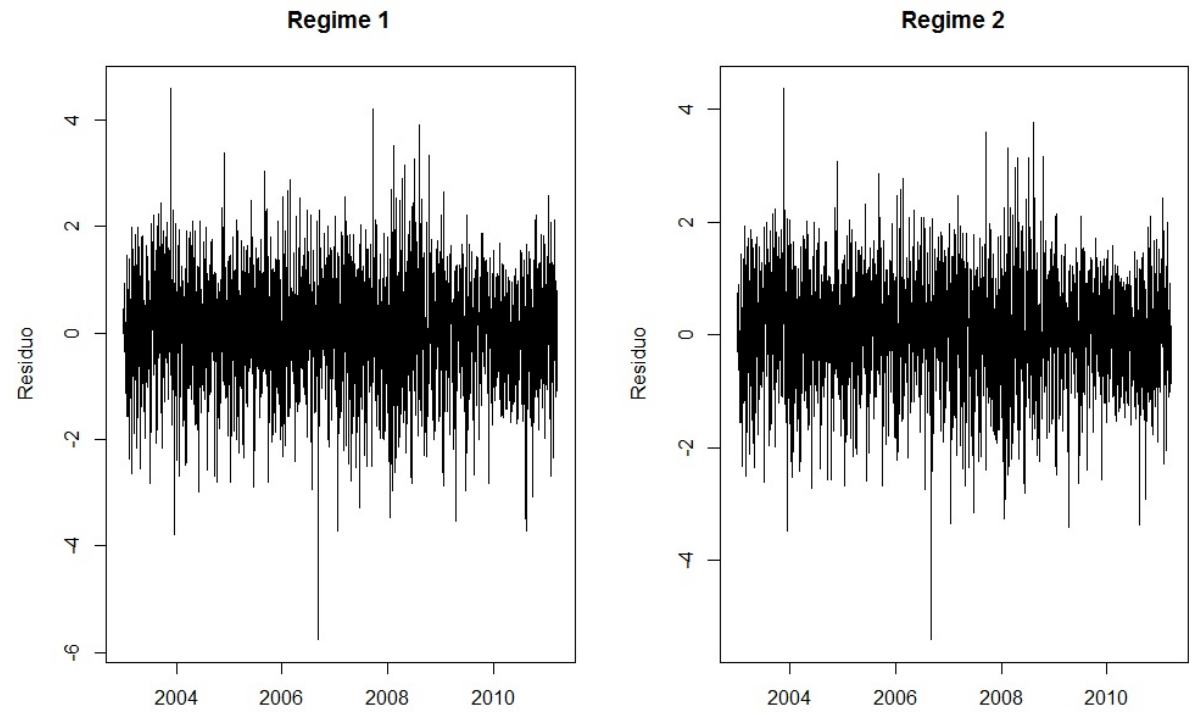

Figura 5.17: Resíduos padronizados nos Regimes 1 e 2 do modelo t2-Ibovespa. 

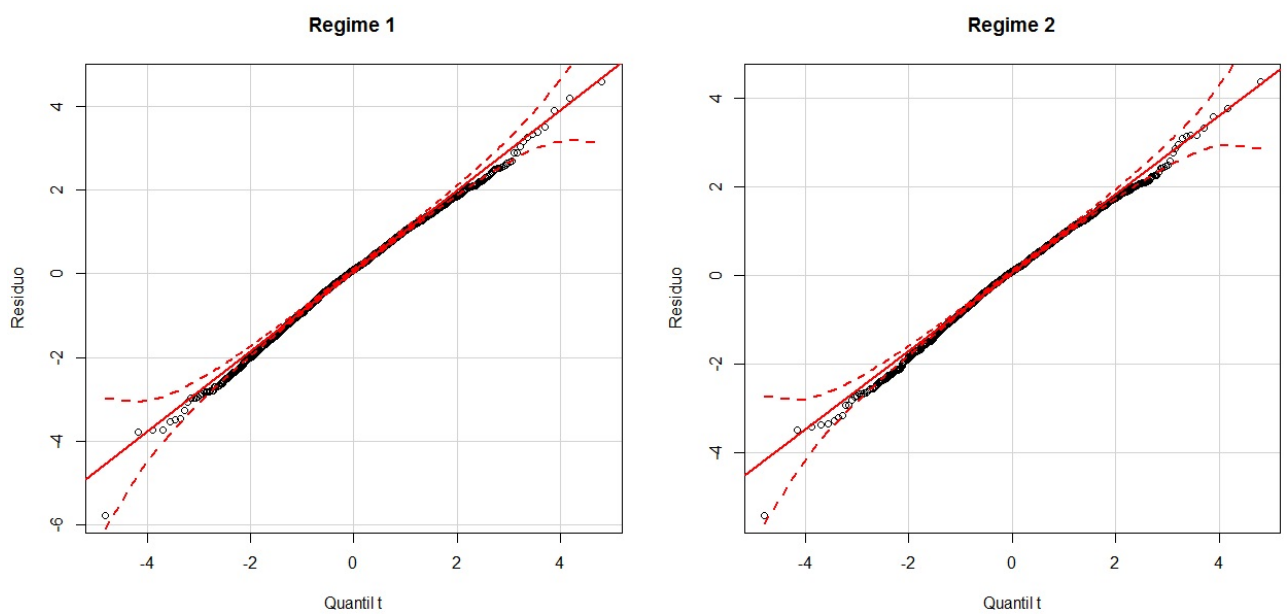

Figura 5.18: Gráfico de quantis dos Resíduos padronizados do modelo t2-Ibovespa.

Histograma dos Residuos Regime 1

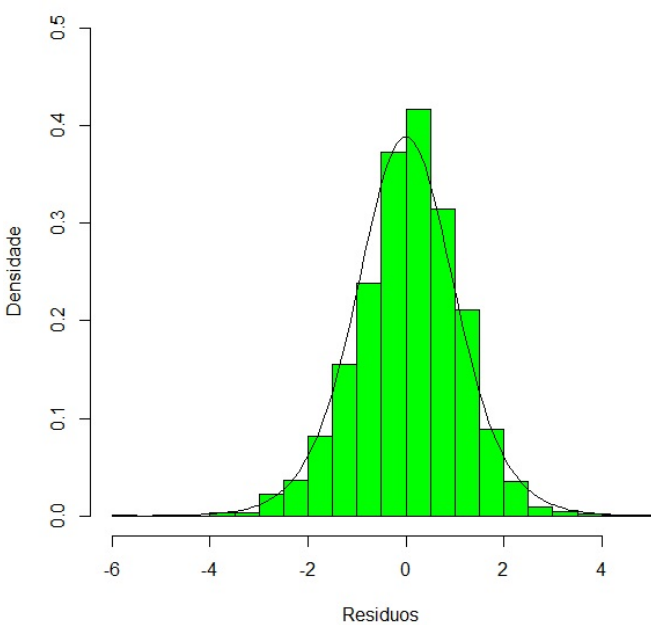

Histograma dos Residuos Regime 2

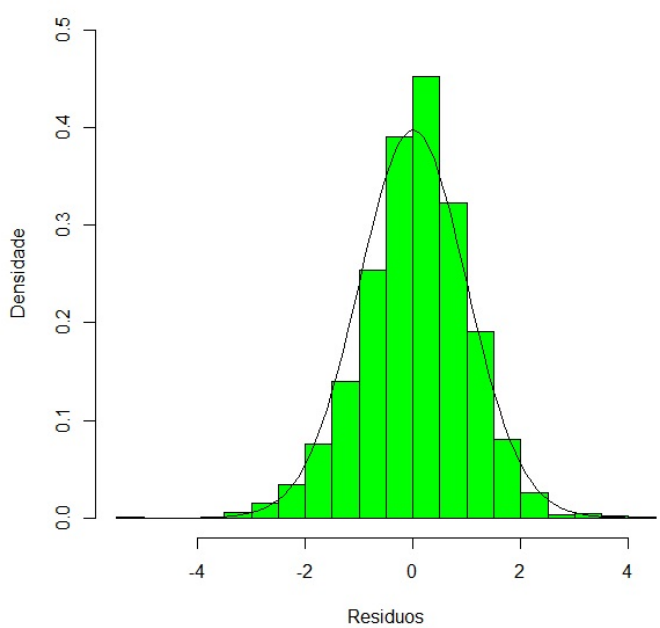

Figura 5.19: Histograma dos Resíduos padronizados do modelo t2-Ibovespa. 


\subsubsection{Análise de Resíduos para o modelo MRS-GARCH-t}

Regime 1

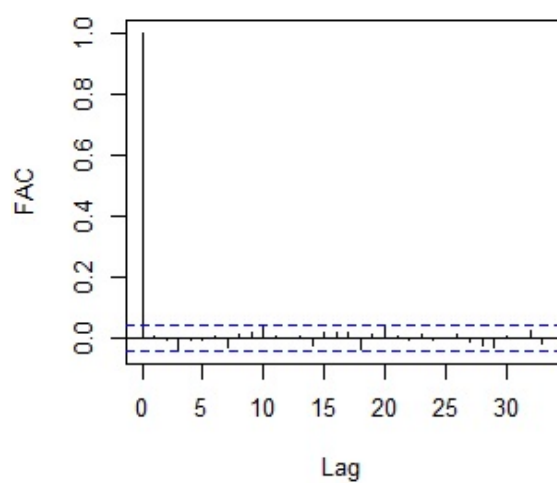

Regime 2

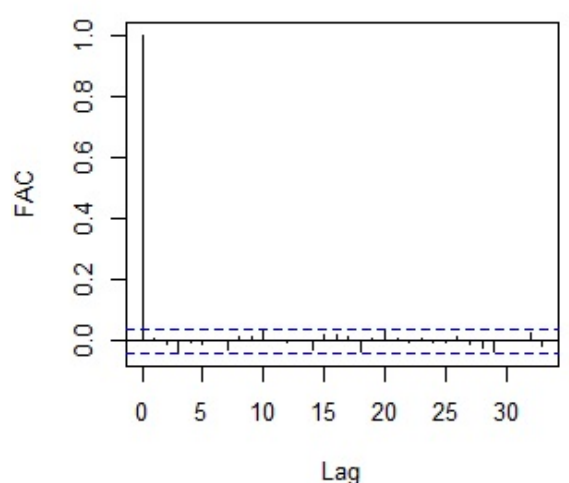

Regime 1

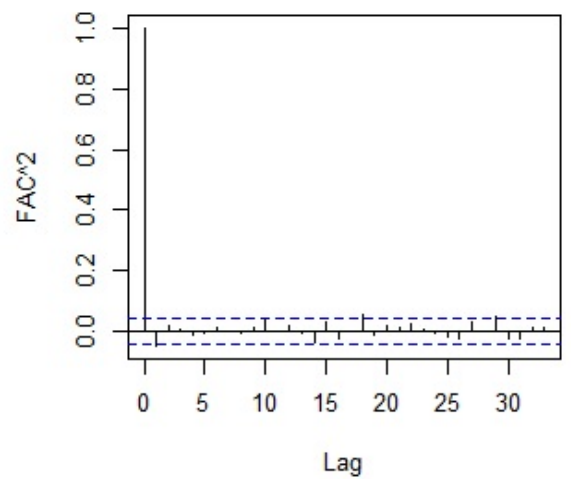

Regime 2

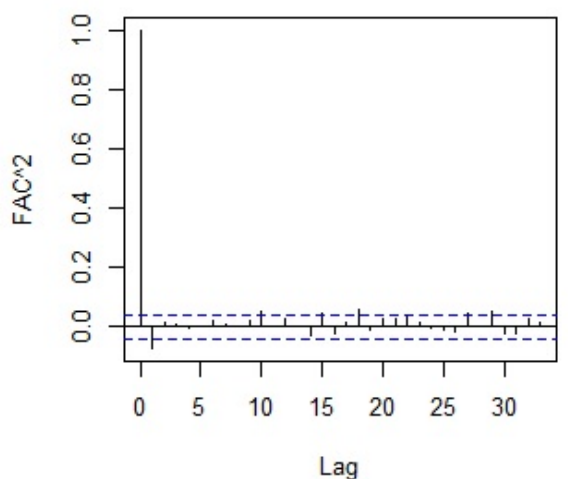

Figura 5.20: Função de autocorrelação e função de autocorrelação ao quadrado dos resíduos padronizados do modelo t-Ibovespa.
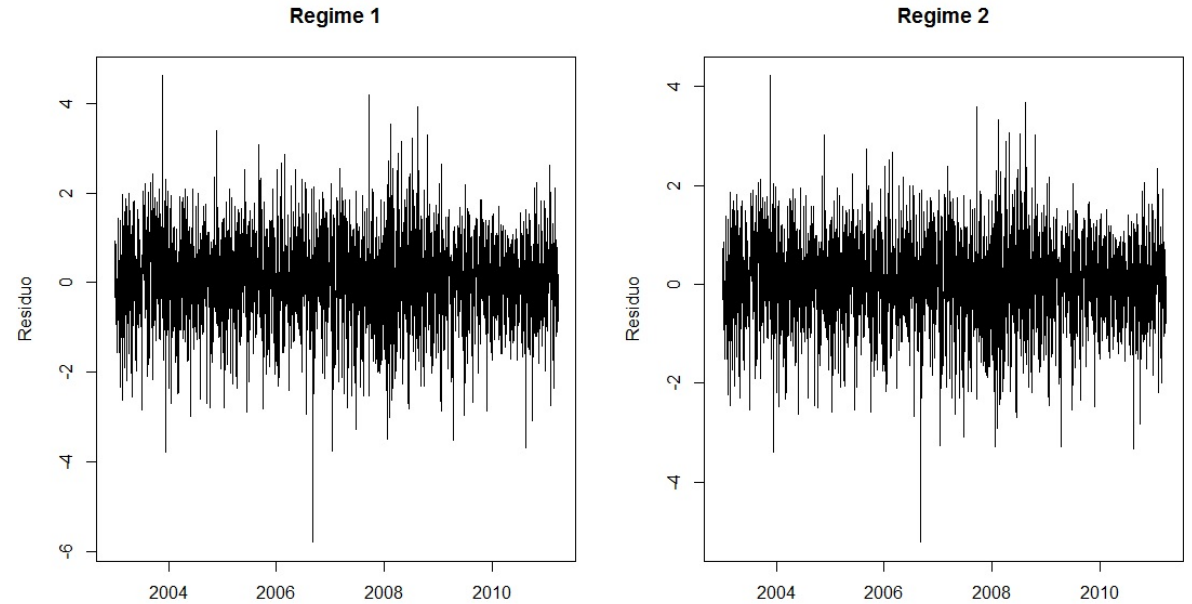

Figura 5.21: Resíduos padronizados nos Regimes 1 e 2 do modelo t-Ibovespa. 

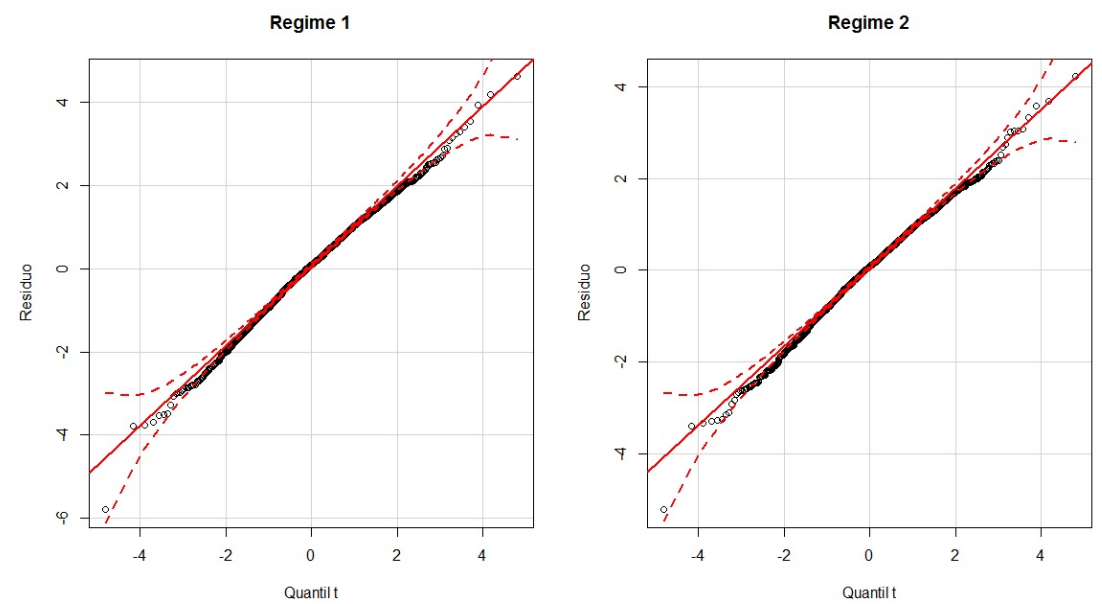

Figura 5.22: Gráfico de quantis dos Resíduos padronizados do modelo t-Ibovespa.

Histograma dos Residuos Regime 1

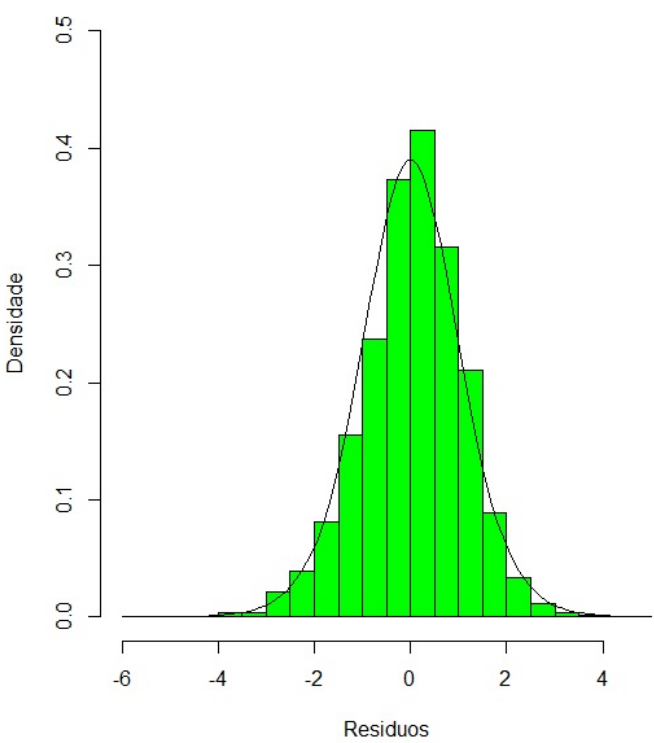

Histograma dos Residuos Regime 2

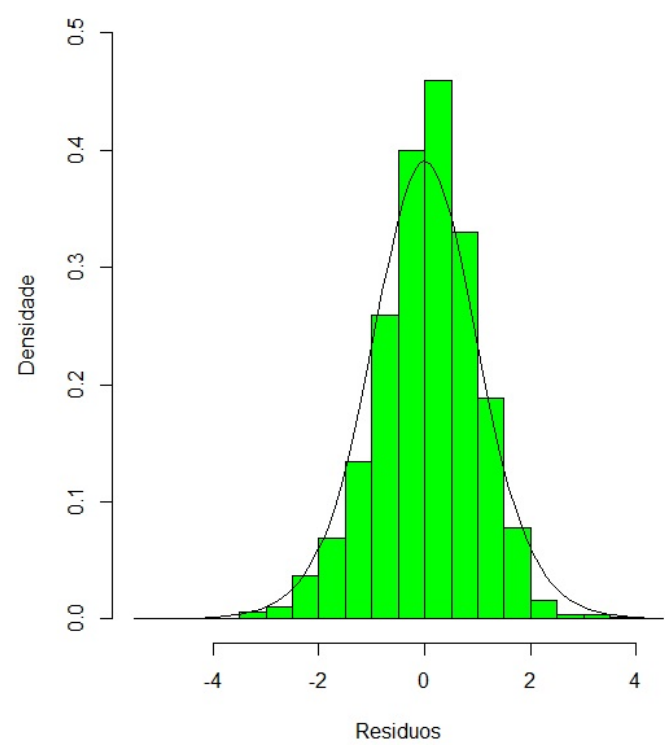

Figura 5.23: Histograma dos Resíduos padronizados do modelo t-Ibovespa. 


\subsubsection{Análise de Resíduos para o modelo MRS-GARCH-GED}

Regime 1

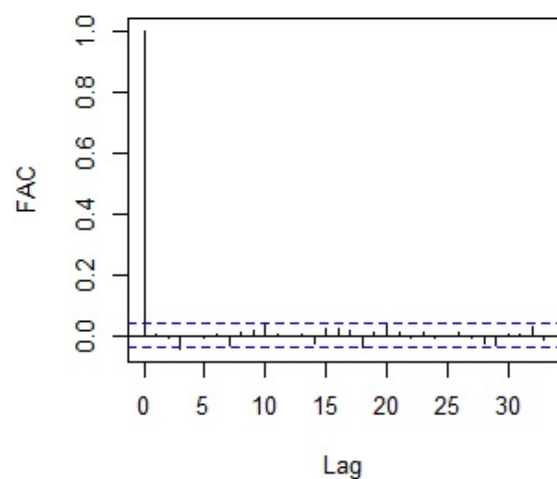

Regime 2

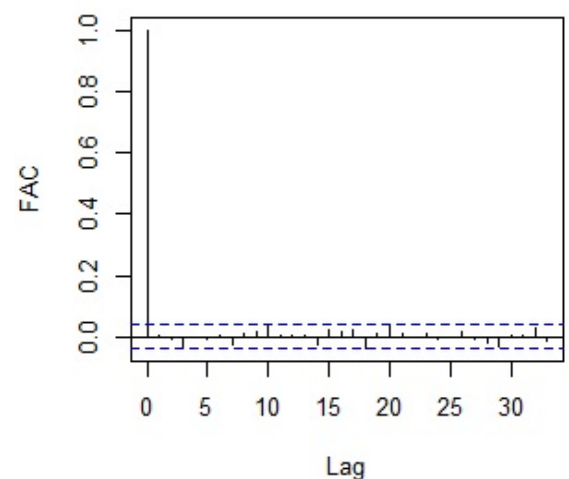

Regime 1

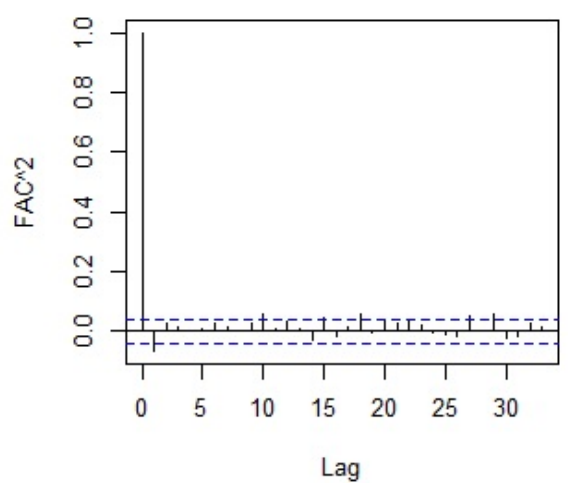

Regime 2

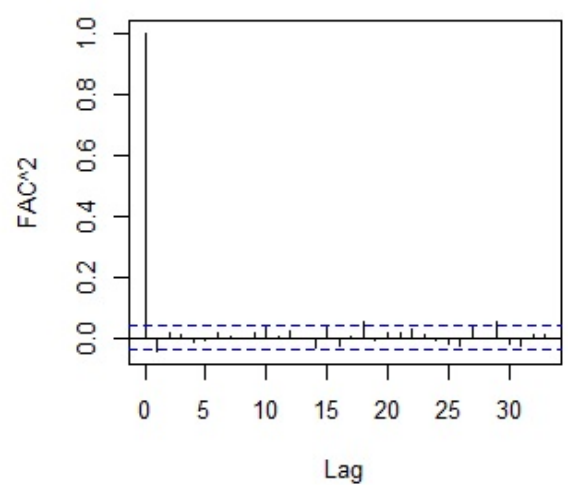

Figura 5.24: Função de autocorrelação e função de autocorrelação ao quadrado dos resíduos padronizados do modelo GED-Ibovespa.
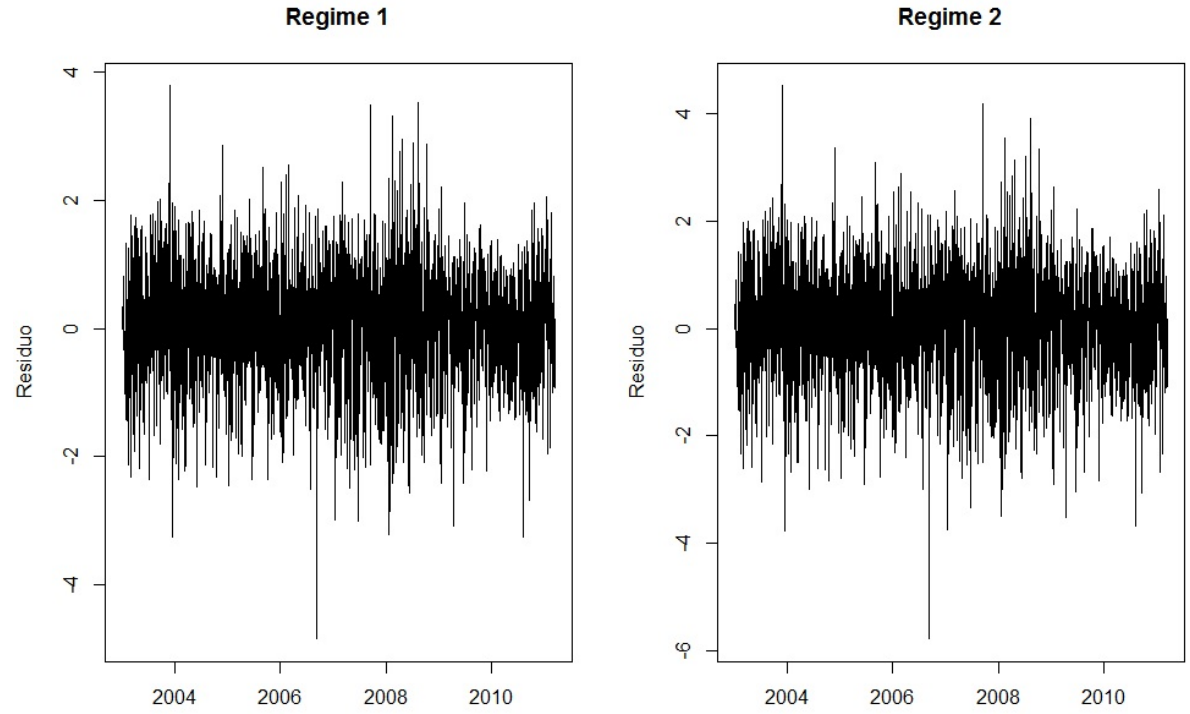

Figura 5.25: Resíduos padronizados nos Regimes 1 e 2 do modelo GED-Ibovespa. 

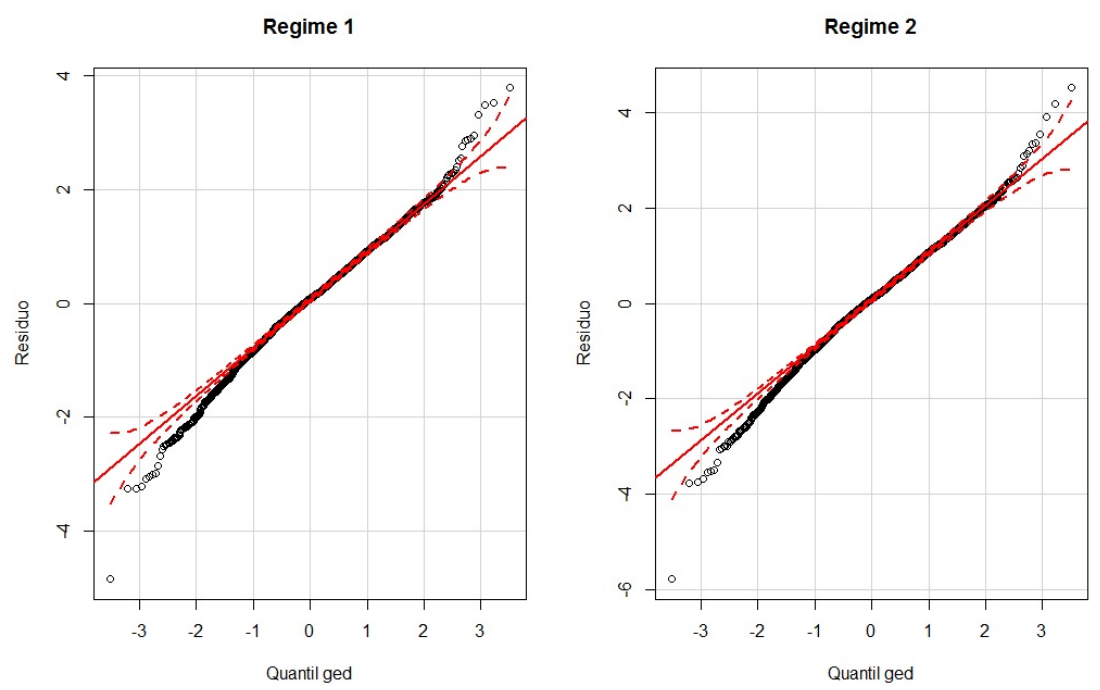

Figura 5.26: Gráfico de quantis dos Resíduos padronizados do modelo GED-Ibovespa.

Histograma dos Residuos Regime 1

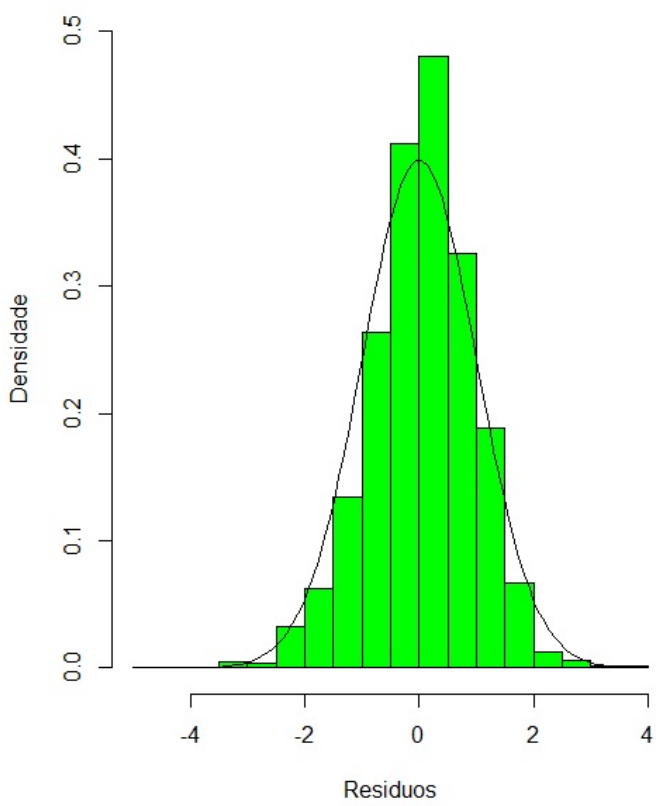

Histograma dos Residuos Regime 2

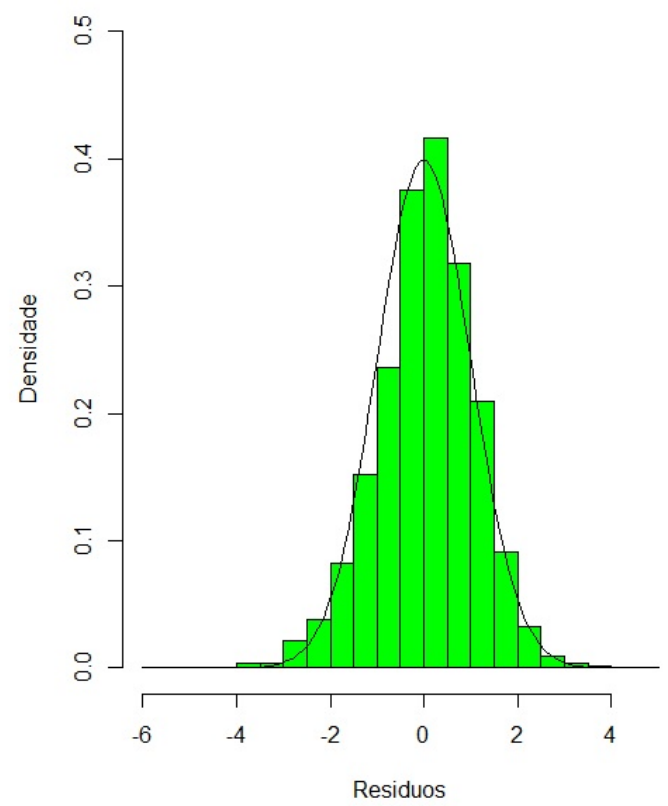

Figura 5.27: Histograma dos Resíduos padronizados do modelo GED-Ibovespa.

A análise de resíduos tem o objetivo de verificar a validade das suposições dos modelos de forma geral. Assim, a escolha do melhor modelo depende dessa análise de resíduos, utilizando os resíduos do regime 1 e 2 . Os modelos supõem que os erros são não correlacionados e seguem a distribuição especificada.

Portanto, verificamos se os resíduos padronizados são não correlacionados, se os resíduos padronizados ao quadrado também são não correlacionados e se seguem a distribuição normal, t de Student ou GED dependendo do modelo. Os gráficos de quantis indicam que somente os modelos com distribuição t de Student com distintos graus de liberdade são adequados e por isso esses modelos foram escolhidos para os log retornos do Ibovespa. A função de autocorrelação parcial dos resíduos padronizados não indicam nenhuma quebra de comportamento de ruído branco nos resíduos. 
Em seguida, avaliaremos o desempenho dos modelos com base na cobertura dos intervalos de confiança para os log retornos.
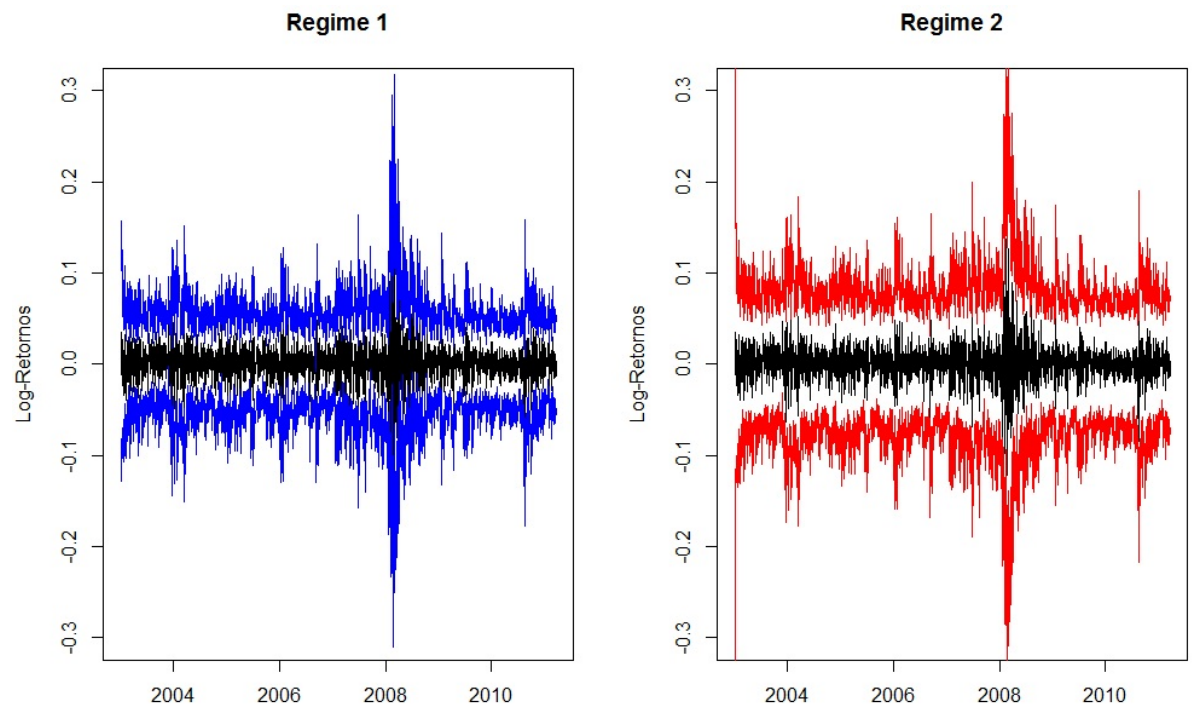

Figura 5.28: Retornos e Intervalos de confiança (95\%)-Normal-Ibovespa.
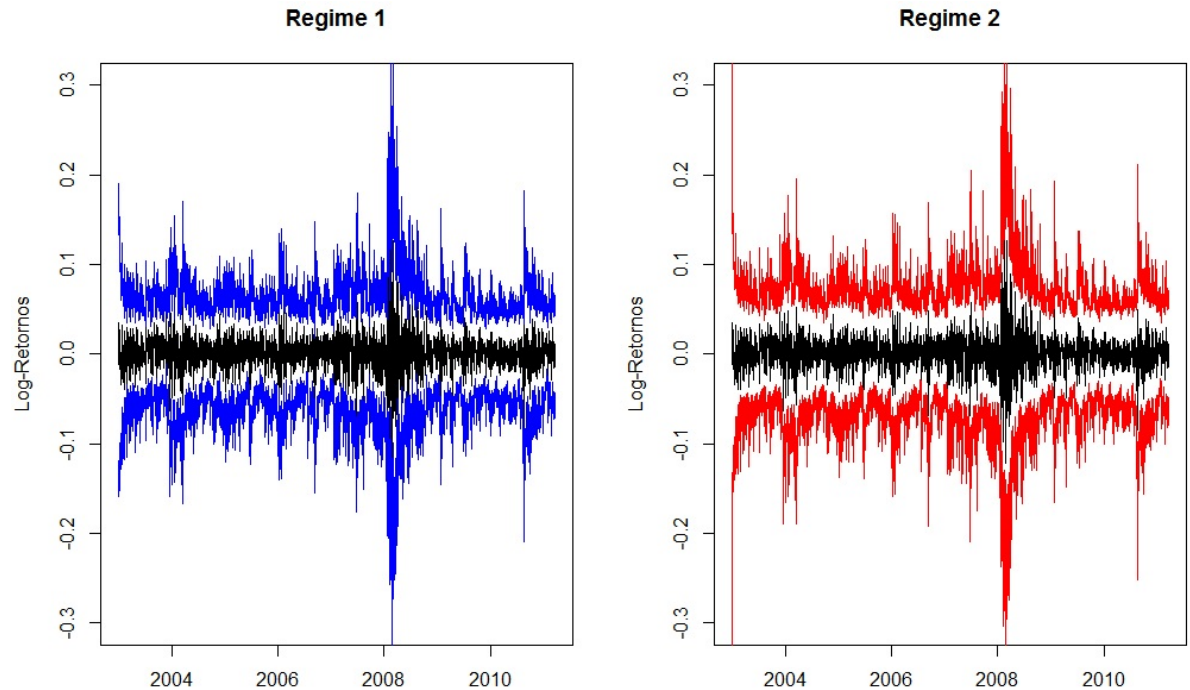

Figura 5.29: Retornos e Intervalos de confiança (95\%)-t-Ibovespa. 

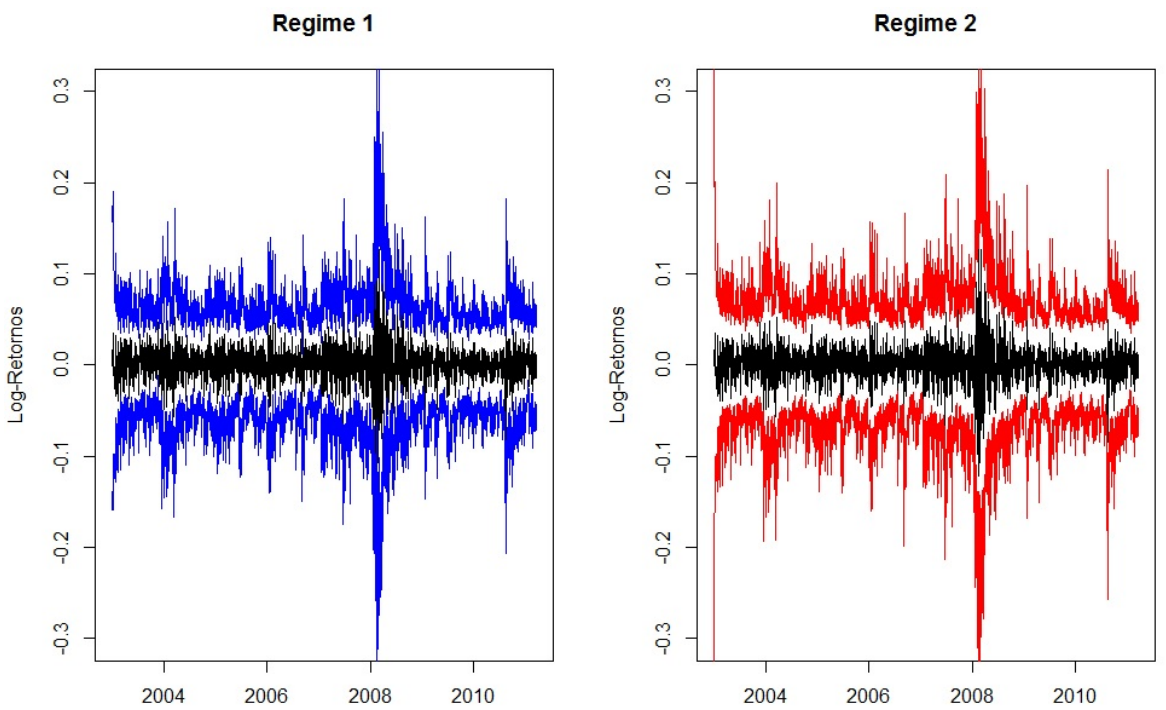

Figura 5.30: Retornos e Intervalos de confiança (95\%)-t2-Ibovespa.
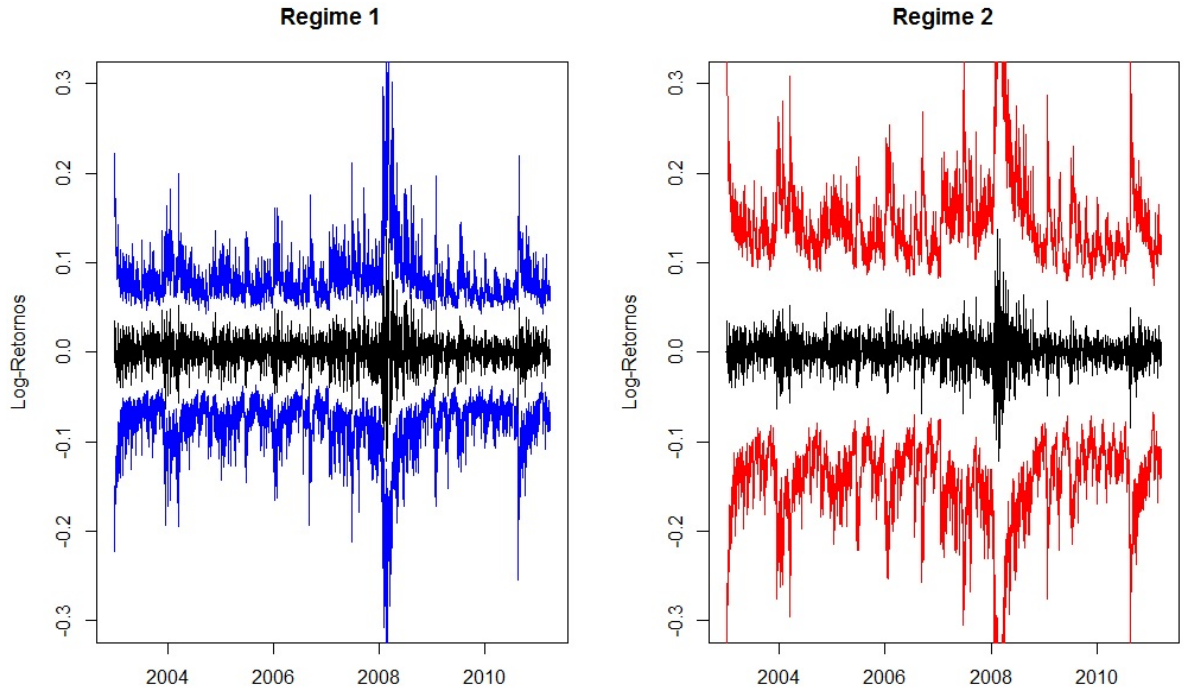

Figura 5.31: Retornos e Intervalos de confiança (95\%)-GED-Ibovespa. 
Tabela 5.3: Porcentagem de Cobertura do intervalos de confiança para os log-retornos do Ibovespa

\begin{tabular}{ccccc}
\hline & Cobertura (\% ) & Comp. Médio & Mínimo & Máximo \\
\hline GARCH(1,1)-N & 95 & 6.79 & 4.24 & 25.46 \\
2R.GARCH(1,1)-N & 98 & 6.23 & 3.81 & 25.32 \\
\hline GARCH(1,1)-t & 98 & 8.69 & 5.40 & 32.40 \\
2R.GARCH(1,1)-t & 99 & 7.24 & 3.74 & 24.45 \\
\hline GARCH(1,1)-t & 98 & 8.69 & 5.40 & 32.40 \\
2R.GARCH(1,1)-t2 & 99 & 7.25 & 3.80 & 24.70 \\
\hline GARCH(1,1)-GED & 97 & 7.80 & 5.10 & 28.81 \\
2R.GARCH(1,1)-GED & 98 & 7.64 & 4.55 & 35.70 \\
\hline
\end{tabular}

Na Tabela 5.3 mostra-se o modelo Garch usual como $\operatorname{GARCH}(1,1)$ para cada distribuição, e 2R.GARCH $(1,1)$ para o modelo Garch com dois regimes.

Os intervalos de confiança foram obtidos da mesma forma que são calculados para a média de uma população com variância conhecida com o respetivo quantil para cada distribuição especificada, utilizando as estimativas da média e da variância em cada regime. Aqui os intervalos de confiança foram calculados para os log-retornos do Ibovespa.

A cobertura foi calculada utilizando o valor do log-retorno maior do que o limite inferior de confiança para observar se ele se encontra dento do intervalo ou não. A porcentagem de cobertura é simplesmente calculada como a média desses valores. Os intervalos apresentaram cobertura maior que $95 \%$ e as maiores coberturas foram para as distribuições t de Student com distintos graus de liberdade. O comprimento foi calculado fazendo a diferença entre o limite superior de confiança com o limite inferior de confiança e o comprimento médio é simplesmente a média do comprimento. Aquí o Mínimo é o valor mínimo no comprimento e o máximo é o valor máximo do comprimento. Pode-se observar que os valores para os modelos com 2 regimes tem menor comprimento médio do que os modelos com 1 regime. 


\section{$5.4 \quad$ S\&P500}

O conjunto de dados analisado neste trabalho é o índice S\&P500. O período considerado é de 2 de janeiro de 2003 a 9 de abril de 2012 para um total de 2333 observações. Os dados foram obtidos a partir do site yahoo finanças. A cotação estudada foi o preço de fechamento diário do índice.

Primeiramente, apresentamos nas Figuras 5.32 e 5.33, a série S\&P500, a série de retornos, o gráfico de quantis e histograma dos retornos.
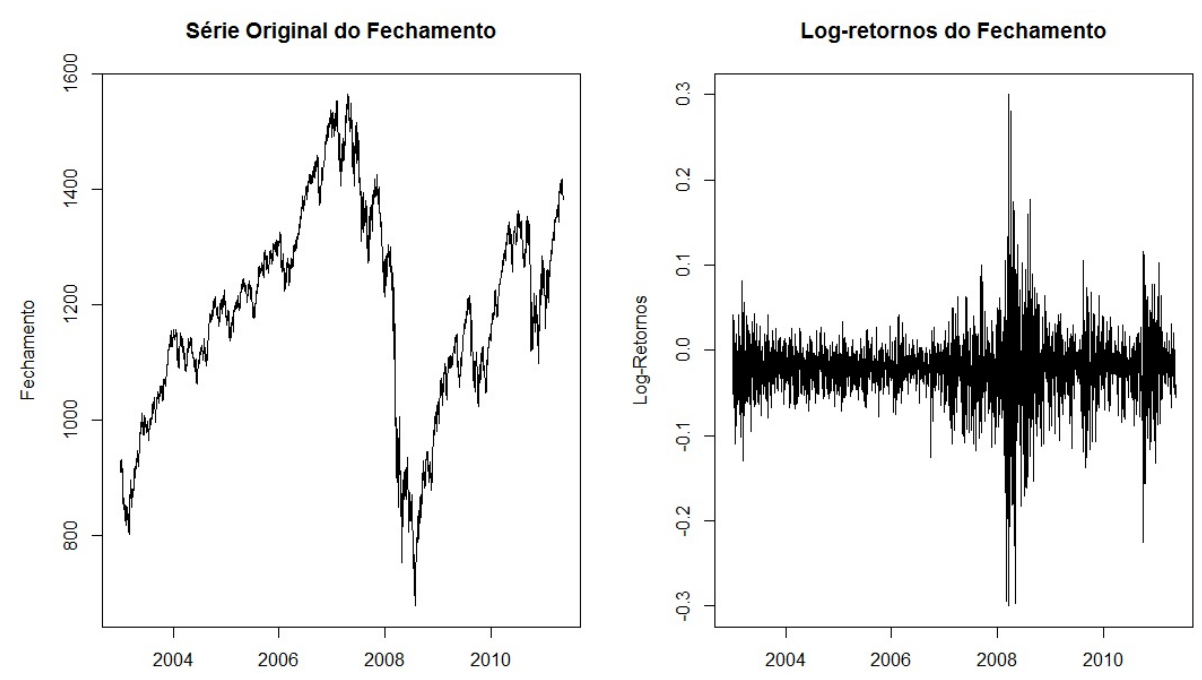

Figura 5.32: Gráfico da Série S\&P500 e os Log-retornos.
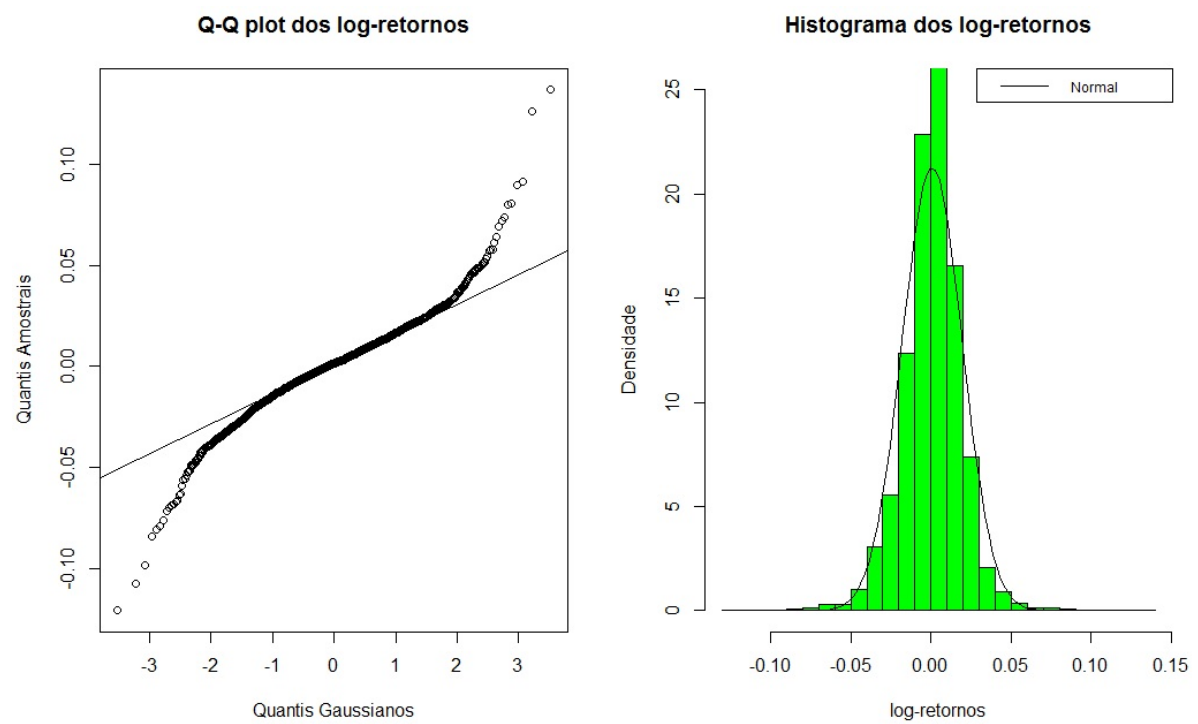

Figura 5.33: Gráfico de quantis e Histograma dos log-retornos da Série S\&P500.

A Tabela 5.4 mostra algumas estatísticas descritivas dos log-retornos do Índice S\&P500. Note que a média dos dados está próxima de zero e o desvio padrão é pequeno, mas os log-retornos variam de -0.09 a 0.10 . A média é próxima de zero e, com o desvio padrão pequeno, podemos concluir que os dados se encontram aglomerados em torno do zero, o que pode ser confirmado pelo histograma dos retornos diários do S\&P500. Observando o gráfico da série diária do S\&P500, podemos ver a que a série é não estacionária, pois apresenta tendência não constante e variabilidade não controlada. Já o gráfico dos retornos diários do S\&P500, apresenta nível, aparentemente, constante e variabilidade controlada. A assimetria e a curtose indicam que os dados não seguem uma distribuição normal. Com base nessas medidas resumo dos retornos diários da S\&P500, podemos observar que: a distribuição dos retornos possui uma leve assimetria à esquerda, já que o valor do coeficiente de assimetria é 
Tabela 5.4: Medidas descritivas do retorno-SEP500

\begin{tabular}{lllllll}
\hline Média & Mediana & D.Padrão & Curtose & Mínimo & Máximo & Assimetria \\
\hline 0.00017 & 0.00081 & 0.01341 & 9.95099 & -0.09451 & 0.10950 & -0.29579 \\
\hline
\end{tabular}

não nulo e negativo e a medida de curtose da distribuição dos retornos indica que sua curva é mais achatada que a curva da distribuição Normal, ou seja, há uma maior quantidade de dados nas caudas da curva da distribuição dos retornos do que em uma curva da distribuição Normal. A assimetria e a curtose podem ser visualizadas no gráfico de quantis e histograma da Figura 5.33.

Series Lnret

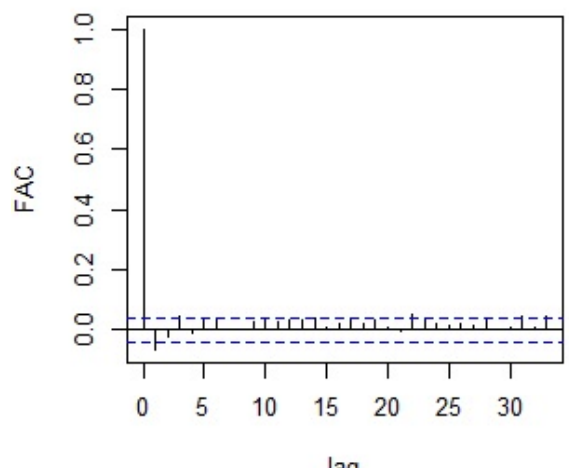

Series Lnret2

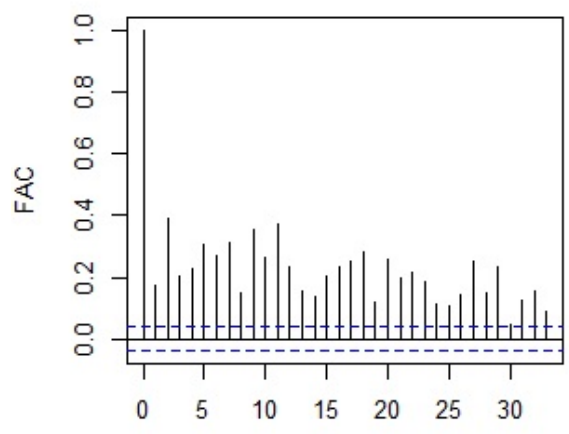

lag
Series Lnret

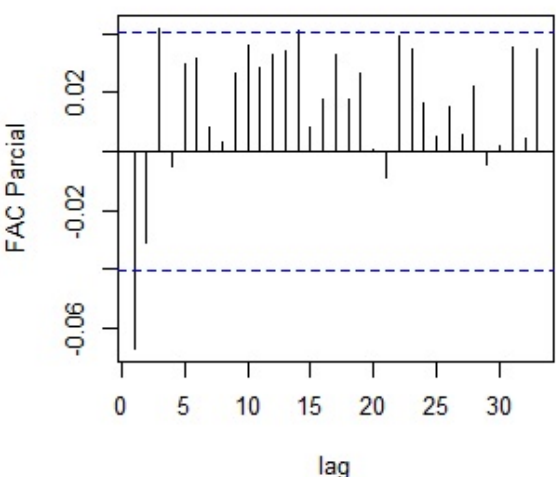

Series Lnret2

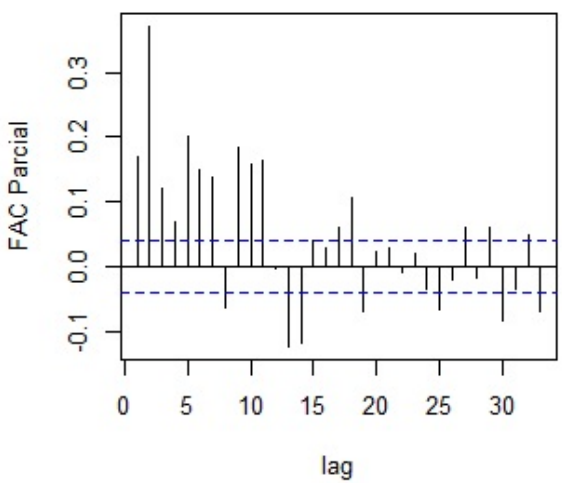

Figura 5.34: Função de autocorrelação dos log-retornos e log-retornos ao quadrado-SËP500.

Entretanto, na Figura 5.34 observa-se que as autocorrelações dos retornos são não significativas e as autocorrelações dos retornos ao quadrado são significativas. Conforme descrito no Capítulo 3, este comportamento indica que a série de retornos pode mostrar presença de heterocedasticidade condicional nos dados, una vez que os retornos ao quadrado medem o momento de segunda ordem da série original, este resultado indica que a variância dos retornos da série da S\&P500 condicionada ao passado histórico pode sofrer alterações ao longo do tempo.

As estimativas dos parâmetros do modelo MRS-GARCH são apresentadas na Tabela 5.5. Foram ajustados os modelos com distribuição Normal, t de Student e a distribuição generalizada do erro e para o modelo com distribuição t de Student foi considerado o modelo em que o grau de liberdade pode ser diferente nos 2 regimes (t2). Em particular, a Tabela 5.5 mostra o desvio padrão condicional dos retornos para cada regime da volatilidade, isto é 


$$
\sigma^{(i)}=\left(a_{0}^{(i)} /\left(1-a_{1}^{(i)}-b_{1}^{(i)}\right)\right)^{1 / 2} .
$$

Para o modelo t de Student (t2), o parâmetro da média é significativo para o regime 1 e não significativo para o regime 2, no caso da distribuição normal e GED os parâmetros da média são negativos para o regime 1 . O parâmetro $a_{1}$, que mede o efeito do erro anterior na variância, parece ser significativo para os três modelos considerados.

Para o regime 1, as estimativas de $a_{1}$ são maiores do que no regime 2 que são quase zero para o modelo normal e GED. Entretanto, os valores para os parâmetros $b_{1}$ são maiores para o regime 1 no modelo normal e GED, já para o modelo t de Student esse valor é menor o que significa que temos mais peso para cada erro de ontem e menor inercia i.e para a variância anterior $\sigma_{t-1}^{2}$. A probabilidade de se manter no regime $1, p_{11}$ é menor (49\% para a distribuição t2), que a probabilidade de se manter no regime 2, o mesmo acontece para a distribuição normal e GED.

A Tabela 5.5 também mostra as probabilidades incondicionais de cada modelo MRS-GARCH. A probabilidade incondicional $\pi_{1}$ de estar no primeiro regime varia entre $27 \%$ para o modelo normal e $47 \%$ para o modelo t de Student com 2 graus de liberdade. Para o modelo $t$-Student com 2 graus de liberdade no primeiro regime, o parâmetro $\nu$ indica que a distribuição tem caudas mais pesadas do que a normal, já para o segundo regime, o valor do parâmetro $\nu$ indica que a distribuição é aproximadamente normal. No caso do GED o valor do parâmetro $\nu$ está indicando que a distribuição tem caudas mais pesadas do que o normal.

A identificação de regime com caudas mais pesadas no regime 2 da distribuição t de Student nos leva a escolher o modelo t2.

Nesta aplicação pode-se percerber que não encontram-se as estimativas para o modelo t de Student, pois no processo de otimização os valores estavam convergindo para números complexos e não estava conseguindo achar o mínimo local, logo não foi considerada esta distribuição para esse estudo. 
Tabela 5.5: Estimativas de Máxima Verossimilhança e erros padrões do Modelo MRS - GARCH - SEP 500

\begin{tabular}{|c|c|c|c|}
\hline & MRS-GARCH-N & MRS-GARCH-t2 & MRS-GARCH-GED \\
\hline \multirow[t]{2}{*}{$\mu^{(1)}$} & -0.3116 & 0.1651 & -0.2380 \\
\hline & $(0.0828)$ & $(0.0248)$ & $(0.0976)$ \\
\hline \multirow[t]{2}{*}{$\mu^{(2)}$} & 0.1021 & 0.0070 & 0.1111 \\
\hline & $(0.0204)$ & $(0.0289)$ & $(0.0210)$ \\
\hline \multirow[t]{2}{*}{$\sigma^{(1)}$} & 0.1338 & 0.0256 & 0.1370 \\
\hline & $(0.0334)$ & $(0.1195)$ & $(0.0469)$ \\
\hline \multirow[t]{2}{*}{$\sigma^{(2)}$} & 0.0247 & 0.0199 & 0.0224 \\
\hline & $(0.0056)$ & $(0.0567)$ & $(0.0071)$ \\
\hline \multirow[t]{2}{*}{$a_{1}^{(1)}$} & 0.0738 & 0.2504 & 0.0761 \\
\hline & $(0.0240)$ & $(0.0513)$ & $(0.0337)$ \\
\hline \multirow{2}{*}{$a_{1}^{(2)}$} & $<0.0001$ & 0.0248 & $<0.0001$ \\
\hline & $(0.0185)$ & $(0.0281)$ & $(0.0218)$ \\
\hline \multirow{2}{*}{$b_{1}^{(1)}$} & 0.9238 & 0.7108 & 0.9213 \\
\hline & $(0.0303)$ & $(0.1419)$ & $(0.0421)$ \\
\hline \multirow[t]{2}{*}{$b_{1}^{(2)}$} & 0.8980 & 0.9631 & 0.9018 \\
\hline & $(0.0178)$ & $(0.0808)$ & $(0.0206)$ \\
\hline \multirow[t]{2}{*}{$p_{11}$} & 0.9046 & 0.4932 & 0.9088 \\
\hline & $(0.0218)$ & $(0.1831)$ & $(0.0267)$ \\
\hline \multirow[t]{2}{*}{$p_{22}$} & 0.9631 & 0.5391 & 0.9620 \\
\hline & $(0.0084)$ & $(0.1005)$ & $(0.0107)$ \\
\hline \multirow[t]{2}{*}{$\nu^{(1)}$} & - & 2.2599 & 1.5120 \\
\hline & & $(0.0893)$ & $(0.0739)$ \\
\hline \multirow[t]{2}{*}{$\nu^{(2)}$} & $\longrightarrow$ & 172.5236 & $\longrightarrow$ \\
\hline & & $<0.0001$ & \\
\hline $1000 * \log (L)$ & -3.2836 & -3.2561 & -3.2663 \\
\hline$\pi_{1}$ & 0.2789 & 0.4762 & 0.2941 \\
\hline$\pi_{2}$ & 0.7211 & 0.5238 & 0.7059 \\
\hline$\rho_{1}$ & 0.9976 & 0.9612 & 0.9280 \\
\hline$\rho_{2}$ & 0.8980 & 0.9879 & 0.9927 \\
\hline
\end{tabular}


As probabilidades suavizadas do regime 1 com erro gaussiano e os log-retornos podem ser observados no seguinte gráfico:

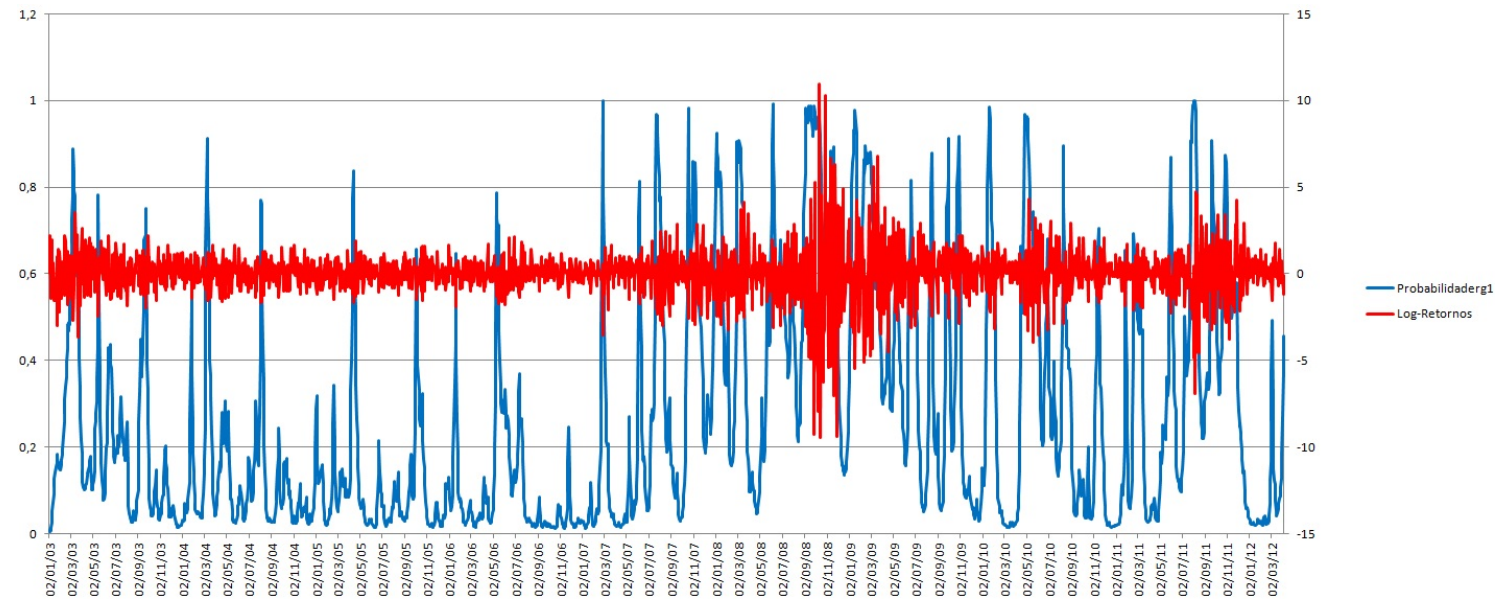

Figura 5.35: Probabilidade suavizada do Regime 1 - Normal - S\&P500.

Em nossa aplicação existem dois regimes, $s_{t}=1$ e $s_{t}=2$, ou seja, se o processo estiver no regime 1, a observação será retirada de uma distribuição normal com média -0.3116 e desvio padrão 0.1338 e se estiver no segundo regime com média 0.1021 e desvio padrão 0.0247. A Figura 5.35 apresenta a probabilidade suavizada do regime 1 e os log-retonos. As probabilidades de estarmos no regime 1 , variam entre 0 e 0.9 , parece haver períodos com menor volatilidade como o ínicio da série até 2007 . As probabilidades de estarmos em um regime e continuarmos neste são $p_{11}$ e $p_{22}$ iguais a 0.9046 e 0.9631 respetivamente. Com isto temos que a matriz de transição e dada por

$$
P=\left(\begin{array}{ll}
0.9046 & 0.0369 \\
0.0954 & 0.9631
\end{array}\right)
$$

Temos então que a probabilidade incondicional de estar no $k$-ésimo regime, neste caso para nosso modelo com 2 regimes é dada por $\pi_{1}=0.2789$ e $\pi_{2}=0.7211$. Ou seja, ao sortearmos uma observação qualquer da série, a probabilidade é maior de estarmos no regime 2 do que no regime 1.

A duração média de estarmos no regime 1 é 10 dias e do regime 2 é 27 dias.
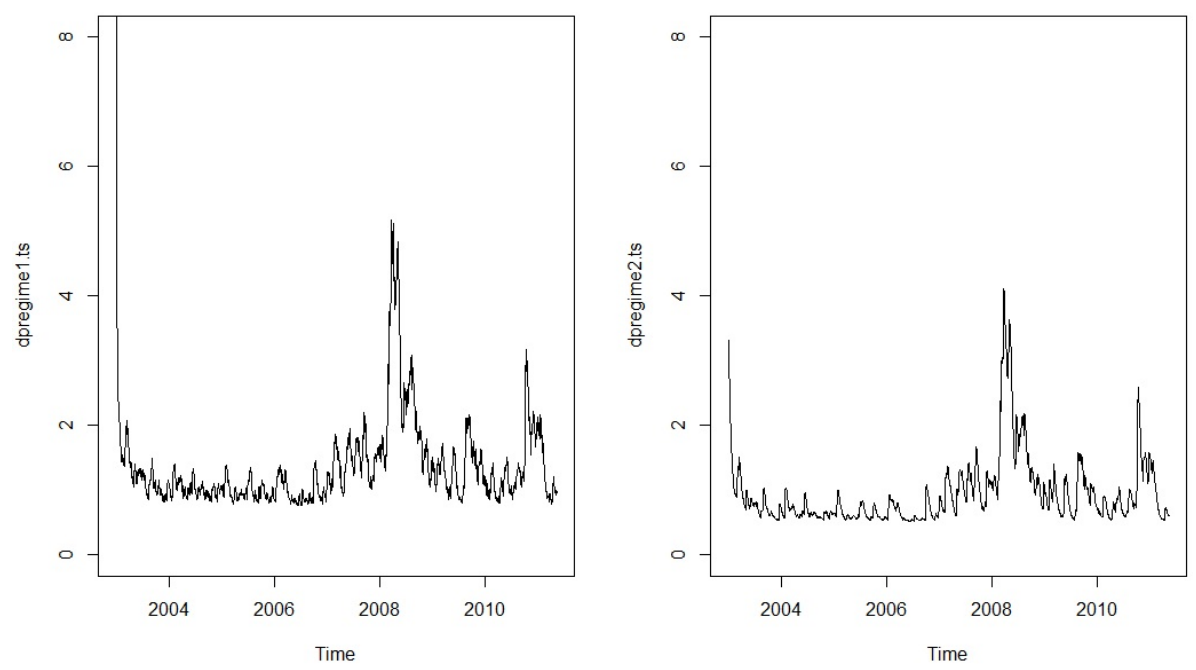

Figura 5.36: Estimativa do desvio padrão condicional para os retornos da S\&SP500 no Regime 1 e 2 com erro gaussiano. 
As probabilidades suavizadas do regime 1 com erro t de Student distintos graus de liberdade e os log-retornos podem ser observados no seguinte gráfico:

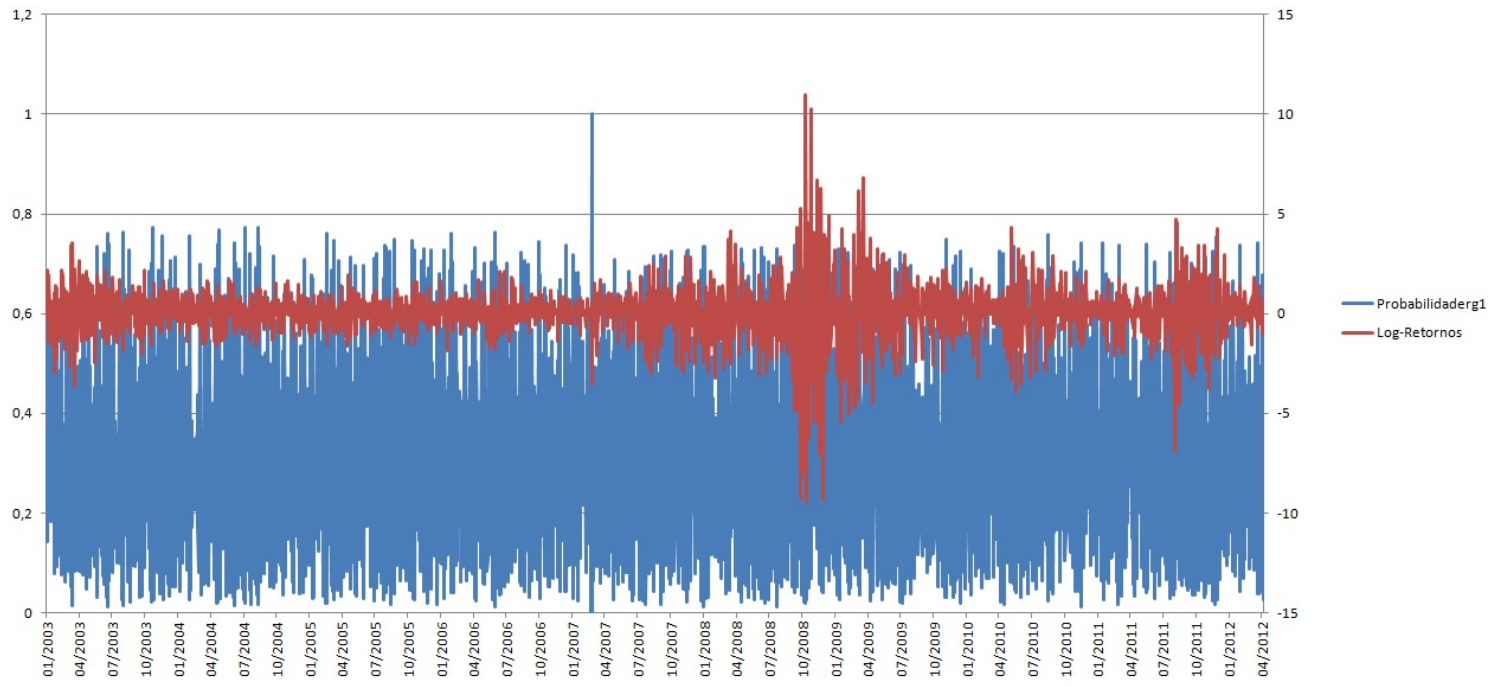

Figura 5.37: Probabilidade suavizada do Regime 1- t2 - S\&P500.

Se o processo estiver no regime 1, a observação será retirada de uma distribuição t de Student com média 0.1651 e desvio padrão 0.0256 e se estiver no segundo regime com média 0.0070 e desvio padrão 0.0199. A Figura 5.37 apresenta a probabilidade suavizada do regime 1 e os log-retornos. As probabilidades de estarmos no regime 1 variam entre 0 e 0.49 , as probabilidades neste caso oscilam bastante e as probabilidades de estarmos em um regime e continuarmos neste $p_{11}$ e $p_{22}$ são 0.4932 e 0.5391, respetivamente. Com isto temos que a matriz de transição e dada por

$$
P=\left(\begin{array}{ll}
0.4932 & 0.4609 \\
0.5068 & 0.5391
\end{array}\right) .
$$

Temos então que a probabilidade incondicional de estar no $k$-ésimo regime, neste caso para nosso modelo com 2 regimes é dada por $\pi_{1}=0.4762$ e $\pi_{2}=0.5238$. Ou seja, ao sortearmos uma observação qualquer da série, a probabilidade é maior de estarmos no regime 2 do que no regime 1.

A duração média de estarmos no regime 1 é 1 dia e do regime 2 é de 2 dias.
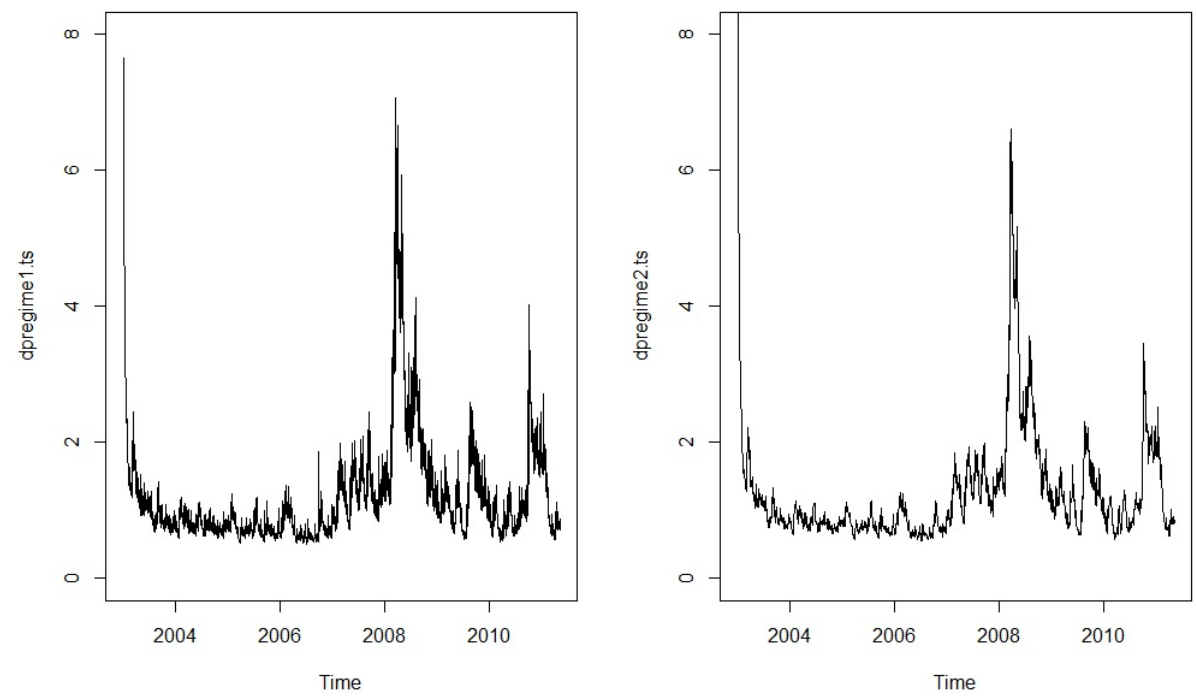

Figura 5.38: Estimativa do desvio padrão condicional para os retornos da SESP00 no Regime 1 e 2 com erro t de Student. 
As probabilidades suavizadas do regime 1 com erro GED e os log-retornos podem ser observados no seguinte gráfico:

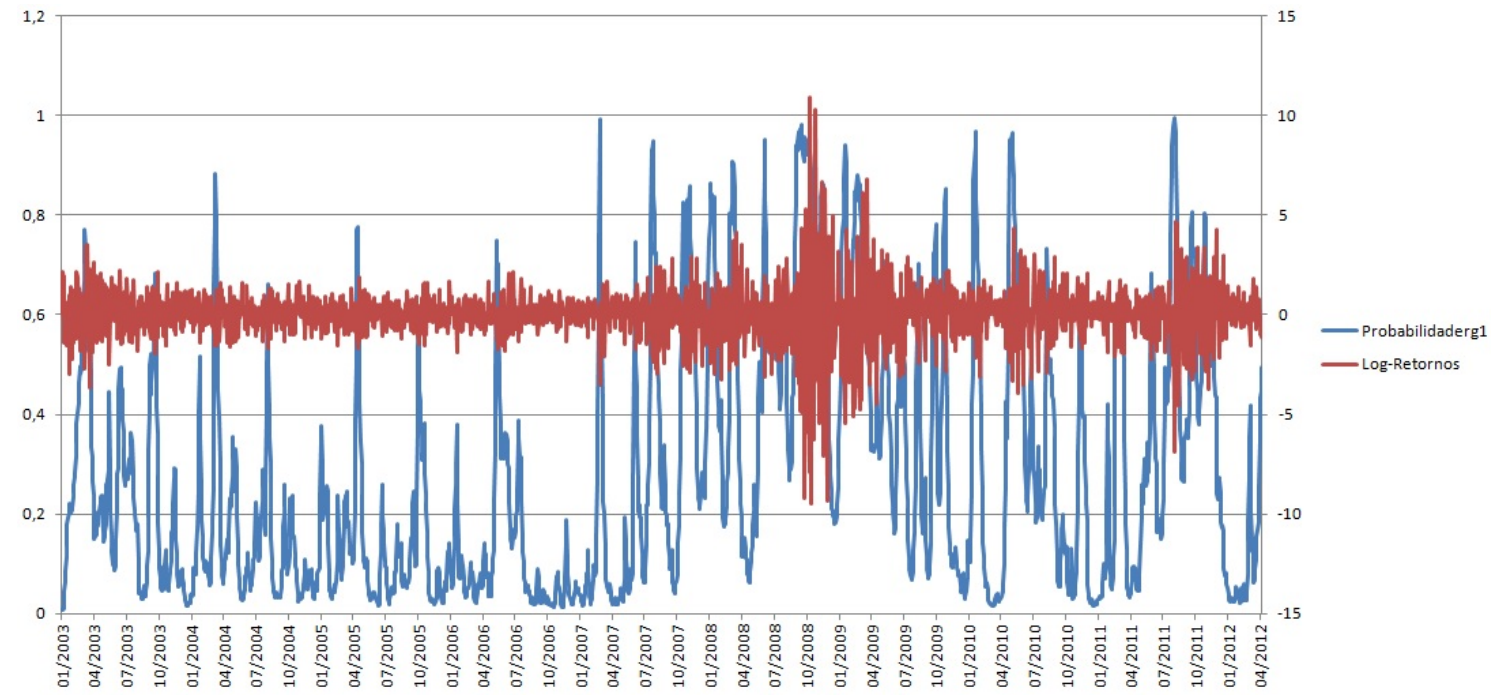

Figura 5.39: Probabilidade suavizada do Regime 1 - GED - S\&P500.

Se o processo estiver no regime 1, a observação será retirada de uma distribuição ged com média -0.2380 e desvio padrão 0.1370 e se estiver no segundo regime com média 0.1111 e desvio padrão 0.0224. A figura 5.39 apresenta a probabilidade suavizada do regime 1 e os log-retornos. As probabilidades de estarmos no regime 1 , variam entre 0 e 0.9 , parece haver períodos com menor volatidade como o ínicio da série até 2007, pode-se perceber que o gráfico das probabilidades suavizadas 5.35 e 5.39 tem uma performance semelhante. As probabilidades de estarmos em um regime e continuarmos neste $p_{11}$ e $p_{22}$ são 0.9088 e 0.9620 respetivamente. Com isto temos que a matriz de transição e dada por

$$
P=\left(\begin{array}{ll}
0.9088 & 0.0380 \\
0.0912 & 0.9620
\end{array}\right)
$$

Temos então que a probabilidade incondicional de estar no $k$-ésimo regime, neste caso para nosso modelo com 2 regimes é dada por $\pi_{1}=0.2941$ e $\pi_{2}=0.7059$. Ou seja, ao sortearmos uma observação qualquer da série, a probabilidade é maior de estarmos no regime 2 do que no regime 1.

A duração média de estarmos no regime 1 é 10 dias e do regime 2 é de 26 dias.
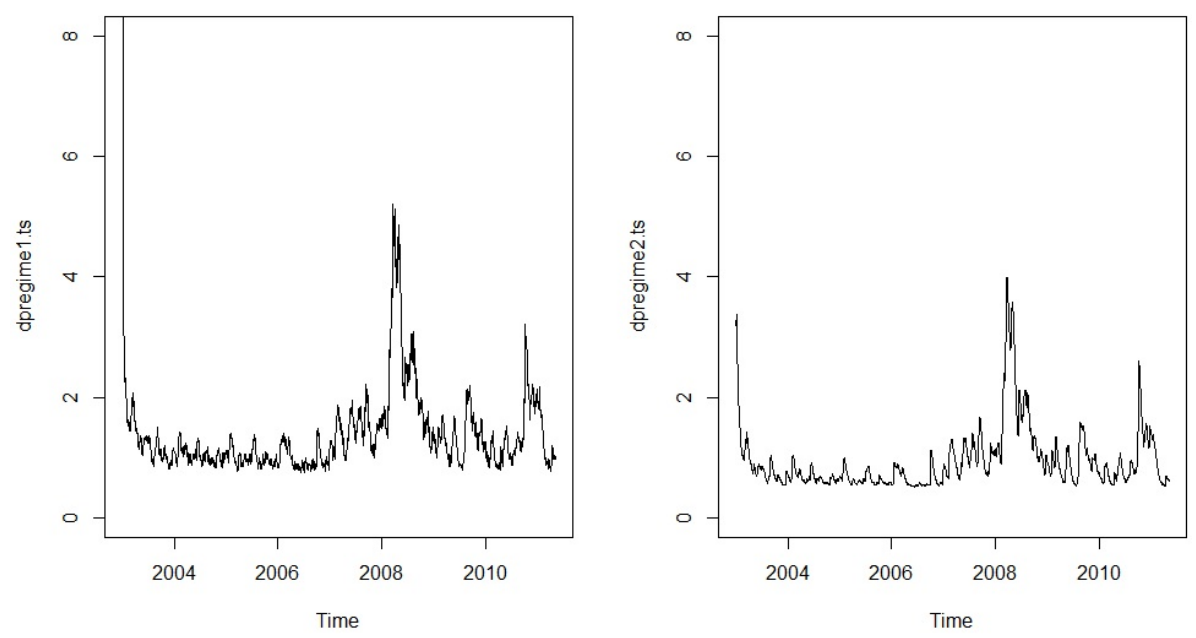

Figura 5.40: Estimativa do desvio padrão condicional para os retornos da SEPS00 no Regime 1 e 2 com erro GED. 


\subsection{Análise de Resíduos-S\&P500}

O modelo MRS-GARCH com $\epsilon_{t}$ normal, $t$-Student ou GED foi bem ajustado, se os resíduos padronizados

$$
\widetilde{r_{t}}=r_{t} / \sqrt{h_{t}}
$$

comportam-se como variáveis aleatorias i.i.d com distribuição normal padrão, $t$-Student ou GED. Como no caso de modelos GARCH, usualmente supomos que os $\epsilon_{t}$ são normais, ou seguem uma distribuição $t$-Student, ou ainda, uma distribuição de erro generalizada.

\subsubsection{Análise de Resíduos para o modelo MRS-GARCH-N}

Regime 1

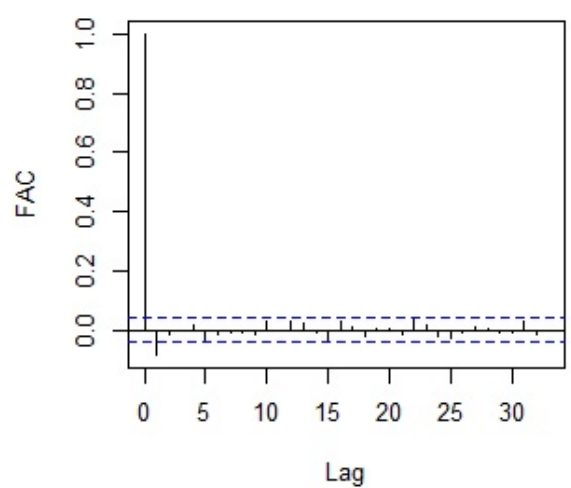

Regime 2

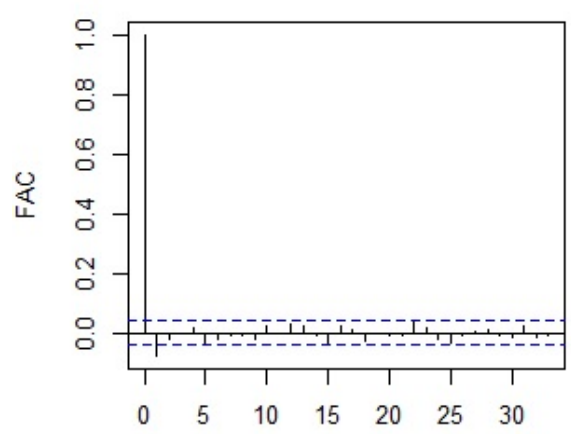

Lag
Regime 1

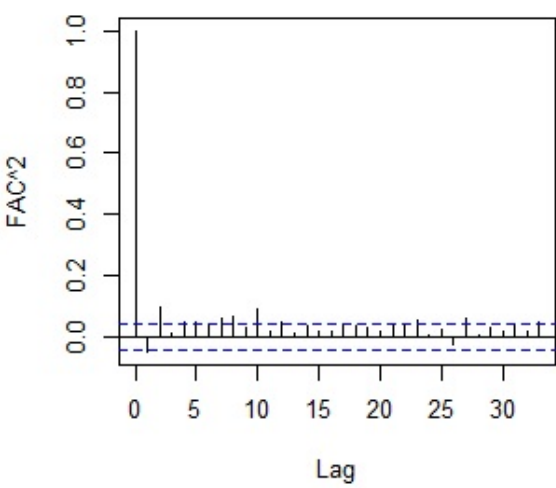

Regime 2

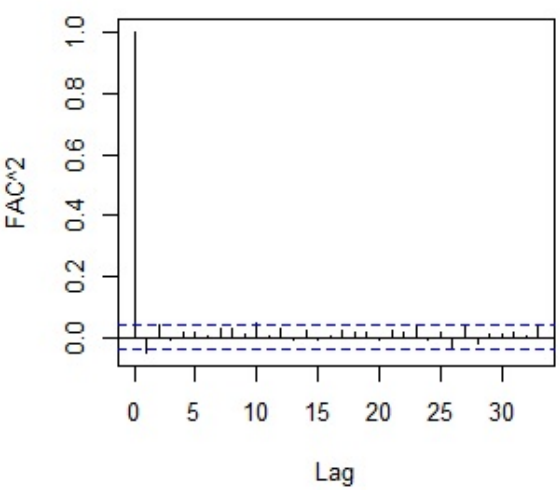

Figura 5.41: Função de autocorrelação e função de autocorrelação ao quadrado dos resíduos padronizados do modelo Normal-SEP 500. 

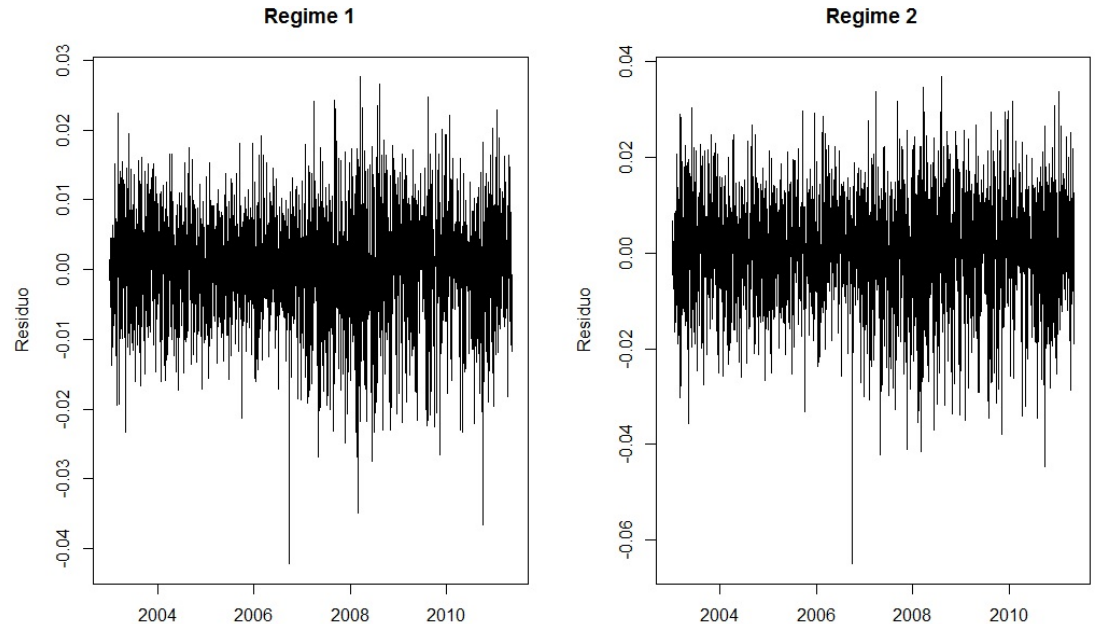

Figura 5.42: Resíduos padronizados nos Regimes 1 e 2 do modelo Normal-SESP500.
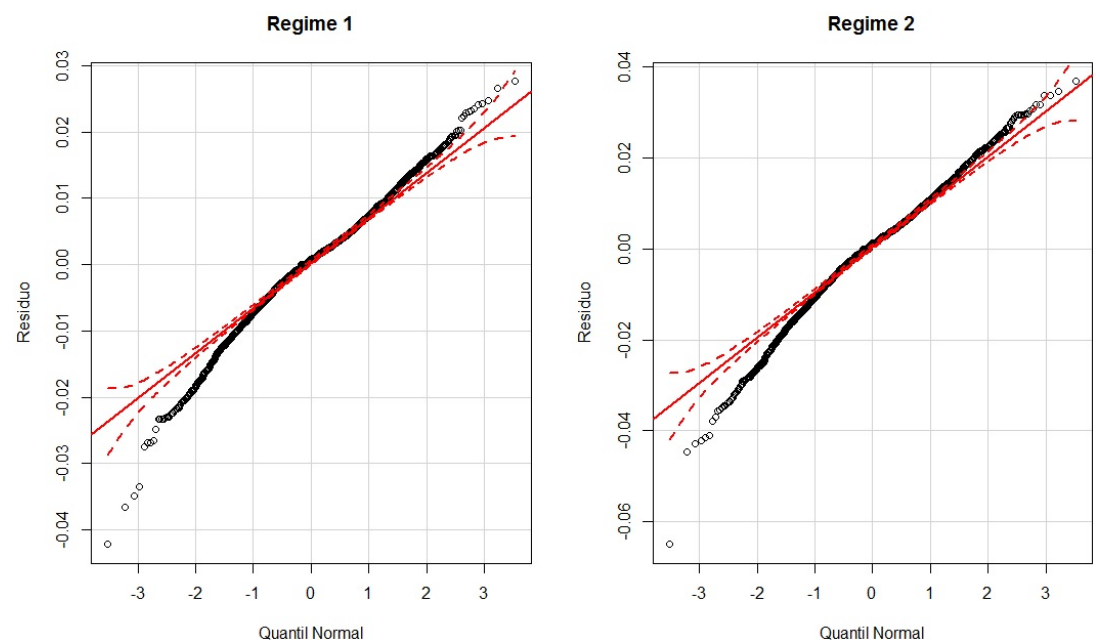

Figura 5.43: Gráfico de quantis dos Resíduos padronizados do modelo Normal-SEP500.

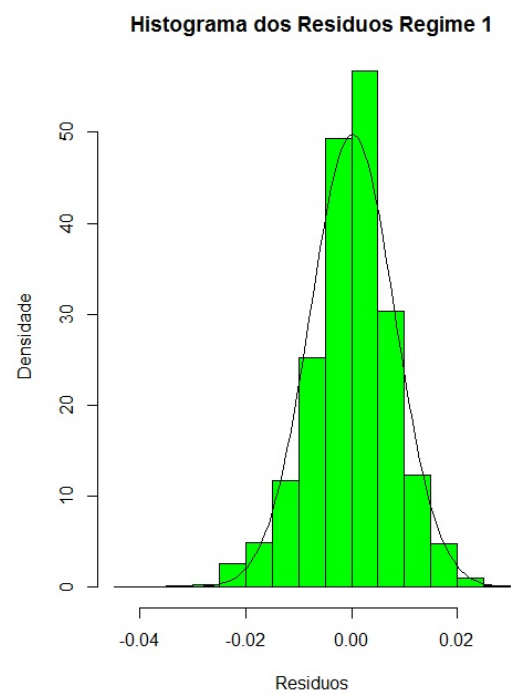

Histograma dos Residuos Regime 2

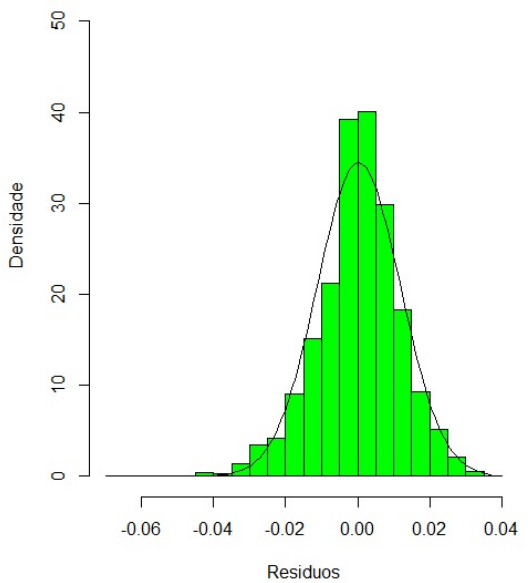

Figura 5.44: Histograma dos Resíduos padronizados do modelo Normal-SEP500. 


\subsubsection{Análise de Resíduos para o modelo MRS-GARCH-t2}
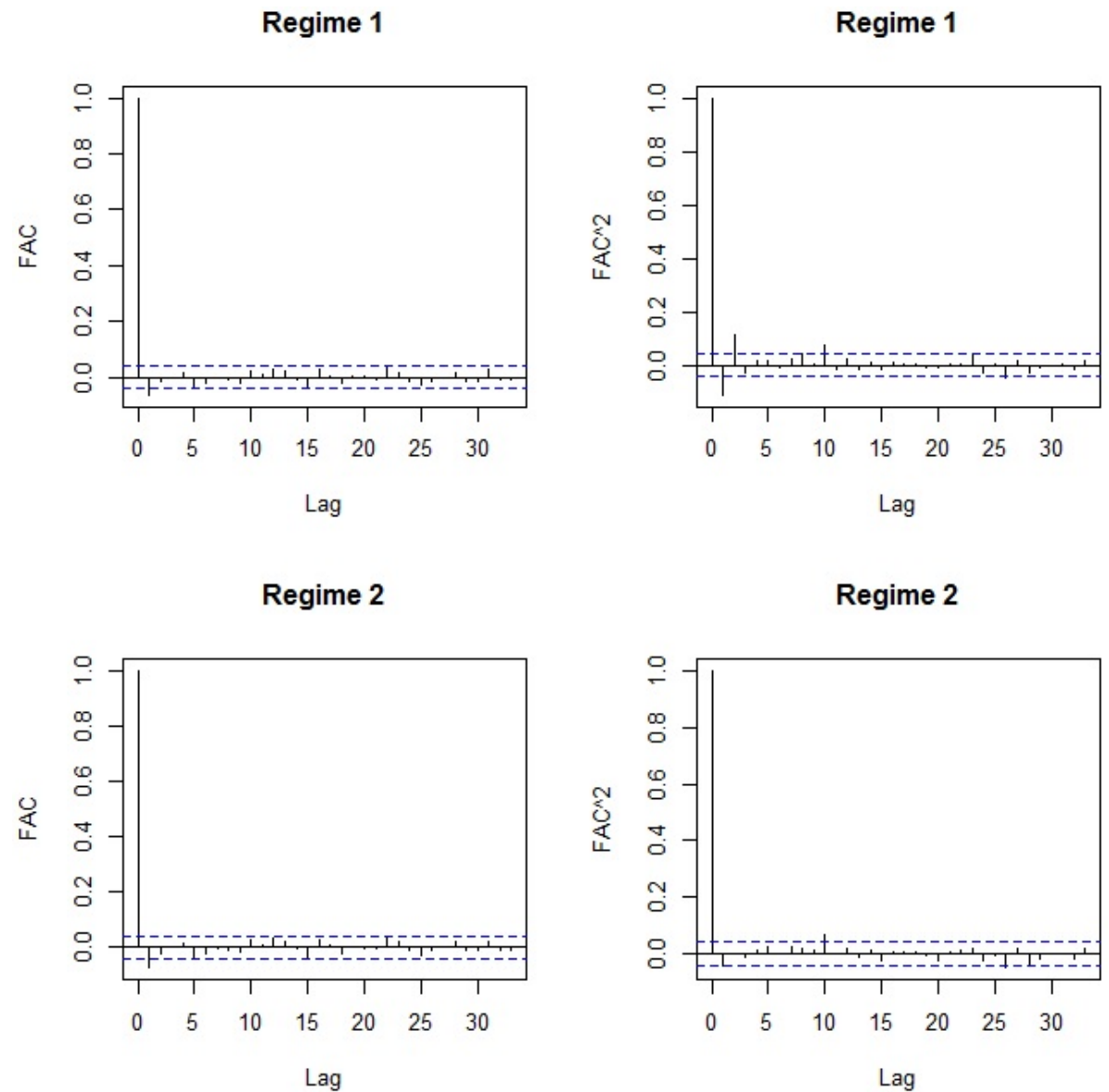

Figura 5.45: Função de autocorrelação e função de autocorrelação ao quadrado dos resíduos padronizados do modelo t2-SEP 500 .
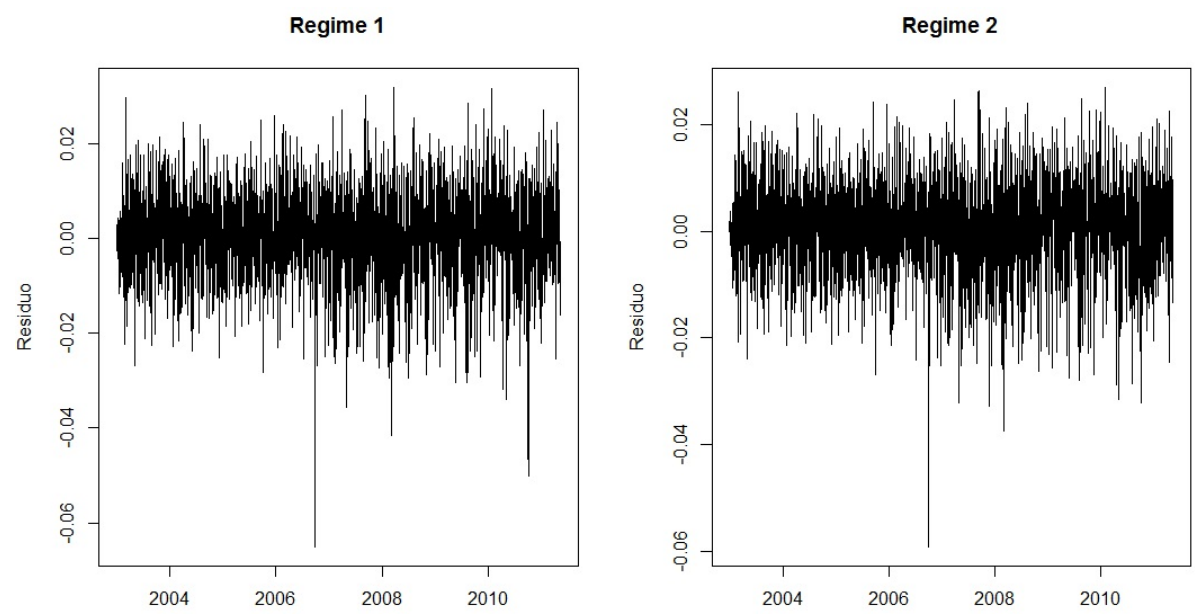

Figura 5.46: Resíduos padronizados nos Regimes 1 e 2 do modelo t2-SESP500. 

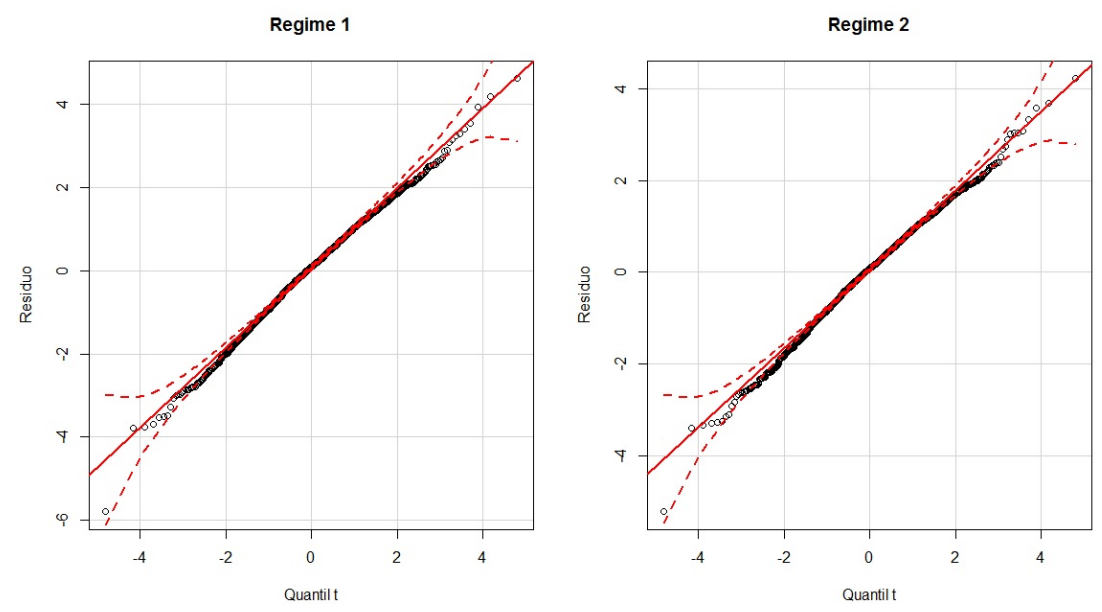

Figura 5.47: Gráfico de quantis dos Resíduos padronizados do modelo t2-S\&P500.

Histograma dos Residuos Regime 1

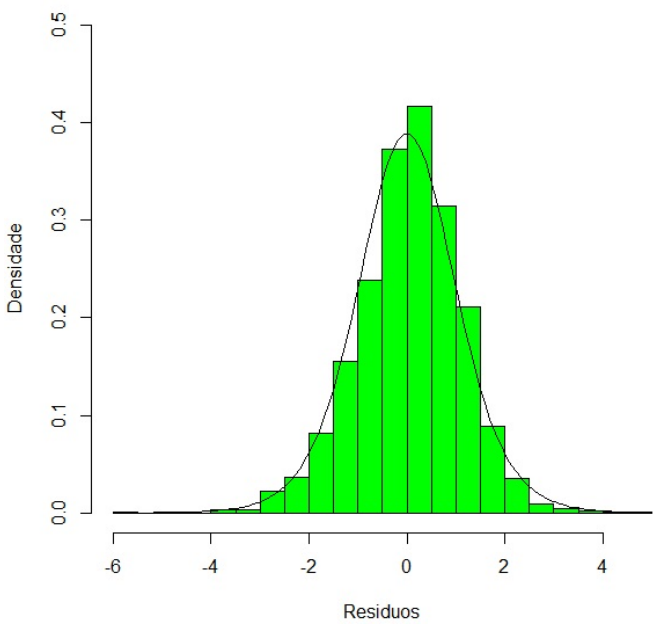

Histograma dos Residuos Regime 2

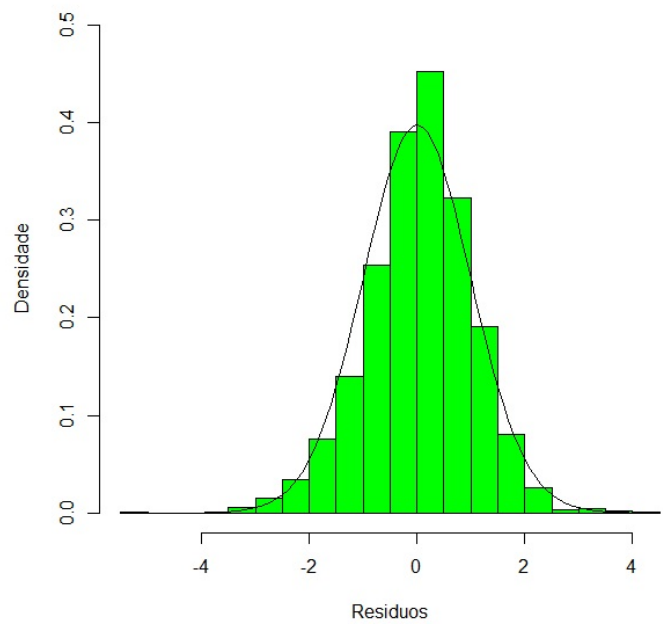

Figura 5.48: Histograma dos Resíduos padronizados do modelo t2-S\&P500. 


\subsubsection{Análise de Resíduos para o modelo MRS-GARCH-GED}

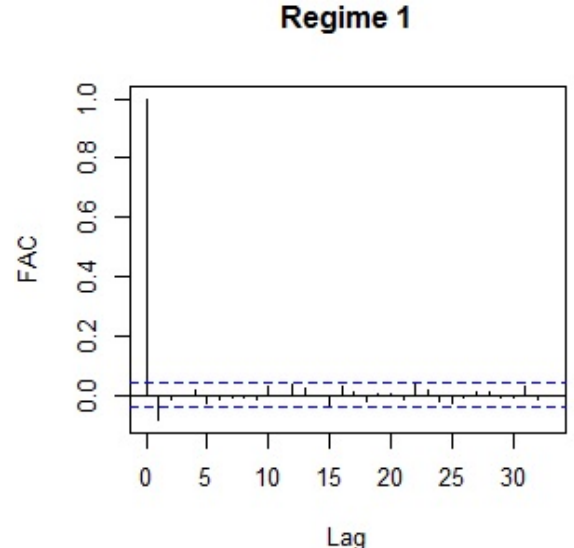

Regime 2

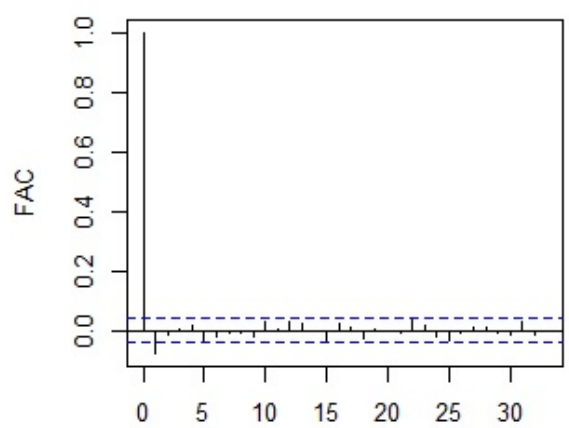

Lag
Regime 1

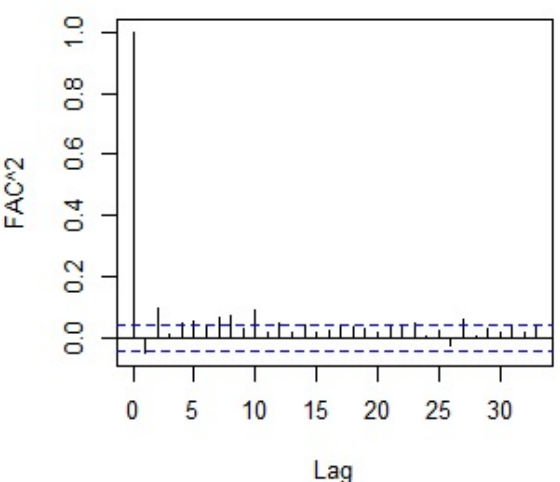

Regime 2

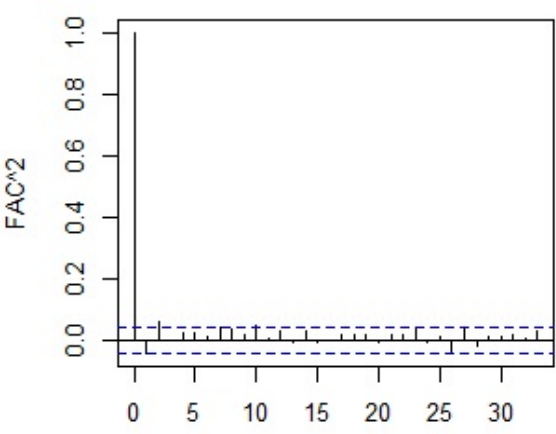

Lag

Figura 5.49: Função de autocorrelação e função de autocorrelação ao quadrado dos resíduos padronizados do modelo GED-SESP00.
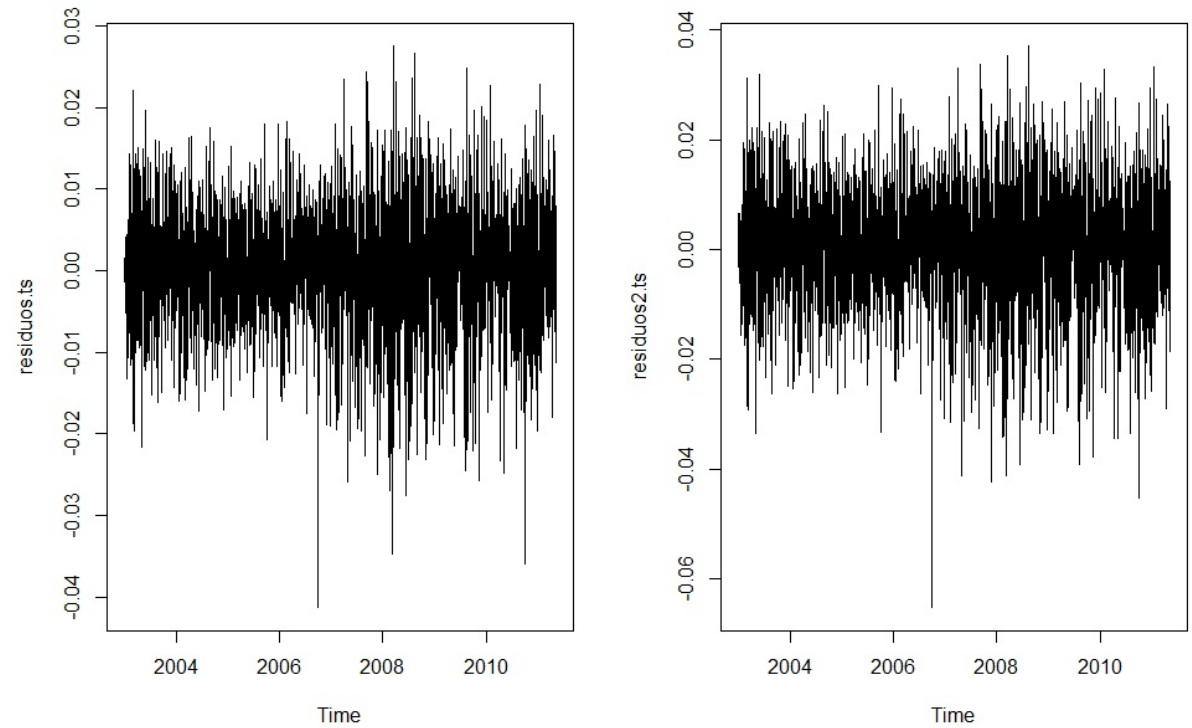

Figura 5.50: Resíduos padronizados nos Regimes 1 e 2 do modelo GED-SEP500. 

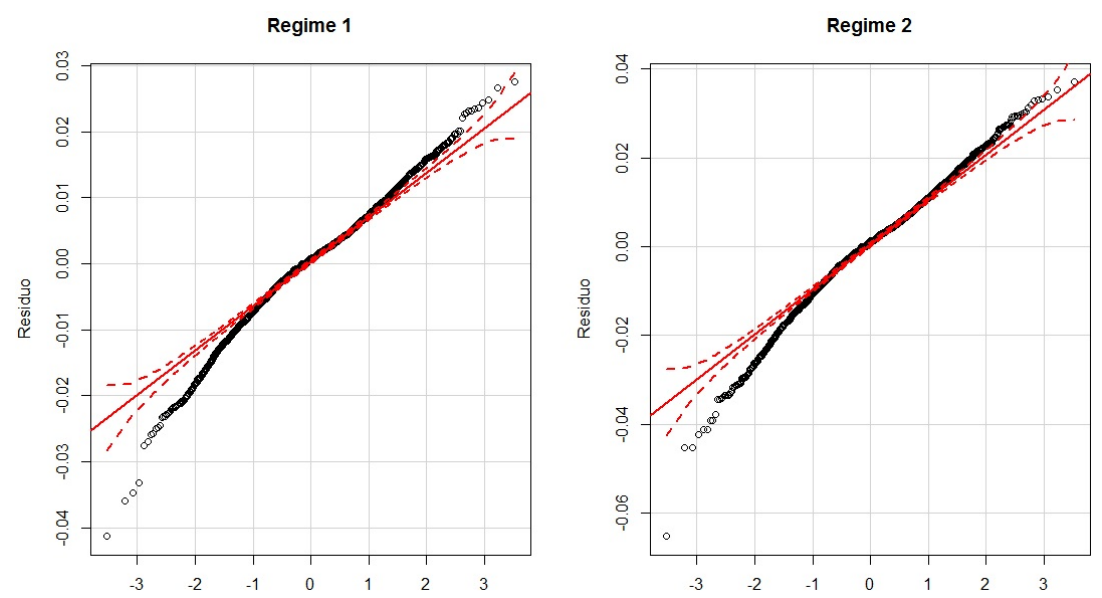

Figura 5.51: Gráfico de quantis dos Resíduos padronizados do modelo GED-SEP500.

Histograma dos Residuos Regime 1

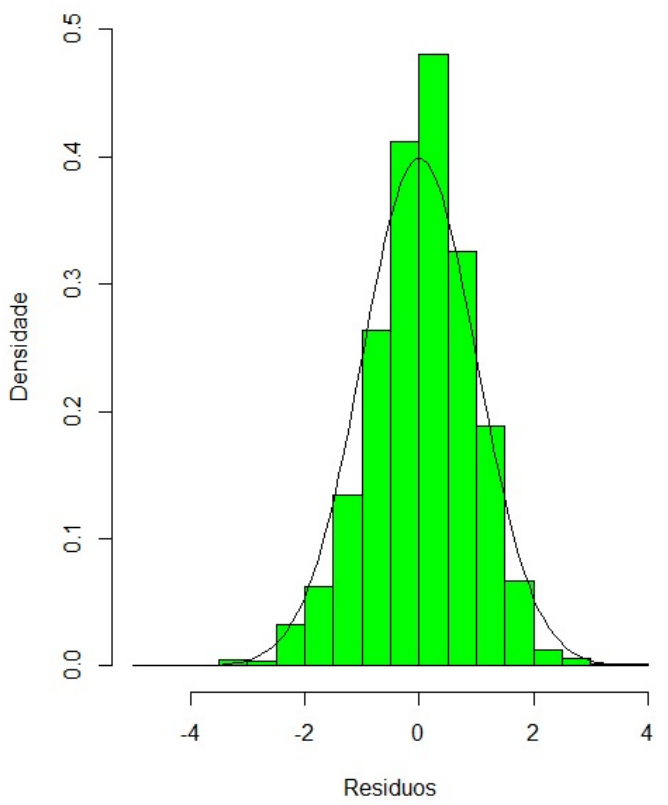

Histograma dos Residuos Regime 2

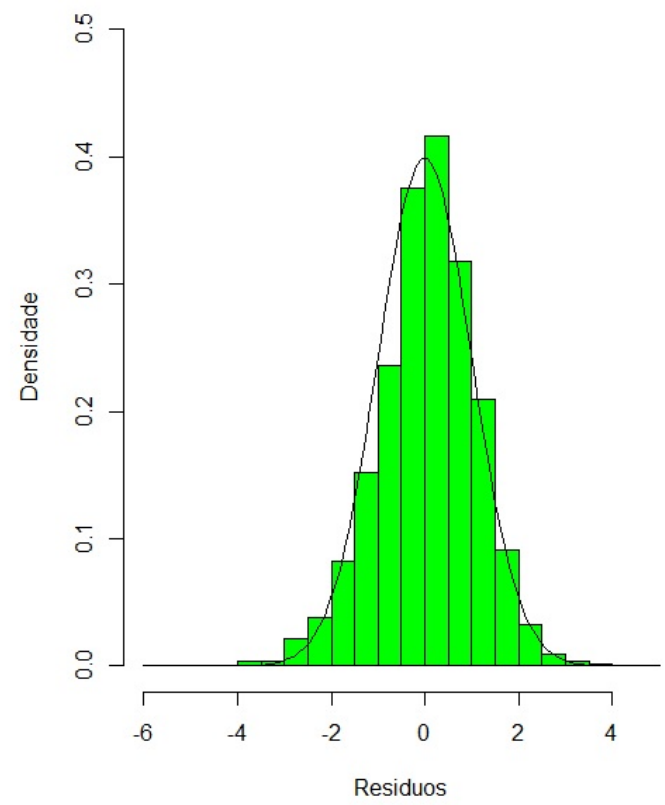

Figura 5.52: Histograma dos Resíduos padronizados do modelo GED-SEP500.

Os gráficos de quantis indicam que somente os modelos com distribuição t de Student com distintos graus de liberdade são adequados e por isso esse modelo foi escolhido para os log retornos da S\&P500. A função de autocorrelação parcial dos resíduos padronizados não indicam nenhuma quebra de comportamento de ruído branco nos resíduos. 
Em seguida, avaliaremos o desempenho dos modelos com base na cobertura dos intervalos de confiança para os log retornos.
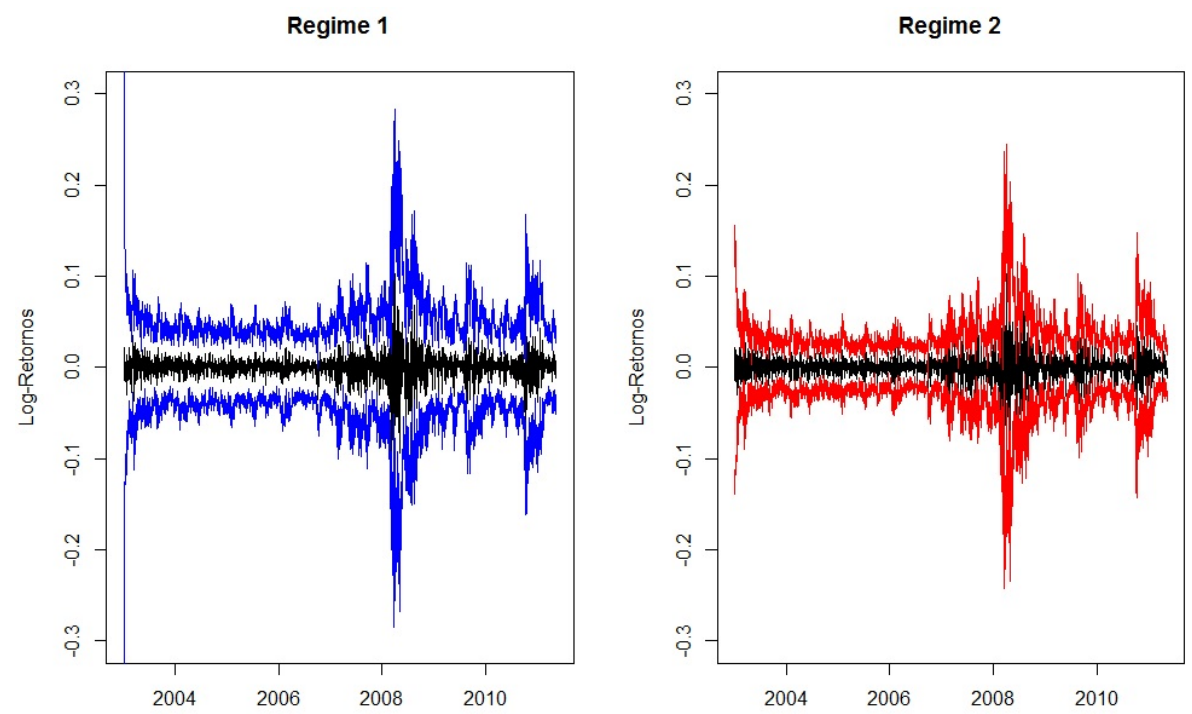

Figura 5.53: Retornos e Intervalos de confiança (95\%)-Normal-S\&PP00.
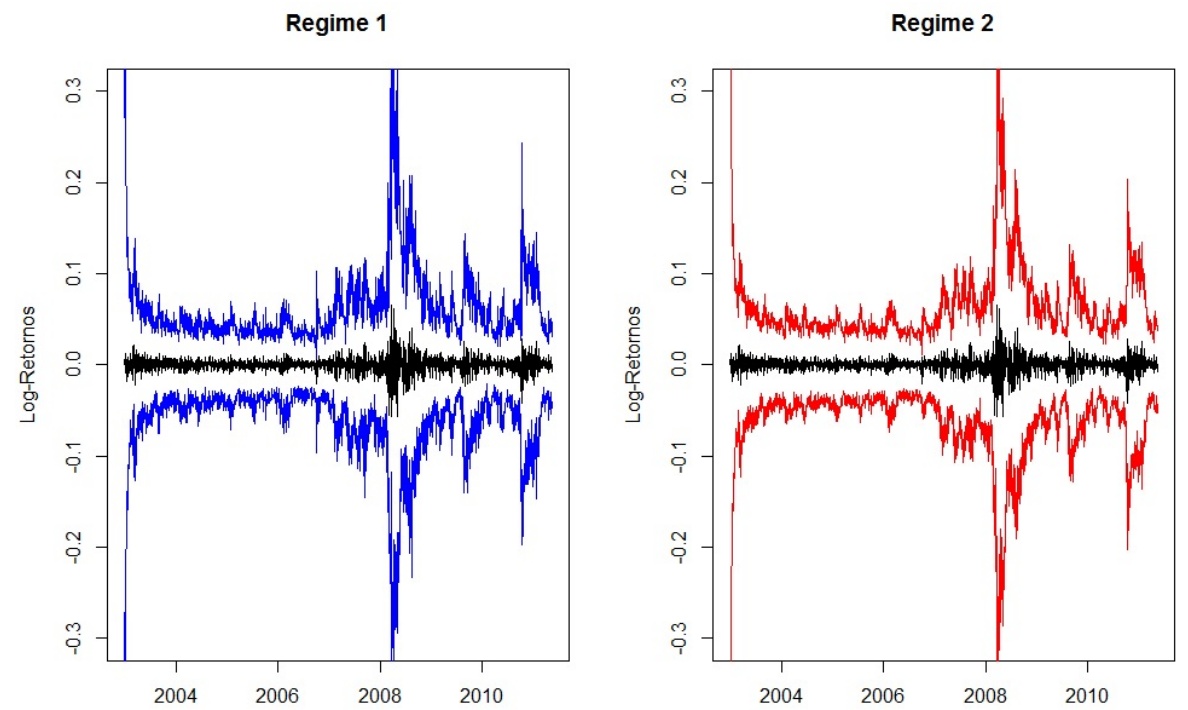

Figura 5.54: Retornos e Intervalos de confiança (95\%)-t2-S\&3P500. 

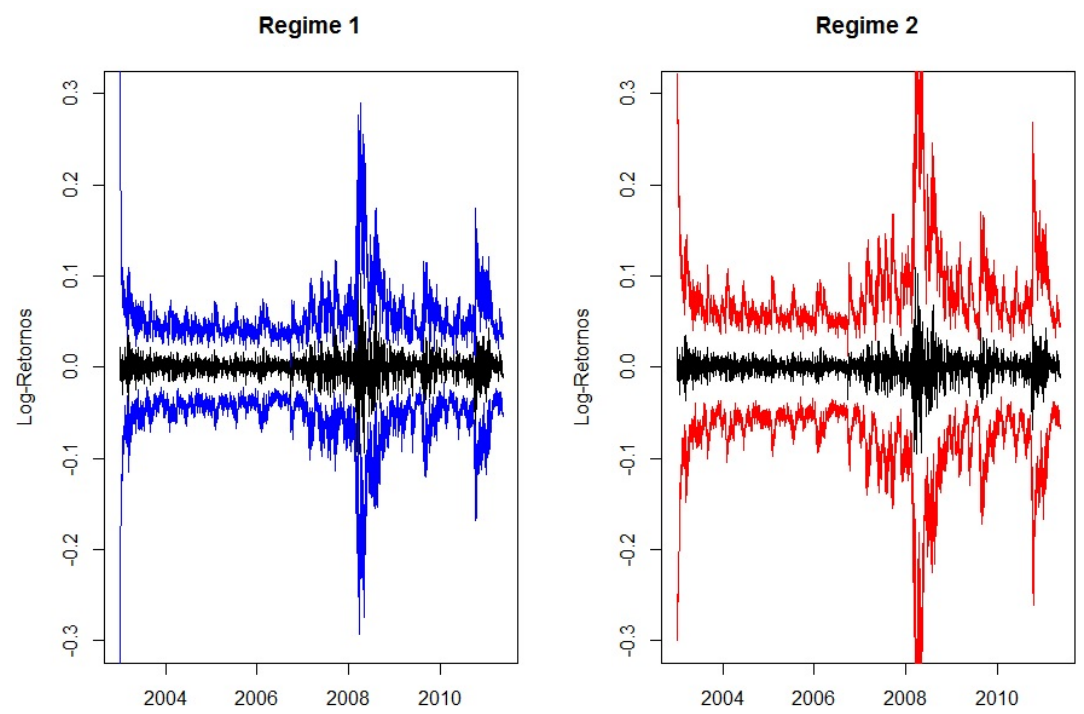

Figura 5.55: Retornos e Intervalos de confiança (95\%)-GED-SESP500.

Tabela 5.6: Porcentagem de Cobertura do intervalos de confiança para os log-retornos da SEPS00

\begin{tabular}{ccccc}
\hline & Cobertura (\% ) & Comp. Médio & Mínimo & Máximo \\
\hline GARCH(1,1)-N & 95 & 4.41 & 2.03 & 20.43 \\
2R.GARCH(1,1)-N & 99 & 5.40 & 3.00 & 79.90 \\
2R.GARCH(1,1)-t2 & 99 & 7.27 & 3.12 & 46.63 \\
GARCH(1,1)-GED & 98 & 5.26 & 2.81 & 24.01 \\
2R.GARCH(1,1)-GED & 99 & 5.63 & 3.02 & 79.18 \\
\hline
\end{tabular}

Na Tabela 5.6 mostra-se o modelo Garch usual como $\operatorname{GARCH}(1,1)$ para cada distribuição, e 2R.GARCH $(1,1)$ para o modelo Garch com dois regimes.

Os intervalos apresentaram cobertura maior que $95 \%$ e as maiores coberturas foram para as três distribuições utilizadas com dois regimes. Pode-se observar que os valores para os modelos com 2 regimes tem maior comprimento médio do que os modelos com 1 regime. 


\section{Capítulo 6}

\section{Conclusões}

Neste trabalho apresentamos os modelos GARCH e GARCH com mudança de regime para modelar a volatilidade de séries financeiras. Os modelos de volatilidade com mudança de regime apresentaram duas séries temporais não observadas, a série de volatilidade condicional e dos regimes. Podemos considerar as mudanças Markovianas de regime, que parece ser bastante razoável em situações práticas, já que em um regime hoje, deve influenciar o regime amanhã. Logo, o modelo GARCH com mudança de regime markoviana é bastante versátil para modelar comportamentos variados.

O objetivo principal deste trabalho foi pesquisar a eficácia do uso de modelos com mudança de regime Markoviano para aplicá-los às séries financeiras. Cabe ressaltar o fato de que estas séries, Ibovespa e S\&P500, foram muito influenciadas pela crise financeira do subprime em 2008, que mudaram bastante a dinâmica destas séries imediatamente após a crise mencionada. Os modelos que analisamos neste trabalho conseguiram detectar um regime com maior efeito do erro passado e outro com maior inércia para a variância anterior.

Além disso, estes modelos fornecem-nos informações úteis a respeito dos parâmetros de cada uma das fases. Os modelos com mudança de regime permitiram modelar séries que apresentaram mudanças em seus parâmetros e estimar probabilidades de cada regime ao longo do tempo.

$\mathrm{Na}$ literatura tem-se mostrado que os modelos com mudança de regime markoviano para séries financeiras com distribuição t de Student se ajustam bem as séries. Aqui neste trabalho pode-se observar que o modelo com distribuição t de Student com distintos graus de liberdade conseguiu corrigir a dependência da volatilidade (ver plot dos resíduos para cada distribuição do erro), o que quer dizer que o modelo proposto se ajustou bem às séries. Também as funções de autocorrelação e autocorrelação parcial dos resíduos padronizados e resíduos padronizados ao quadrado não indicam nenhuma quebra de comportamento de ruído branco nos resíduos.

Em ambas aplicações, para os log-retornos do Ibovespa e do S\&P500, o modelo GARCH $(1,1)$ com mudança de regime e erros com distribuição t de Student com graus de liberdade distintos apresentou bom ajuste e todas as suposições do modelo parecem satisfeitas. Entretanto, observando as probabilidades estimadas (suavizadas) dos regimes, não é possível identificar períodos em que o regime 1 deve prevalecer ou o regime 2. Além disso, as séries dos desvios padrões condicionais estimadas são muito semelhantes, o que se torna a distinção entre os regimes um tanto quanto confusa. Essa semelhança entre os regime não parece ocorrer ao analisarmos séries de atividade econômica como em Alencar (2013) e Correa (2004), nesses trabalhos é possível identificar claramente os períodos de aceleração e recessão econômica e os modelos com mudança de regime são utilizados para tal identificação.

Apesar da distribuição normal e GED não apresentarem bom ajuste ao considerarmos os gráficos de quantis dos resíduos padronizados de cada regime, para os retornos do S\&P500 as probabilidades 
suavizadas parecem identificar mais claramente períodos em que ocorrem cada regime.

Através da análise de resíduos do modelo GARCH com mudança de regime verificou-se que a distribuição t-Student com distintos graus de liberdade se mostrou superior a distribuição normal e GED para caracterizar a distribuição dos retornos. A porcentagem de cobertura dos intervalos de confiança para a distribuição normal do modelo GARCH com mudança de regime foi superior em relação ao modelo GARCH usual, o mesmo aconteceu para a distribuições t-Student com distintos graus de liberdade, o que pode-se tornar interessante sua utilização no mercado financeiro.

A maior conclusão e contribuição desta dissertação é a ótima performance dos modelos de mudanca de regime tanto para captar a dinâmica das séries em análise quanto para a realização de regras de mercado. A aplicação destes modelos ao mercado financeiro tem um grande campo de estudo que com certeza ira se difundir muito no futuro próximo. 


\section{Apêndice A}

\section{Mistura de Distribuições}

Mistura de Normais A densidade de $y_{t} / s_{t}=j$ é dada por

$$
f\left(y_{t} / s_{t}=j ; \theta\right)=\frac{1}{\sqrt{2 \pi} \sigma_{j}} \exp \left\{-\frac{\left(y_{t}-\mu_{j}\right)^{2}}{2 \sigma_{j}^{2}}\right\},
$$

em que $j=1,2, \ldots, k$ e $\theta$ é o vetor de parâmetros, $\mu_{s_{t}}$ e $\sigma_{s t}^{2}$ são dados segundo o valor da variável estado da natureza.

Por Bayes

$$
p\left(y_{t}, s_{t}=j ; \theta / Y_{t}\right)=f\left(y_{t} / s_{t}=j ; \theta\right) p\left(s_{t}=j ; \theta\right)
$$

onde $P\left(s_{t}=j ; \theta\right)=\pi_{j}$

$$
p\left(y_{t}, s_{t}=j ; \theta / Y_{t}\right)=\frac{\pi_{j}}{\sqrt{2 \pi} \sigma_{j}} \exp \left\{-\frac{\left(y_{t}-\mu_{j}\right)^{2}}{2 \sigma_{j}^{2}}\right\}
$$

A distribuição não condicional de $y_{t}$ será dada pela soma das probabilidades ergodicas em cada regime

$$
f\left(y_{t} ; \theta\right)=\sum_{j=1}^{k} p\left(y_{t}, s_{t}=j ; \theta / Y_{t}\right)
$$

Neste caso temos dois regimes, logo temos

$$
f\left(y_{t} ; \theta\right)=\frac{\pi_{1}}{\sqrt{2 \pi} \sigma_{1}} \exp \left\{-\frac{\left(y_{t}-\mu_{1}\right)^{2}}{2 \sigma_{1}^{2}}\right\}+\frac{\pi_{2}}{\sqrt{2 \pi} \sigma_{2}} \exp \left\{-\frac{\left(y_{t}-\mu_{2}\right)^{2}}{2 \sigma_{2}^{2}}\right\}
$$

A soma de duas normais tem como distribuição

$$
r_{t}^{(1)} \pi_{1}+r_{t}^{(2)} \pi_{2} \rightarrow \text { MisturadeNormais }\left(\begin{array}{c}
\mu_{1} \\
\mu_{2}
\end{array}\right)\left(\begin{array}{l}
\pi_{1} \\
\pi_{2}
\end{array}\right)\left(\begin{array}{c}
\sigma_{1}^{2} \\
\sigma_{2}^{2}
\end{array}\right)
$$

Vamos a encontrar sua média e sua variância respetivamente. Então,

$$
\begin{gathered}
\mu=E\left[r_{t}^{(1)} \pi_{1}+r_{t}^{(2)} \pi_{2}\right]=\pi 1 E\left[r_{t}^{(1)}\right]+\pi_{2} E\left[r_{t}^{(2)}\right]=\pi_{1} \mu_{1}+\pi_{2} \mu_{2}=\pi_{1} \mu_{1}+\left(1-\pi_{1}\right) \mu_{2} \\
V a r=V\left[r_{t}^{(1)} \pi_{1}+r_{t}^{(2)} \pi_{2}\right]=\pi 1^{2} V\left[r_{t}^{(1)}\right]+\pi_{2}^{2} V\left[r_{t}^{(2)}\right]=\pi_{1}^{2} \sigma_{1}^{2}+\pi_{2}^{2} \sigma_{2}^{2}=\pi_{1}^{2} \sigma_{1}^{2}+\left(1-\pi_{1}\right)^{2} \sigma_{2}^{2}
\end{gathered}
$$


Logo,

$$
r_{t}^{(1)} \pi_{1}+r_{t}^{(2)} \pi_{2} \rightarrow M N\left(\pi_{1} \mu_{1}+\pi_{2} \mu_{2}, \pi_{1}^{2} \sigma_{1}^{2}+\pi_{2}^{2} \sigma_{2}^{2}\right)
$$

Mistura de t-Student

$$
Q(\theta)=\sum_{h=1}^{H} p_{h} t_{d}\left(\theta / \mu_{h}, \sum_{h}, \nu\right)
$$

onde $p_{h},(h=1, \ldots, H)$ são as probabilidades de mistura de componentes t-Student, $0 \leq p_{h} \leq$ $1, \sum_{h=1}^{H} p_{h}=1$, e $t_{d}\left(\theta / \mu_{h}, \sum_{h}, \nu\right)$ é a densidade d-dimensional t-Student com vetor de modo $\mu_{h}$, matriz escala $\sum_{h}$, e $\nu$ graus de liberdade.

$$
t_{d}\left(\theta / \mu_{h}, \sum_{h}, \nu\right)=\frac{\Gamma\left(\frac{\nu+d}{2}\right)}{\Gamma\left(\frac{\nu}{2}\right)(\pi \nu)^{d / 2}} \times\left(\operatorname{det} \sum_{h}\right)^{-1 / 2}\left(1+\frac{\left(\theta-\mu_{h}\right)^{\prime} \sum_{h}^{-1}\left(\theta-\mu_{h}\right)}{\nu}\right)^{\frac{-(\nu+d)}{2}}
$$

Nosso caso a distribuição t-Student de nosso interesse tem uma dimensão. Então com $d=1, \sum_{h}=$ $\sigma_{h}^{2}$ e $\theta=x$ temos que:

$$
t_{d}\left(\theta / \mu_{h}, \sum_{h}, \nu\right)=\frac{\Gamma\left(\frac{\nu+1}{2}\right)}{\Gamma\left(\frac{\nu}{2}\right)(\pi \nu)^{1 / 2}} \frac{1}{\sigma_{h}}\left(1+\frac{\left(x-\mu_{h}\right)^{2}}{\nu \sigma_{h}^{2}}\right)^{\frac{-(\nu+1)}{2}}
$$

Para cada regime temos as seguintes funções de distribuição,

$$
\begin{aligned}
& t_{1}=\frac{\Gamma\left(\frac{\nu_{1}+1}{2}\right)}{\Gamma\left(\frac{\nu_{1}}{2}\right)\left(\pi \nu_{1}\right)^{1 / 2}} \frac{1}{\sigma_{1}}\left(1+\frac{\left(x-\mu_{1}\right)^{2}}{\nu_{1} \sigma_{1}^{2}}\right)^{\frac{-\left(\nu_{1}+1\right)}{2}} \\
& t_{2}=\frac{\Gamma\left(\frac{\nu_{2}+1}{2}\right)}{\Gamma\left(\frac{\nu_{2}}{2}\right)\left(\pi \nu_{2}\right)^{1 / 2}} \frac{1}{\sigma_{2}}\left(1+\frac{\left(x-\mu_{2}\right)^{2}}{\nu_{2} \sigma_{2}^{2}}\right)^{\frac{-\left(\nu_{2}+1\right)}{2}}
\end{aligned}
$$

$\log$,

$$
\begin{aligned}
q(\theta) & =p_{1} t_{1}+p_{2} t_{2} \\
& =\frac{p_{1} \Gamma\left(\frac{\nu_{1}+1}{2}\right)}{\Gamma\left(\frac{\nu_{1}}{2}\right)\left(\pi \nu_{1}\right)^{1 / 2}} \frac{1}{\sigma_{1}}\left(1+\frac{\left(x-\mu_{1}\right)^{2}}{\nu_{1} \sigma_{1}^{2}}\right)^{\frac{-\left(\nu_{1}+1\right)}{2}}+\frac{\left(1-p_{1}\right) \Gamma\left(\frac{\nu_{2}+1}{2}\right)}{\Gamma\left(\frac{\nu_{2}}{2}\right)\left(\pi \nu_{2}\right)^{1 / 2}} \frac{1}{\sigma_{2}}\left(1+\frac{\left(x-\mu_{2}\right)^{2}}{\nu_{2} \sigma_{2}^{2}}\right)^{\frac{-\left(\nu_{2}+1\right)}{2}}
\end{aligned}
$$

Então temos,

$$
T=\begin{aligned}
& T_{1} \\
& T_{2}
\end{aligned} \rightarrow T_{2, \nu_{1}, \nu_{2}}\left(\mu_{1}, \mu_{2}, \sum_{h}\right)
$$

Para saber a forma fechada da densidade $q(\theta)$ temos que fazer $x=p_{1} t_{1}$ e $y=p_{2} t_{2}$. Chamemos $z=x+y$.

$$
p(Z \leq z)=p(x+y \leq z)=\int_{x=0}^{x=z} \int_{y=0}^{y=z-x} f(x, y) d x d y
$$


Primeiramente vamos calcular a $f(x, y)$.

$$
\begin{aligned}
f(x, y) & =\frac{\Gamma\left(\frac{\nu_{1}+1}{2}\right) p_{1}}{\Gamma\left(\frac{\nu_{1}}{2}\right)\left(\pi \nu_{1}\right)^{1 / 2}} \frac{1}{\sigma_{1}}\left(1+\frac{\left(x-\mu_{1}\right)^{2}}{\nu_{1} \sigma_{1}^{2}}\right)^{\frac{-\left(\nu_{1}+1\right)}{2}} \times \frac{\Gamma\left(\frac{\nu_{2}+1}{2}\right)\left(1-p_{1}\right)}{\Gamma\left(\frac{\nu_{2}}{2}\right)\left(\pi \nu_{2}\right)^{1 / 2}} \frac{1}{\sigma_{2}}\left(1+\frac{\left(x-\mu_{2}\right)^{2}}{\nu_{2} \sigma_{2}^{2}}\right)^{\frac{-\left(\nu_{2}+1\right)}{2}} \\
& =\frac{\Gamma\left(\frac{\nu_{1}+1}{2}\right) \Gamma\left(\frac{\nu_{2}+1}{2}\right) p_{1}\left(1-p_{1}\right)}{\Gamma\left(\frac{\nu_{1}}{2}\right) \Gamma\left(\frac{\nu_{2}}{2}\right)\left(\pi \nu_{1}\right)^{1 / 2}\left(\pi \nu_{2}\right)^{1 / 2}} \frac{1}{\sigma_{1} \sigma_{2}}\left(1+\frac{\left(x-\mu_{1}\right)^{2}}{\nu_{1} \sigma_{1}^{2}}\right)^{\frac{-\left(\nu_{1}+1\right)}{2}}\left(1+\frac{\left(x-\mu_{2}\right)^{2}}{\nu_{2} \sigma_{2}^{2}}\right)^{\frac{-\left(\nu_{2}+1\right)}{2}} \text { (A.2) }
\end{aligned}
$$

Substituindo A2 em A1

$$
\begin{gathered}
=\frac{\Gamma\left(\frac{\nu_{1}+1}{2}\right) \Gamma\left(\frac{\nu_{2}+1}{2}\right) p_{1}\left(1-p_{1}\right)}{\Gamma\left(\frac{\nu_{1}}{2}\right) \Gamma\left(\frac{\nu_{2}}{2}\right)\left(\pi \nu_{1}\right)^{1 / 2}\left(\pi \nu_{2}\right)^{1 / 2}} \frac{1}{\sigma_{1} \sigma_{2}} \int_{x=0}^{x=z} \int_{y=0}^{y=z-x}\left(1+\frac{\left(x-\mu_{1}\right)^{2}}{\nu_{1} \sigma_{1}^{2}}\right)^{\frac{-\left(\nu_{1}+1\right)}{2}} \\
\left(1+\frac{\left(x-\mu_{2}\right)^{2}}{\nu_{2} \sigma_{2}^{2}}\right)^{\frac{-\left(\nu_{2}+1\right)}{2}} d y d x \\
=\frac{\Gamma\left(\frac{\nu_{1}+1}{2}\right) \Gamma\left(\frac{\nu_{2}+1}{2}\right) p_{1}\left(1-p_{1}\right)}{\Gamma\left(\frac{\nu_{1}}{2}\right) \Gamma\left(\frac{\nu_{2}}{2}\right)\left(\pi \nu_{1}\right)^{1 / 2}\left(\pi \nu_{2}\right)^{1 / 2}} \frac{1}{\sigma_{1} \sigma_{2}} \int_{x=0}^{x=z}\left(1+\frac{\left(x-\mu_{1}\right)^{2}}{\nu_{1} \sigma_{1}^{2}}\right)^{\frac{-\left(\nu_{1}+1\right)}{2}} \\
{\left[\int_{y=0}^{y=z-x}\left(1+\frac{\left(x-\mu_{2}\right)^{2}}{\nu_{2} \sigma_{2}^{2}}\right)^{\frac{-\left(\nu_{2}+1\right)}{2}} d y\right] d x}
\end{gathered}
$$

Vamos a calcular

$$
\int_{y=0}^{y=z-x}\left(1+\frac{\left(x-\mu_{2}\right)^{2}}{\nu_{2} \sigma_{2}^{2}}\right)^{\frac{-\left(\nu_{2}+1\right)}{2}} d y
$$

Defina $c=\frac{\nu+1}{2}, b=\nu_{2} \sigma_{2}^{2}, b>0 e a=\mu_{2}$ Logo,

$$
\int_{y=0}^{y=z-x}\left(1+\frac{(y-a)^{2}}{b}\right)^{-c} d y
$$

Encontraremos uma primitiva que tenha forma fechada,

$$
\int\left(1+\frac{(y-a)^{2}}{b}\right)^{-c} d y
$$

Defina, $\frac{y-a}{\sqrt{b}}=w \Rightarrow \frac{d y}{\sqrt{b}}=d w$, Então

$$
\begin{array}{r}
\int\left(1+\frac{(y-a)^{2}}{b}\right)^{-c} d y=\int\left(1+w^{2}\right)^{-c} \sqrt{b} d w=\sqrt{b} \int \frac{1}{\left(1+w^{2}\right)^{c}} d w \\
=\sqrt{b}\left[w 2 F_{1}\left(\frac{1}{2}, c ; \frac{3}{2} ;-w^{2}\right)+c t e\right] \\
=\sqrt{b}\left[\frac{y-a}{\sqrt{b}} 2 F_{1}\left(\frac{1}{2}, \frac{\nu_{2}+1}{2} ; \frac{3}{2} ;-\frac{(y-a)^{2}}{b}\right)+c t e\right] \\
=(y-a) 2 F_{1}\left(\frac{1}{2}, \frac{\nu_{2}+1}{2} ; \frac{3}{2} ;-\frac{(y-a)^{2}}{b}\right)+c t e
\end{array}
$$




$$
=\left(y-\mu_{2}\right) 2 F_{1}\left(\frac{1}{2}, \frac{\nu_{2}+1}{2} ; \frac{3}{2} ;-\frac{\left(y-\mu_{2}\right)^{2}}{b}\right)+c t e
$$

Então,

$$
\begin{gathered}
\int_{y=0}^{y=z-x}\left(1+\frac{(y-a)^{2}}{b}\right)^{-c} d y=\left.\left(y-\mu_{2}\right) 2 F_{1}\left(\frac{1}{2}, \frac{\nu_{2}+1}{2} ; \frac{3}{2} ;-\frac{\left(y-\mu_{2}\right)^{2}}{b}\right)\right|_{y=0} ^{y=z-x} \\
=\left(z-x-\mu_{2}\right) 2 F_{1}\left(\frac{1}{2}, \frac{\nu_{2}+1}{2} ; \frac{3}{2} ;-\frac{\left(z-x-\mu_{2}\right)^{2}}{b}\right)-\left(-\mu_{2}\right) 2 F_{1}\left(\frac{1}{2}, \frac{\nu_{2}+1}{2} ; \frac{3}{2} ;-\frac{\left(-\mu_{2}\right)^{2}}{b}\right)
\end{gathered}
$$

Definamos agora,

$$
2 F_{1}(a, b ; c ; z)=\frac{\Gamma(c)}{\Gamma(b) \Gamma(c-b)} \int_{0}^{1} \frac{t^{b-1}(1-t)^{c-b-1}}{(1-t z)^{a}} d t
$$

Em nosso caso,

$$
\begin{aligned}
& 2 F_{1}\left(\frac{1}{2}, \frac{\nu_{2}+1}{2} ; \frac{3}{2} ;-\frac{\left(z-x-\mu_{2}\right)^{2}}{\nu_{2} \sigma_{2}^{2}}\right)=\frac{\Gamma\left(\frac{3}{2}\right)}{\Gamma\left(\frac{\nu_{2}+1}{2}\right) \Gamma\left(\frac{3}{2}-\frac{\nu_{2}+1}{2}\right)} \int_{0}^{1} \frac{t^{\frac{\nu_{2}+1}{2}-1}(1-t)^{\frac{3}{2}-\frac{\nu_{2}+1}{2}-1}}{\left(1-t\left(-\frac{\left(z-x-\mu_{2}\right)^{2}}{\nu_{2} \sigma_{2}^{2}}\right)\right)^{1 / 2}} d t \\
& =\frac{\Gamma\left(\frac{3}{2}\right)}{\Gamma\left(\frac{\nu_{2}+1}{2}\right) \Gamma\left(\frac{3}{2}-\frac{\nu_{2}+1}{2}\right)} \int_{0}^{1} t^{\frac{\nu_{2}+1}{2}-1}(1-t)^{\frac{3}{2}-\frac{\nu_{2}+1}{2}-1}\left(1-t\left(-\frac{\left(z-x-\mu_{2}\right)^{2}}{\nu_{2} \sigma_{2}^{2}}\right)\right)^{-1 / 2} d t \\
& \left.=\frac{\Gamma\left(\frac{3}{2}\right)}{\Gamma\left(\frac{\nu_{2}+1}{2}\right) \Gamma\left(\frac{3}{2}-\frac{\nu_{2}+1}{2}\right)} \int_{0}^{1} t^{\left(\frac{\nu_{2}+1}{2}\right)-1}(1-t)^{\left(\frac{3}{2}-\frac{\nu_{2}+1}{2}\right)-1}(1-t \underbrace{\left(-\frac{\left(z-x-\mu_{2}\right)^{2}}{\nu_{2} \sigma_{2}^{2}}\right)})\right)^{-1 / 2} d t(t)
\end{aligned}
$$

Pode-se Observar que (3) tem forma de uma distribuição beta,

$$
\begin{aligned}
& f(t)=\frac{\Gamma(a+b)}{\Gamma(a) \Gamma(b)} t^{a-1}(1-t)^{b-1}, 0<t<1 \\
& f(t)=\int_{0}^{1} \frac{\Gamma(a+b)}{\Gamma(a) \Gamma(b)} t^{a-1}(1-t)^{b-1} d t=1
\end{aligned}
$$

más para isso temos que fazer uma mudança de variável para a base $(1-t z)$ em nosso caso, $z$ é uma constante, já que não depende de $t$. 


\title{
Referências Bibliográficas
}

\author{
Alencar (2013) C. M. C.; Morettin P. A. Alencar, Airlane P.; Toloi. State space Markov switching \\ models using wavelets. Studies in Nonlinear Dynamics Econometrics, v. 0, p. 1-18, 2013. Citado \\ na pág. 67
}

Bollerslev (1986) T. Bollerslev. Generalized Autoregressive Conditional Heteroskedasticity. Journal of Econometrics, 31, 307-327. Citado na pág. 11, 14

Correa (2004) O. R. Correa, D. S. A. ; Hillbrecht. Ciclos Internacionais de Négocios: Uma Análise de Mudança de Regime Markoviano para Brasil, Argentina e Estados Unidos. Banco Central do Brasil. Citado na pág. 67

Dueker (1997) M. J. Dueker. Markov Switching in GARCH Processes and Reverting Stock Market Volatility. Journal of Business and Economic Statistics. 15(1), 26-34., first ed. Citado na pág. 1, 33

Engle (1987) Lilien D. M. Robins R. P. Engle, R. F. Estimating Time Varying Risk Premia in the Term-Structure: the ARCH-M Model. Econometrica, 55(2), 391-407. Citado na pág. 21

Engle (1989) R. F. Engle. Autoregressive Conditional Heteroskedasticity with Estimates of the Variance Of United Kingdom Inflation. Econometrica, 57(4), 987-1007. Citado na pág. 11, 13

Fama (1965) E. F. Fama. The behavior of stock market prices. Journal of Business, 38, 34-105. Citado na pág. 17

Fuller (1996) W. A. Fuller. Introduction to Statistical Time Series. John Wiley and Sons, New York, second ed. Citado na pág. 3

Gray (1996a) S.F. Gray. Modeling the conditional distribution of interest rates as a RegimeSwitching process. Journal of Financial Economics,42,27-62. Citado na pág. 27

Hamilton (1989) J. D. Hamilton. A New Approach to the Economic Analysis with of NonStationary Time Series and the Bussines Cycle. Econometrica, 57: 357-380. Citado na pág. iii, 1, 26,35

Hamilton e Susmel (1994) J. D. Hamilton e R. Susmel. Autoregressive Conditional Heterocedasticity and Changes in Regime. Journal of Econometrics. 64(1-2),307-333. Citado na pág. 3, 8

Hansen (1994) B. E. Hansen. Autoregressive Conditional Density Estimation. Journal of Applied Econometrics. Citado na pág. 33

Hayashi (2000) F. Hayashi. Econometrics. Princeton University Press, New Jersey. Citado na pág. 8

He (1999a) Terasvirta T. He, C. Properties of Moments of a Family of GARCH Processes. Journal of Econometrics, 92, 173-192. Citado na pág. 15

He (1999b) Terasvirta T. He, C. Fourth Moment Structure of the GARCH(p,q) Process. Econometric Theory, 15, 824-846. Citado na pág. 15 
Jarque (1980) Anil K. Jarque, Carlos M.; Bera. Efficient tests for normality, homoscedasticity and serial independence of regression residuals. Economics Letters 6 (3): 255259. Citado na pág. 18

Klaassen (2002) F. Klaassen. Improving GARCH Volatility Forecasts. Empirical Economics, 27(2), 363-394. Citado na pág. 1, 26

Mandelbrot (1963) B. B. Mandelbrot. The variation of certain speculative prices. Journal of Business, 36, 394-419. Citado na pág. 17

Marcucci (2005) Juri. Marcucci. Forecasting Stock Market Volatility with Regime-Switching GARCH Models. Studies in Nonlinear Dynamics and Econometrics, Volume 9, Issue 4, Article 6. Citado na pág. iii, 16, 17

Mittnik (2000) Rachev S. T. Mittnik, S. Stable Paretian Models in Finance. Wiley, New York. Citado na pág. 17

Mittnik (1998) Rachev S. T. Paolella M. S. Mittnik, S. Stable Paretian Models in Finance: Some empirical and theorical aspect, in R. J. Adler, R. E. Feldman and M. S. Taqqu. A Practial Guide to Heavy Tails: Statistical Techniques and Applications, 79-110, Birkhauser, Boston. Citado na pág. 17

Morettin (2006) Toloi C. M. C. Morettin, P.A. Análise de Séries Temporais. Edgar Blucher, São Paulo. Citado na pág. 3, 4, 9

Nelson (1991) D. B. Nelson. Condicional Heteroscedasticity in Asset Returns: A New Approach. Econometrica, 59(2),347-370. Citado na pág. 11

Peng. L. (2003) Q. Peng. L., Yao. Least absolute deviation estimation for ARCH and GARCH models. Biometrika, 90 (4), 967-975. Citado na pág. 17, 18

Shapiro (1965) M. B. Shapiro, S. S.; Wilk. An analysis of variance test for normality (complete samples). Biometrika 52 (3-4): 591611. Citado na pág. 18

White (1984) H. White. Asymptotic Theory for Econometricians. Academic Press, San Diego. Citado na pág. 8

Zivot (2003) Wang J. Zivot, E. Modelling Financial Time Séries with S-PLUS. Springer, New York. Citado na pág. 7, 31 